Supporting information for

\title{
Hydrogenation of Aldehydes Catalyzed by an Available Ruthenium Complex
}

Xuefeng Tan, Guozhen Wang, Ziyue Zhu, Conghui Ren, Jinping Zhou, Hui Lv, Xiao-yong Zhang,

Lung Wa Chung,* Lina Zhang* and Xumu Zhang* 


\section{Table of Contents}

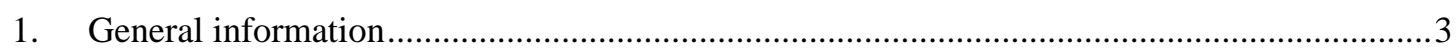

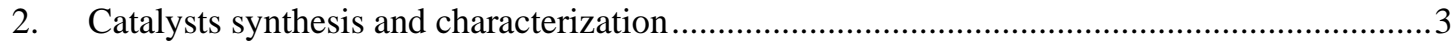

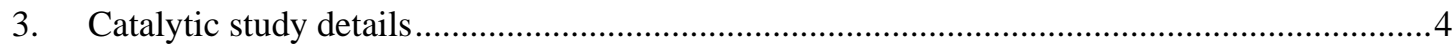

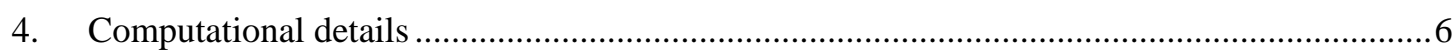

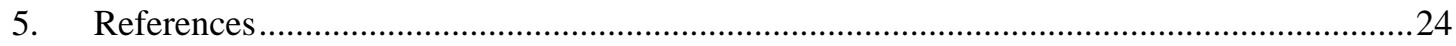

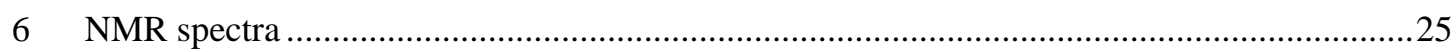

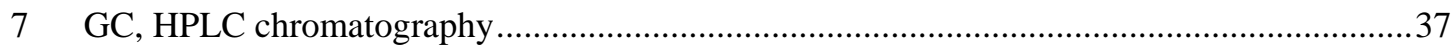




\section{General information}

Unless mentioned otherwise, all experiments were carried out under an atmosphere of argon or using standard Schlenk techniques. Solvents were dried with standard procedures and degassed with $\mathrm{N}_{2} .{ }^{1} \mathrm{H},{ }^{13} \mathrm{C},{ }^{31} \mathrm{P}$ NMR spectrum were recorded on NMR spectra were recorded on Bruker ADVANCE III (400 MHz) spectrometers for ${ }^{1} \mathrm{H},{ }^{13} \mathrm{C}$, ${ }^{31} \mathrm{P}$ NMR. With $\mathrm{CDCl}_{3}$ or $\mathrm{CD}_{2} \mathrm{Cl}_{2}$ as the solvent and tetramethylsilane as the internal standard. Chemical shifts were reported upfield to TMS (0.00 ppm) for ${ }^{1} \mathrm{H}$ NMR and relative to $\mathrm{CDCl}_{3}(77.0 \mathrm{ppm})$ for ${ }^{13} \mathrm{C}$ NMR. Column Chromatography was performed with silica gel Merck 60 (300-400 mesh). All the substrates were distilled once (benzaldehyde twice).

\section{Catalysts synthesis and characterization}

Ru1 were synthesized as follow:

Under a nitrogen atmosphere, $\left[\mathrm{RuCl}_{2} \text { (benene) }\right]_{2}(125 \mathrm{mg}, 0.25 \mathrm{mmol})$ and 1,3-bis(diphenylphosphino) propane (DPPP) (206 mg, $0.5 \mathrm{mmol}$ ) were dissolved in $5 \mathrm{~mL} \mathrm{~N}, \mathrm{~N}$-dimethylformamide. The mixture was heated at $110^{\circ} \mathrm{C}$ for $2 \mathrm{~h}$. Upon cooled to room temperature, a solution of sodium glycinate $(145 \mathrm{mg}, 1.5 \mathrm{mmol})$ in methanol $(10 \mathrm{~mL})$ was added into the mixture. After stirring at room temperature overnight, the mixture was poured into $50 \mathrm{~mL}$ water. The mixture was extracted with dichloromethane $\left(20 \mathrm{~mL}\right.$ by three times) and dried with $\mathrm{Na}_{2} \mathrm{SO}_{4}$. After concentrated, the crude product was purified by chromatography to obtain a yellow solid (192 mg, yield 60\%). ${ }^{1} \mathrm{H}$ NMR(400 MHz, CD $\left.3 \mathrm{OD}\right): \delta=7.39-7.54(\mathrm{~m}, 4 \mathrm{H})$, 7.12-7.41 (m, 16H), 4.41-4.57 (m, 2H), 3.14-3.25 (m, 2H), 2.69-2.79 (m, 2H), 2.57-2.69 (br, 4H), 2.44-2.57 (m, 2H), 1.85-2.08 (m, 2H); ${ }^{13} \mathrm{C}$ NMR (101 MHz, $\left.\mathrm{CDCl}_{3}\right) \delta=186.0,137.3,137.1,136.9,136.2,136.1,135.9,133.6,133.2,130.9,129.9$, 129.9, 129.8, 129.6, 129.6, 129.5, 45.1, 27.3, 27.1, 26.9, 19.7; ${ }^{31} \mathrm{P}$ NMR (161.7 MHz, $\mathrm{CD}_{3} \mathrm{OD}$ ): $\delta=43.16$ (s). Single crystal of Ru1 was obtained from the crystallization from dichloromethane and ethyl ether.

CCDC 1063360 contains the supplementary crystallographic data for this paper.

These data can be obtained free of charge via 
www.ccdc.cam.ac.uk/conts/retrieving.htmL (or from the Cambridge Crystallographic Data Centre, 12, Union Road, Cambridge CB21EZ, UK; fax: (+44) 1223-336-033; or deposit@ @cdc.cam.ac.uk).

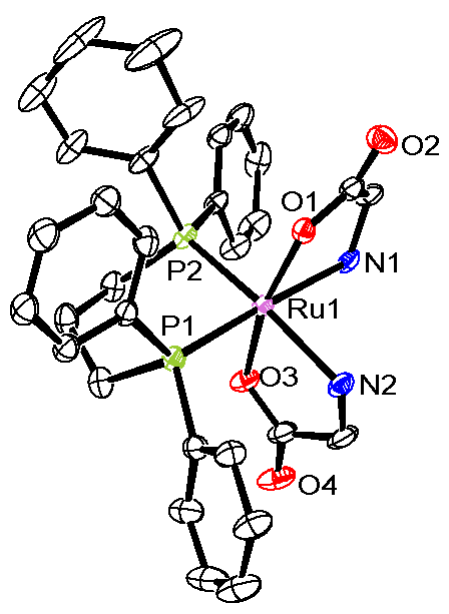

Figure S1. ORTEP diagrams for Ru3. Selected bond lengths $(\AA)$ and angles (deg): $\mathrm{N}(1)-\mathrm{Ru}(1)$ 2.179(5)， $\mathrm{N}(2)-\mathrm{Ru}(1) \quad 2.182(5), \quad \mathrm{O}(1)-\mathrm{Ru}(1)$ 2.106(4), $\mathrm{O}(3)-\mathrm{Ru}(1)$ 2.099(5), $\quad \mathrm{P}(1)-\mathrm{Ru}(1) \quad 2.2642(16), \quad \mathrm{P}(2)-\mathrm{Ru}(1) \quad 2.2620(15), \quad \mathrm{O}(1)-\mathrm{Ru}(1)-\mathrm{N}(1)$ 78.74(18), O(3)-Ru(1)-N(2) 80.6(2), N(1)-Ru(1)-N(2) 84.69(18), P(1)-Ru(1)-P(2) 89.97(6).

\section{Catalytic study details}

In an argon glovebox, a $5 \mathrm{~mL}$ vial equipped with a magnetic stirring bar, was added the required amount of catalyst (dissolved in relative substrates) and substrates. The mixture was transferred to a stain-less autoclave and then was purged by three cycles of pressurization/venting with $\mathrm{H}_{2}(10 \mathrm{~atm})$ then pressurized with $\mathrm{H}_{2}(50 \mathrm{~atm})$ and disconnected from the $\mathrm{H}_{2}$ source. Then the autoclave was placed in an oil bath preheated to the desired temperature. After the desired reaction time, the autoclave was cooled in an ice bath, and the pressure was released slowly.

Data in table 1 and table 2 were obtained as the following procedure:

The resulting mixture was added internal standard (except for p-methoxybenzaldehyde and m-methoxybenzaldehyde using mesitylene as internal, the other substrates were all using n-tridecane as internal), got one drop for GC 
analysis and the rest samples were purified by column chromatography (washed with hexane/ethyl acetate $=5: 1)$. GC column (SPBTM-5, FUSED SILICA Capillary Column, $30 \mathrm{~m} \times 0.25 \mathrm{~mm} \times 0.25 \mu \mathrm{m}$, film thickness).

Data in table 3 were obtained as the following procedure:

For entries 1-5, the reaction mixtures were concentrated under reduced pressure, dissolved in water and filtered, the filtrate can be used for HPLC analyse directly. For entries 6-8, the acid was neutralized using $\mathrm{CaCO}_{3}$. Filter and remove of the $i$-PrOH, the products were analysed by high-performance liquid chromatography (HPLC; Agilent 1100 Series) equipped with a refractive index detector and a Hi-Plex Ca column $\left(300 \times 7.7 \mathrm{~mm}\right.$, mobile phase: water $\left.1.0 \mathrm{~mL} \mathrm{~min}^{-1}, 80^{\circ} \mathrm{C}\right)$. The glucose conversion was determined by HPLC before and after the reaction. The yield of each product was calculated as follows: yield $(\%)=100 \% \times($ mols of carbon in each product)/(mols of carbon in charged glucose). ${ }^{1}$

Table S1, Effect of hydrogenation pressure ${ }^{a}$

\begin{tabular}{lcc}
\hline & \multicolumn{1}{c}{$\begin{array}{c}\mathrm{H}_{2}(50 \mathrm{bar}) \\
100^{\circ} \mathrm{C}, 24 \mathrm{~h}\end{array}$} \\
\hline Entry & $\mathrm{H}_{2}(\mathrm{~atm})$ & 51 \\
\hline 1 & 10 & 67 \\
2 & 30 & $>99$ \\
3 & 50 & $>99$ \\
4 & 70 & Yield $(\%)^{b}$ \\
\hline
\end{tabular}

${ }^{a}$ Reaction conditions: benzaldehyde (10 mmol), Ru1 (0.002 $\mathrm{mol} \%), i-\operatorname{PrOH}(2 \mathrm{~mL}), 100^{\circ} \mathrm{C}, 24 \mathrm{~h} .{ }^{b}$ Yield was determined by $\mathrm{GC}$ analysis, $\mathrm{n}$-tridecane was used as internal standard.

Table S2. Details data for the hydrogenation of Substrate S1-S11 ${ }^{a}$

$\begin{array}{ccccc}\text { entry } & \text { substrate } & \text { S/C } & \begin{array}{c}\text { isolated yield }(\%) \\ (\mathrm{g})^{b}\end{array} & \begin{array}{c}\text { physical } \\ \text { status/color }\end{array} \\ 1^{d} & \mathrm{~S} 1 & 300000 & 91(4.85) & 1 / \text { colorless } \\ 2^{d} & \mathrm{~S} 1 & 400000 & 81(4.3) & \\ 3 & \mathrm{~S} 2 & 150000 & 94(1.28) & 1 / \text { colorless } \\ 4 & \mathrm{~S} 2 & 200000 & 86(1.17) & \\ 5 & \mathrm{~S} 3 & 200000 & 96(1.31) & \text { s/colorless } \\ 6 & \mathrm{~S} 3 & 250000 & 82(1.12) & \end{array}$




$\begin{array}{lcccc}7 & \text { S4 } & 20000 & 94(1.17) & 1 / \text { colorless } \\ 8 & \text { S4 } & 50000 & 67(0.83) & \\ 9 & \text { S5 } & 50000 & 92(1.44) & 1 / \text { colorless } \\ 10 & \text { S5 } & 100000 & 71(1.11) & \\ 11 & \text { S6 } & 100000 & 98(0.94) & 1 / \text { colorless } \\ 12 & \text { S6 } & 150000 & 79(0.76) & \\ 13 & \text { S7 } & 100000 & 92(0.92) & 1 / \text { colorless } \\ 14 & \text { S7 } & 150000 & 77(0.77) & \\ 15 & \text { S8 } & 100000 & 94(1.47) & 1 / \text { colorless } \\ 16 & \text { S8 } & 150000 & 86(1.34) & \\ 17 & \text { S9 } & 100000 & 92(1.03) & 1 / \text { colorless } \\ 18 & \text { S9 } & 150000 & 77(0.86) & \\ 19 & \text { S10 } & 100000 & 96(1.48) & 1 / \text { colorless } \\ 20 & \text { S10 } & 150000 & 90(1.38) & \\ 21^{e} & \text { S1 }+ \text { S11 } & 100000 & 95(1.01): 0(0) & \end{array}$

${ }^{a}$ Reaction conditions: aldehyde $(10 \mathrm{mmol}), \mathrm{H}_{2}(50 \mathrm{~atm}), 100{ }^{\circ} \mathrm{C}, 24 \mathrm{~h} .{ }^{b}$ isolated yield, data in parenthese was the amount of isolated product. ${ }^{c} 1$ means liquid, s means solid. ${ }^{d}$ Substrate (50 mmol). ${ }^{e} \mathrm{~S} 1: \mathrm{S} 11$ (5 mmol:5 mmol), Ru1 $\left(1 \times 10^{-4} \mathrm{mmol}\right)$.

\section{Computational details}

We performed density functional theory (DFT) calculation for two Ru isomeric, models $\mathbf{1}$ and $\mathbf{2}$ to construct their free-energy reaction profiles for the hydrogenation of acetaldehy complexes de as shown in Scheme S1. The model $\mathbf{1}$ was adopted from the X-ray crystal structure of Ru1, whereas the model $\mathbf{2}$ was modified from the model 1 by dissociation of one amine part. Hybrid B3LYP method ${ }^{2}$ (combined with 6-31G(d) basis set for light atoms and SDD basis set plus effective core potentials (ECPs) ${ }^{3}$ for $\mathrm{Ru}$ ) was used to optimize all structures, and Grimme's dispersion (D3) corrections ${ }^{4}$ were also applied in the optimization calculations. The reactants, intermediates and products were verified to have no imaginary frequency by harmonic vibrational frequency calculation, while the transition states were also verified to present an imaginary frequency relating to the reaction coordinates. Single-point energy calculations in solution were performed on these gas-phase optimized structures by 
the M06 method ${ }^{5}$ (with the same basis sets) and an implicit solvent model SMD 6 (2-propanol as the solvent). All the computations were conducted using the Gaussian 09 package. $^{7}$

Various attack modes of hydrogen and the aldehyde towards the catalyst Ru3 have been considered in the rate-determining transition states (i.e., $\mathrm{TS}_{8-10}$ ). These attack modes (a-f) are displayed in Figure S2. Their solvation free energies (kcal/mol) by the M06-SMD///B3LYP-D3 and B3LYP-D3-SMD (in parentheses) methods are provided as well.

Our calculations reveal that modes a-I and d-I are much more energetically favorable than the other modes. The mode $\mathbf{~ d - I}$ is $2.2 \mathrm{kcal} / \mathrm{mol}$ lower in energy than its stereoisomeric mode a-I by the M06-SMD///B3LYP-D3 method, but $0.7 \mathrm{kcal} / \mathrm{mol}$ higher by the B3LYP-D3-SMD method. These suggest both modes with the same path can be competitive. In addition, our additional calculations showed that the hydrogenation barrier of acetone (at least $33.1 \mathrm{kcal} / \mathrm{mol}$ ) is higher than that of aldehyde (at least $25.9 \mathrm{kcal} / \mathrm{mol}$ ) at least $7.2 \mathrm{kcal} / \mathrm{mol}$ in the solution (see Fig. S4), which nicely explains the chemoselectivity of this hydrogenation. 
Scheme S1 Two possible models for reaction cycles of the aldehyde hydrogenation catalyzed by the model 1 (Ru1).

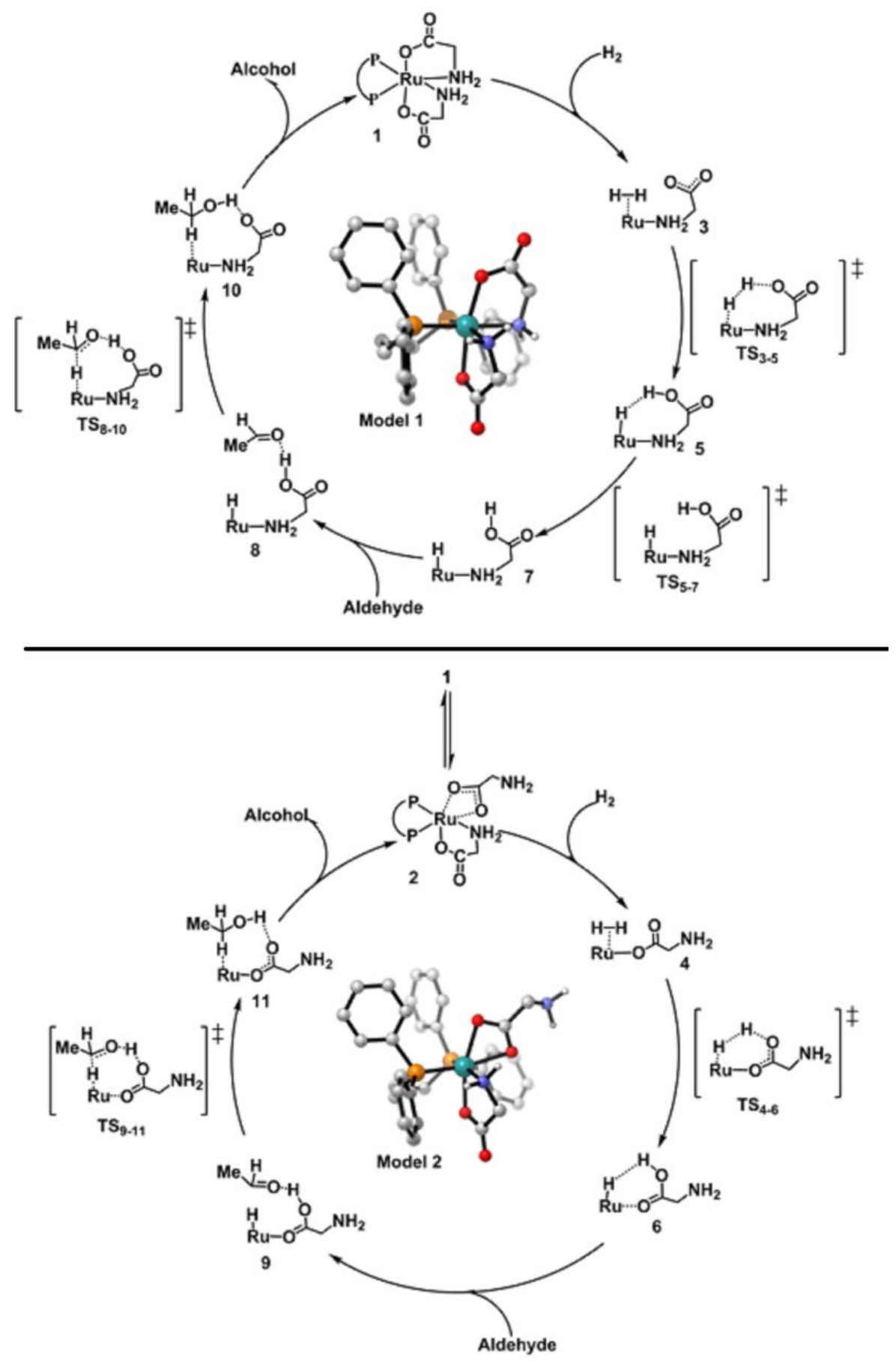




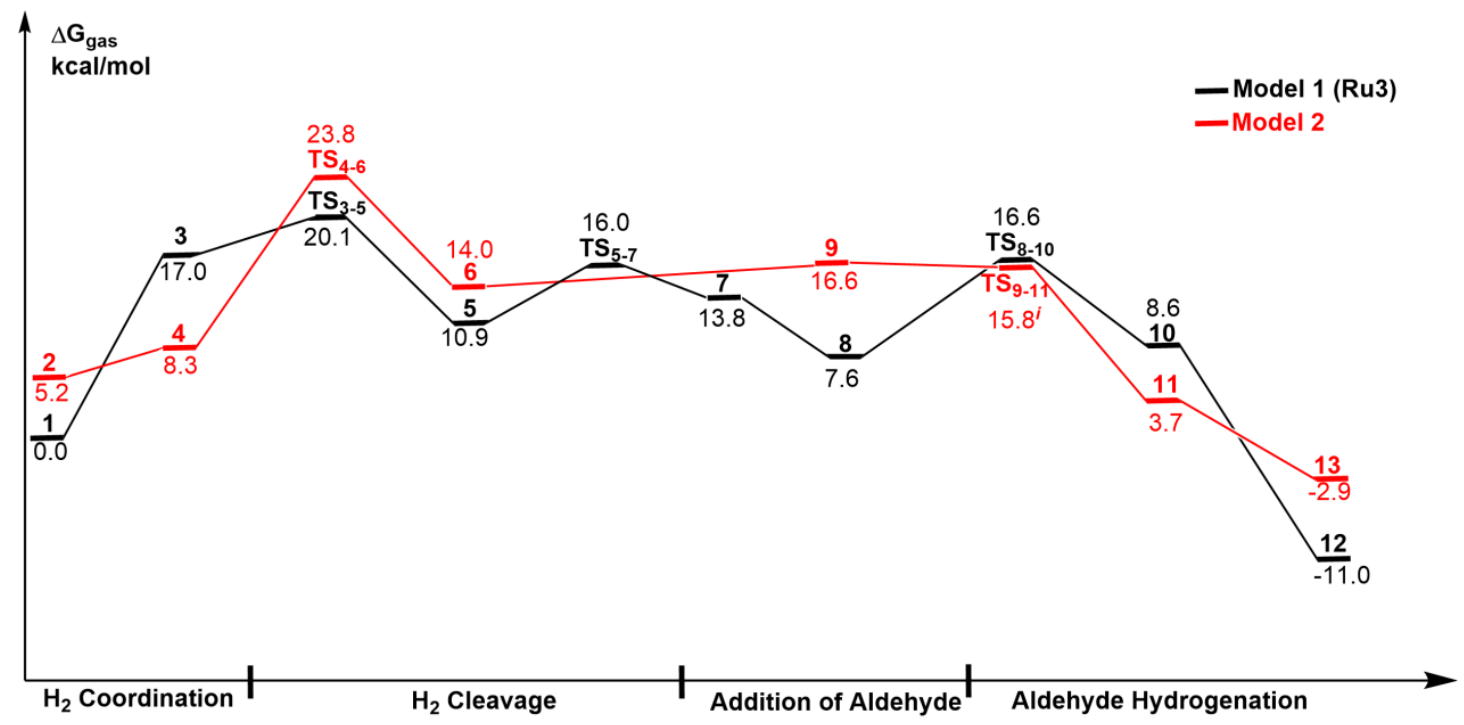

Figure S2 Free energy ( $\mathrm{kcal} / \mathrm{mol})$ profile of the aldehyde hydrogenation catalyzed by the model 1 (Ru3) and model 2 in gas phase by the M06//B3LYP-D3 method. All energies are referred to model $\mathbf{1}$. The nomenclature is referred to Scheme $\mathrm{S} 1$.

The intermediate 9 is calculated to be $0.8 \mathrm{kcal} / \mathrm{mol}$ higher in free energy than TS9-11 in gas phase by the M06//B3LYP-D3 method. It is noted that the relative electronic energy of $\mathbf{9}$ is only $0.02 \mathrm{kcal} / \mathrm{mol}$ lower than that of $\mathbf{T S}_{9-11}$ by the B3LYP-D3 method. The almost degenerate electronic energy of 9 and TS9-11 could bring this unphysical ordering in free energy. 


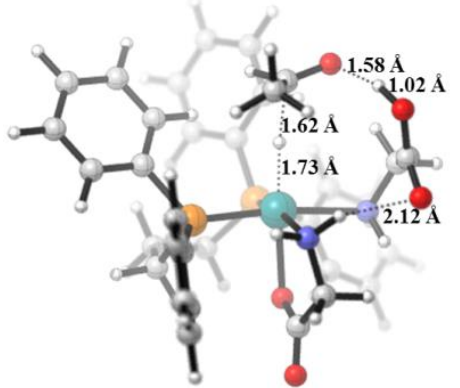

a-I $\left(\mathrm{TS}_{8-10}\right) \stackrel{26.0}{(25.9)}$

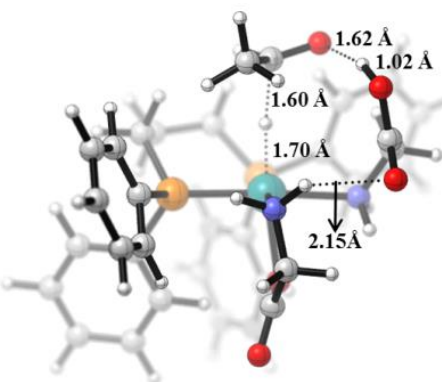

23.8

d-I

(26.6)

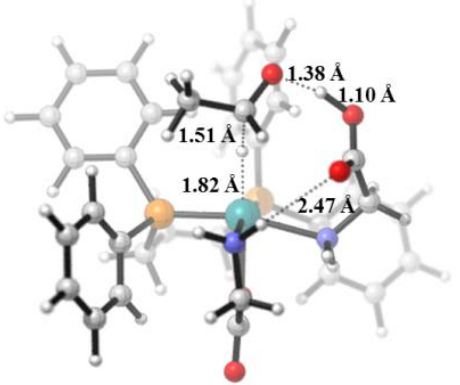

31.7

b-I

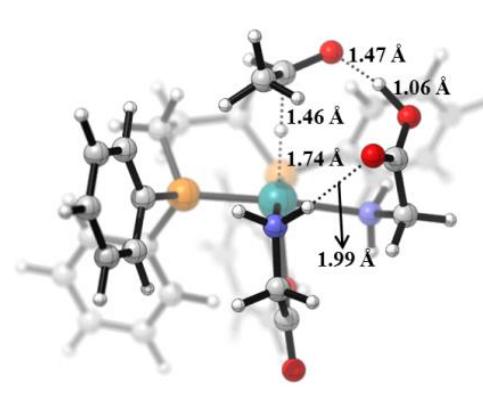

27.3

e-I

(29.1)

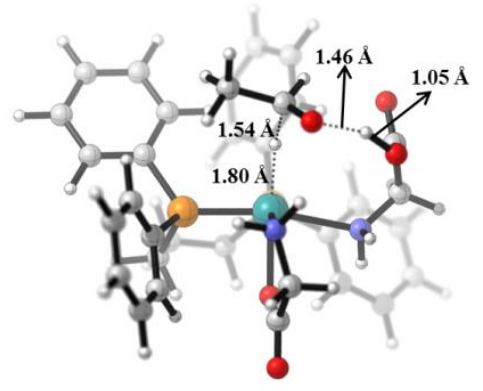

32.3

(31.4)

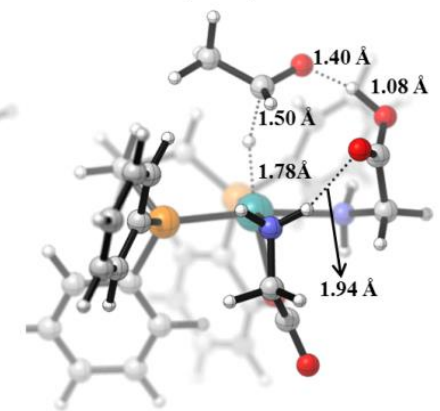

29.3

f-I (31.1)

Figure S3 Six isomeric transition state structures (a-f) for the rate-determining hydrogenation step of the aldehyde (I) catalyzed by Ru3 and their corresponding solvation free energies (kcal/mol) using the M06-SMD///B3LYP-D3 method. Values in parentheses are the solvation free energy by the B3LYP-SMD method. 


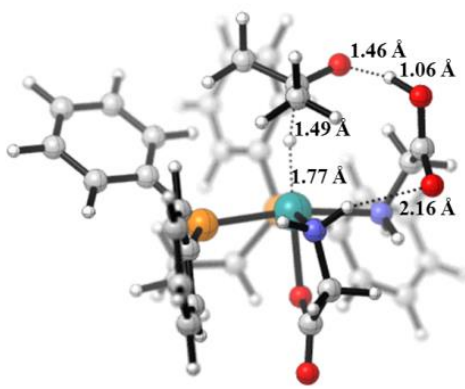

a-II $\begin{gathered}32.0 \\ (34.7)\end{gathered}$

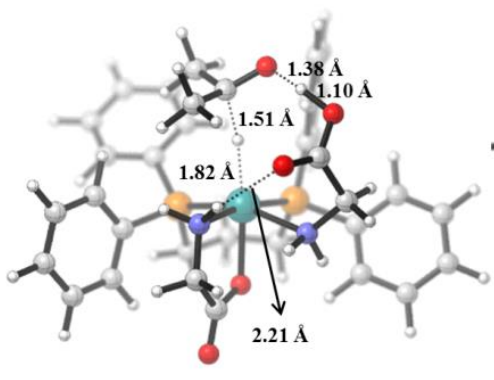

b-II $\quad 40.8$

(40.9)

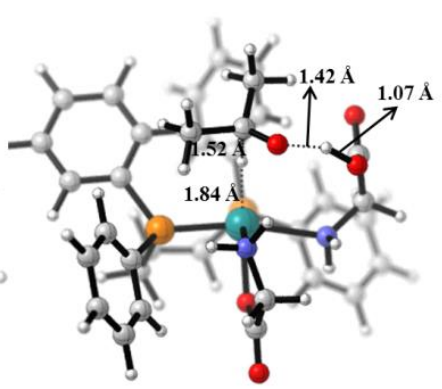

c-II 37.3 (36.5)

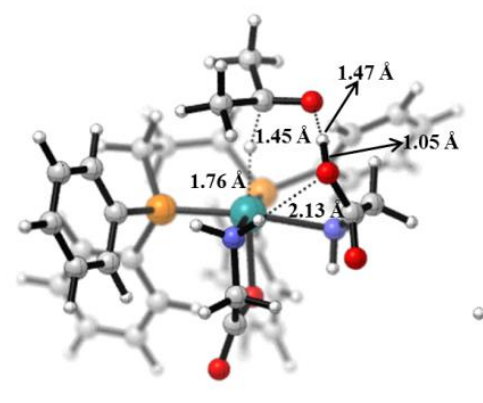

d-II

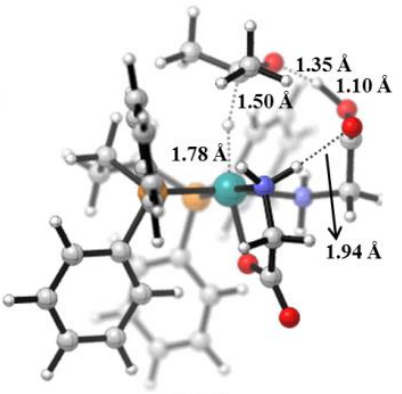

33.5

e-II (35.5)

Figure S4 Five isomeric transition state structures (a-e) located for the rate-determining hydrogenation step of the acetone (II) catalyzed by Ru3 and their corresponding solvation free energies $(\mathrm{kcal} / \mathrm{mol})$ using M06-SMD///B3LYP-D3 and B3LYP-SMD (in parentheses) methods. 
Table S3 Absolute electronic energy $\left(E_{\text {gas }}\right)$, Gibbs free energy $\left(G_{\text {gas }}\right)$ and thermal correction to Gibbs Free Energy $\left(\Delta \mathbf{G}_{\mathrm{GC}}\right)$ using B3LYP-D3 method, as well as the solvation electronic energy $\left(E_{\text {smd }}\right)$ using M06-SMD///B3LYP-D3 method for intermediates and transition states structures.

\begin{tabular}{|c|c|c|c|c|}
\hline & $\mathrm{E}_{\mathrm{gas}}(\mathrm{au})$ & $\mathrm{G}_{\text {gas }}(\mathrm{au})$ & $\Delta \mathrm{G}_{\mathrm{GC}}(\mathrm{au})$ & $\mathrm{E}_{\text {smd }}(\mathrm{au})$ \\
\hline $\mathbf{H}_{2}$ & -1.17548241168 & -1.176825 & -0.001342 & -1.16628802324 \\
\hline Aldehyde & -153.832051964 & -153.801218 & 0.030834 & -153.737319883 \\
\hline Acetone & -193.160047250 & -193.103849 & 0.056198 & -193.032738047 \\
\hline 1 & -2390.09819159 & -2389.566643 & 0.531549 & -2388.85270900 \\
\hline 3 & -2391.26279269 & -2390.713602 & 0.549191 & -2390.01643899 \\
\hline TS $_{3-5}$ & -2391.25649683 & -2390.710539 & 0.545958 & -2390.00096448 \\
\hline 5 & -2391.27014913 & -2390.721574 & 0.548575 & -2390.01665844 \\
\hline TS $_{5-7}$ & -2391.25949769 & -2390.712669 & 0.546829 & -2390.00211437 \\
\hline 7 & -2391.26117573 & -2390.713527 & 0.547649 & -2390.01333569 \\
\hline 8 & -2545.12641917 & -2544.524454 & 0.601965 & -2543.77340374 \\
\hline $\begin{array}{c}\text { TS }_{8-10} \\
(\mathbf{a}-\mathrm{I})\end{array}$ & -2545.12637684 & -2544.517570 & 0.608807 & -2543.76267830 \\
\hline 10 & -2545.14299172 & -2544.529630 & 0.613362 & -2543.78936479 \\
\hline 12 & -2545.16818922 & -2544.560185 & 0.608004 & -2543.81191830 \\
\hline 2 & -2390.08390691 & -2389.555389 & 0.528517 & -2388.83302225 \\
\hline 4 & -2391.26990039 & -2390.725659 & 0.544241 & -2390.01073563 \\
\hline $\mathrm{TS}_{4-6}$ & -2391.25765817 & -2390.706779 & 0.550879 & -2389.99398069 \\
\hline 6 & -2391.26165107 & -2390.715720 & 0.545931 & -2390.00560641 \\
\hline 9 & -2545.11360877 & -2544.514303 & 0.599306 & -2543.75639708 \\
\hline TS9-11 $_{9}$ & -2545.11357555 & -2544.515204 & 0.598372 & -2543.75442908 \\
\hline 11 & -2545.14635793 & -2544.537127 & 0.609231 & -2543.78804125 \\
\hline 13 & -2545.14796416 & -2544.544096 & 0.603868 & -2543.79167407 \\
\hline TS(d-II) & -2584.44044711 & -2583.808147 & 0.632300 & -2583.0483977 \\
\hline
\end{tabular}

Cartesian Coordinates of Key $\mathrm{H}, 0,3.4659090095,17.5328040939,21.7850642565$ Structures

\section{Catalyst Ru3 (Model 1)}

C,0,7.2115533691,19.4202710723,14.738977953 C, $0,7.2799378771,17.9573891264,14.2500131781$ $\mathrm{H}, 0,6.7033094863,17.8923677013,13.3217433116$ $\mathrm{H}, 0,8.3177162969,17.6950338675,14.0234296068$ C,0,4.6868988781,15.9070753335,18.0892592329 C,0,6.0069754672,16.6013240567,18.5185842749 $\mathrm{H}, 0,6.0799651188,16.564963989,19.6085233378$ H,0,6.8295123129,16.0002797656,18.1140180532 C,0,3.4999955255,19.4685013128,18.9738204508 C, $0,3.3148159465,18.346323672,19.8028021779$ $\mathrm{H}, 0,2.9590648066,17.4068582701,19.3930134213$ C,0,3.6141195067,18.4121919328,21.1643208501
C,0,4.1078299386,19.5946291797,21.7204680184 H,0,4.3435289472,19.6434895437,22.7800827608 C,0,4.3007751603,20.7118234365,20.9058102296 H,0,4.6842477085,21.6365183862,21.3290552424 C,0,4.0011161765,20.6503755008,19.5427224148 $\mathrm{H}, 0,4.1561757887,21.5262342284,18.9201067662$ C,0,2.9490429174,21.0231344856,16.5942941194 C,0,4.0357379102,21.804335904,16.1665237625 H,0,5.0288119986,21.3664522689,16.1198507829 C,0,3.8342837354,23.1241093625,15.7584216762 $\mathrm{H}, 0,4.6837588654,23.7158205674,15.4276174779$ C, $0,2.5512654753,23.6734604515,15.7573100644$ H,0,2.3949290567,24.6974627563,15.4281362466 C,0,1.4661264969,22.9016191758,16.1778124355 H,0,0.4640928906,23.3226637424,16.1785525689 
C,0,1.6638574178,21.5872414628,16.6012286553 $\mathrm{H}, 0,0.8084296765,21.0082364123,16.9345433404$ C,0,4.2080278278,16.4085379184,13.2491564507 C,0,4.3476920611,15.1487191872,13.863977799 H,0,4.0764898226,15.0276804478,14.9108447228 C,0,4.8579324351,14.0662333802,13.1459810579 $\mathrm{H}, 0,4.9556586496,13.0985806123,13.6313224009$ C,0,5.2447783682,14.2258247393,11.8118938347 H,0,5.6457675436,13.3829869369,11.2554730169 C,0,5.115166495,15.4726008615,11.1989509327 H,0,5.4160264511,15.6048904466,10.1629669962 C,0,4.5997817376,16.5589358844,11.9117654645 $\mathrm{H}, 0,4.5018820083,17.5236420442,11.4228220238$ C,0,4.0616014735,20.3338373944,13.1724210528 H,0,4.8497576498,20.4125689955,13.9115061367 C,0,3.8115614985,21.394346312,12.2987382176 $\mathrm{H}, 0,4.4151896523,22.2948838657,12.3666473729$ C,0,2.7888890901,21.3000224297,11.3554929492 $\mathrm{H}, 0,2.5915660477,22.1273089571,10.6787331273$ C,0,2.0159206282,20.1371947267,11.2806001376 $\mathrm{H}, 0,1.2200507571,20.0572293849,10.5446291628$ C, $0,2.2628858345,19.0775309873,12.1517128142$ H, $0,1.6603221632,18.1768346135,12.0739726105$ C,0,3.2860268485,19.1677713078,13.111454785 C, $0,1.9347740118,17.2171937768,14.7806884662$ $\mathrm{H}, 0,1.3880466447,16.9349924001,13.8748878232$ H,0,2.0960337826,16.3074093664,15.3685988664 C, $0,1.1207810221,18.2311542485,15.6032411246$ H,0,0.0909222528,17.8580359219,15.6725250511 H,0,1.0636076981,19.1904852321,15.0729939554 C,0,1.6412421,18.4346097757,17.0379768803 H,0,1.8100585565,17.4554819213,17.4971411488 H,0,0.9037886925,18.9648636516,17.6500278945 $\mathrm{N}, 0,6.6551744059,17.0231946883,15.2242793029$ $\mathrm{H}, 0,7.3491003185,16.7376793807,15.9154444068$ $\mathrm{H}, 0,6.3470561371,16.1682700842,14.7599521952$ $\mathrm{N}, 0,6.1330514855,17.9889692117,17.9963268052$ $\mathrm{H}, 0,7.1065218851,18.2619611272,17.8553752504$ H,0,5.7566885334,18.64743055,18.6773334092 O,0,6.2096482584,19.6958074621,15.5267779071 O,0,8.0432251564,20.2222560267,14.3349250041 O,0,4.122854203,16.3513598835,17.0031536376 $\mathrm{O}, 0,4.281656519,14.9726481238,18.769737617$ P,0,3.2820480288,19.2986011059,17.141928144
$\mathrm{P}, 0,3.6344598897,17.8132767458,14.3068028199$ $\mathrm{Ru}, 0,4.9665016125,18.1001668236,16.1487125562$

\section{Model 2}

C,0,7.1163133364,18.3684683773,15.0441067067 C,0,8.2889791925,18.5641001711,14.1038575737 H,0,8.5937184311,19.6140428569,14.1184975339 H,0,9.1288719746,17.9411780603,14.4534164909 C,0,4.5826144059,15.7851456836,18.1280975449 C,0,5.9840773261,16.3522110115,18.4414021984 H,0,6.2260736172,16.1548771401,19.4896034966 H,0,6.698094838,15.8192735141,17.8055395458 C,0,3.4183536977,19.4652951729,19.0247853433 C,0,3.2741582686,18.3610282755,19.8850438897 $\mathrm{H}, 0,2.9781824352,17.3930727976,19.4928498863$ C,0,3.5339289226,18.4897145308,21.2502442343 H,0,3.4168253342,17.6264380888,21.8996394503 C,0,3.9462324535,19.7171324726,21.7754693516 $\mathrm{H}, 0,4.1492758774,19.815251306,22.8384744663$ C,0,4.1002569738,20.8156855288,20.9275342072 $\mathrm{H}, 0,4.4219674746,21.7733768085,21.328466366$ C,0,3.8413505284,20.691249722,19.5606098398 H,0,3.9622943393,21.5516940096,18.9089591594 C,0,2.8790052134,20.895021638,16.5467793679 C,0,3.75590581,21.4574746642,15.6092725938 H,0,4.6493242507,20.9172614181,15.3180368574 C,0,3.4719560702,22.6988095763,15.0360037886 H,0,4.1475246432,23.1093317944,14.2914369503 C, $0,2.3181531048,23.3920250646,15.4009613649$ H,0,2.0941086053,24.3550321641,14.9493923551 C, $0,1.4471627687,22.8466139024,16.349225427$ H,0,0.5501706984,23.386707477,16.6410420307 C,0,1.7253848104,21.6051895505,16.9188929154 $\mathrm{H}, 0,1.0435083023,21.1918293046,17.6575385032$ C,0,4.28898133,16.3872793489,13.3429434999 C,0,4.5335454826,15.1719965057,14.0064493089 $\mathrm{H}, 0,4.3333313178,15.0889211304,15.0715353691$ C,0,5.0536374633,14.0833375176,13.308996153 H,0,5.2366465532,13.1488585852,13.832865237 C,0,5.3536349382,14.1967793116,11.9486096262 H,0,5.7679921701,13.3492295107,11.4086304733 C,0,5.1212918479,15.4031295684,11.2868548094 $\mathrm{H}, 0,5.3512199077,15.4991257513,10.228602345$ C, $0,4.5873718013,16.4940376868,11.9783769682$ 
$\mathrm{H}, 0,4.4138761232,17.4271482918,11.4526914785$ C,0,4.6533415728,19.8222995497,12.7204586024 H,0,5.6296114015,19.4607029567,13.0226780542 C,0,4.5734105136,20.9221834017,11.8677300787 H,0,5.4889703904,21.3987196108,11.5269979585 C,0,3.3305622847,21.4098397969,11.4565820604 $\mathrm{H}, 0,3.2705695125,22.2758907374,10.80246825$ C,0,2.1660700403,20.7713484355,11.8823485449 $\mathrm{H}, 0,1.1937928621,21.1344621339,11.5597530227$ C,0,2.2426338739,19.6592423749,12.7230466859 H,0,1.3220768986,19.1731289645,13.0253021926 C,0,3.4838302459,19.1815867794,13.1678624454 C,0,1.9238121926,17.239278588,14.7496258496 H,0,1.3964995335,17.0317139772,13.8113177337 H,0,2.0619126971,16.2838406247,15.2680440174 C,0,1.0902428924,18.1844626372,15.6406080127 $\mathrm{H}, 0,0.0738797038,17.7724423507,15.6906208716$ H,0,0.9952638182,19.1735036287,15.1779158664 C, $0,1.5973984577,18.3447794826,17.0847560752$ H, $0,1.7586078766,17.3586001876,17.5302892646$ $\mathrm{H}, 0,0.8534952905,18.8775356363,17.6872567785$ $\mathrm{N}, 0,7.7967327296,18.2266944654,12.7571703606$ $\mathrm{H}, 0,7.4848484655,17.2558504967,12.7504046904$ $\mathrm{H}, 0,8.5548577872,18.3020054393,12.081703896$ $\mathrm{N}, 0,6.079576239,17.7965750829,18.0980473103$ $\mathrm{H}, 0,7.0488231343,18.0685795558,17.9356004835$ H,0,5.7303119374,18.3671243393,18.8676812558 O,0,6.7768928749,17.1859658869,15.3611812469 O,0,6.4031985277,19.3571128148,15.417669009 $\mathrm{O}, 0,3.9670787434,16.3354236182,17.1154427094$ O,0,4.142795304,14.859357977,18.7971292834 $\mathrm{P}, 0,3.2364532611,19.2160050218,17.2056176363$ $\mathrm{P}, 0,3.657997986,17.7895665811,14.3632986563$ $\mathrm{Ru}, 0,4.9014349213,18.0214161457,16.2786369091$

TS 3-5

C,0,8.2088899371,18.749070459,15.7262609731 C,0,7.7384329243,17.8125539451,14.5936874216 $\mathrm{H}, 0,7.3551582961,18.3626071506,13.7311018437$ $\mathrm{H}, 0,8.5506136531,17.1461804228,14.2847402875$ C,0,4.7880420482,16.0638221096,18.0667475942 C,0,6.0262775612,16.8587727939,18.5420747832 $\mathrm{H}, 0,5.984882853,16.9401296333,19.6304775477$ H,0,6.9120295626,16.2608254083,18.2984663209
C,0,3.4792575472,19.4457423279,18.9693034076 C,0,3.278303814,18.353934869,19.8301604729 $\mathrm{H}, 0,2.8766752139,17.417536212,19.4574702244$ C,0,3.6334404123,18.4425540507,21.1775016861 H,0,3.4756319547,17.5860738382,21.8269195098 C,0,4.2002957973,19.6146841438,21.6812741981 $\mathrm{H}, 0,4.4817755111,19.6784332822,22.7287388114$ C,0,4.4117316537,20.7028877771,20.8311353026 $\mathrm{H}, 0,4.8564615227,21.6173635378,21.2141182931$ C,0,4.0554151361,20.6197544562,19.484243627 H,0,4.2243726668,21.4705090817,18.8297506299 C,0,2.6119841001,20.9479044126,16.668339245 C,0,3.1759341373,21.6257600648,15.5816692732 H,0,4.0092640224,21.1869981389,15.0489116094 C,0,2.6675826775,22.8609590497,15.1726058109 $\mathrm{H}, 0,3.1113087143,23.3629133112,14.3175328635$ C,0,1.5960097556,23.4363385008,15.8536764236 H,0,1.1992516282,24.3972465772,15.5368552446 C,0,1.034517063,22.7757060892,16.9512883883 H,0,0.2054308119,23.2239768046,17.4924125228 C,0,1.5377590826,21.5408879164,17.3548346127 $\mathrm{H}, 0,1.1023728484,21.0417069205,18.216599471$ C,0,4.128397034,16.4840976665,13.1605600496 C,0,4.3137262276,15.2308909707,13.7778199225 H,0,4.1325543493,15.1226815208,14.8456953671 C,0,4.7543221437,14.1372504117,13.0317714104 H,0,4.8919340343,13.1760147701,13.5196898218 C,0,5.0232782022,14.2786281593,11.667488868 H,0,5.3710052119,13.4270917699,11.0890430198 C,0,4.8465491634,15.5183564102,11.0520900889 H,0,5.0544005183,15.6350533114,9.9917220826 C,0,4.4012428993,16.6166974326,11.7926224057 $\mathrm{H}, 0,4.2649424857,17.5747226124,11.30069508$ C,0,4.4492313779,20.0344350258,12.6764411813 H,0,5.4305011375,19.7766443106,13.0643043141 C,0,4.3122812724,21.1317514574,11.8282275255 H,0,5.1903930331,21.7053424034,11.544380386 C,0,3.0480535989,21.5005407806,11.3597575246 $\mathrm{H}, 0,2.9373598037,22.364811428,10.7105455023$ C,0,1.9294956242,20.7537194998,11.7290372108 H,0,0.9432685017,21.0334525537,11.3686899371 C,0,2.0694854586,19.6440052588,12.5656644168 H,0,1.1848622296,19.0772498182,12.8349596543 C,0,3.3284986126,19.2777722654,13.0600873551 
C,0,1.8864175133,17.2854042421,14.6866608424 $\mathrm{H}, 0,1.3290572112,17.0878305202,13.7638228838$ $\mathrm{H}, 0,2.0632813872,16.3169490101,15.1679489605$ C,0,1.0730217685,18.1803002661,15.6383338005 $\mathrm{H}, 0,0.0640535419,17.7541996344,15.7082903962$ H,0,0.9506452519,19.1870319538,15.219322294 C,0,1.6460733193,18.2717063809,17.0637155244 H,0,1.9030903461,17.269979433,17.4222735844 $\mathrm{H}, 0,0.9048924566,18.704580208,17.7438387477$ $\mathrm{N}, 0,6.606591657,17.0418508089,15.1839068815$ $\mathrm{H}, 0,6.9852460996,16.461826439,15.9346683228$ H, $0,6.2296456356,16.3886088813,14.4970780077$ $\mathrm{N}, 0,6.1699814832,18.1921333405,17.8981540478$ H,0,7.1582317144,18.3907454192,17.7132392077 H,0,5.8511650169,18.9189290076,18.5354349517 O,0,7.6562907311,19.9106574653,15.7656672339 $\mathrm{O}, 0,8.9314397135,18.2430467889,16.5971079374$ O,0,4.2913401422,16.4008238629,16.9062754137 O,0,4.3673668331,15.1538818457,18.7699921546 P,0,3.2104235129,19.2723304627,17.1536247402 $\mathrm{P}, 0,3.5903932331,17.8870707081,14.2392842284$ Ru,0,4.9392789248,18.2292235737,16.064553773 H,0,6.2770701817,19.6697789188,15.589701709 H,0,5.3601934982,19.7372067063,15.3944918325

\section{$\mathbf{T S}_{4-6}$}

C,0,7.5641867405,18.147699577,14.7007516976 C, $0,8.6513454073,17.4663656061,13.8792298339$ H,0,9.3580017099,18.2194230539,13.5218901961 H,0,9.1866650413,16.761174165,14.5391741284 C,0,4.9729557824,16.0943794509,18.13955769 C,0,6.2810583396,16.861431316,18.4386404678 H,0,6.5618891399, 16.69582364,19.4825447949 H,0,7.0516022165,16.4377102663,17.7878677815 C,0,3.3080793909,19.4569739657,19.0626112445 C,0,3.2360286194,18.2944893879,19.8506927887 $\mathrm{H}, 0,2.9584060455,17.3436670618,19.4083967687$ C,0,3.5613567688,18.3389004274,21.2072602559 $\mathrm{H}, 0,3.5060193768,17.4286129961,21.7975709804$ C,0,3.967670646,19.5390615469,21.7951665933 $\mathrm{H}, 0,4.2238978039,19.5707965551,22.8506603631$ C,0,4.0495005185,20.6971137072,21.0185022652 $\mathrm{H}, 0,4.3666567789,21.6345076878,21.4679767589$ C,0,3.7260946257,20.6569605218,19.660473052
H,0,3.7937537911,21.5628423809,19.0646492748 C,0,2.4577855668,20.9823881845,16.7409099206 C,0,2.9834158061,21.6443556775,15.6247901674 $\mathrm{H}, 0,3.8143436565,21.208949072,15.0845148084$ C, $0,2.4350091333,22.8553439202,15.1952847603$ H,0,2.8468763762,23.3442841837,14.3170281645 C, $0,1.3628767307,23.4214111903,15.8837754971$ H,0,0.9359386808,24.3639122584,15.5510151329 C, $0,0.836690065,22.7737686862,17.0062991387$ $\mathrm{H}, 0,0.0046627831,23.2134274873,17.55007343$ C,0,1.3772897242,21.561192125,17.4296469483 H,0,0.9662465297,21.0683558501,18.3070880495 C,0,4.3878527336,16.5679575434,13.3578001116 C, $0,4.785960849,15.413708465,14.0551363988$ $\mathrm{H}, 0,4.7118554828,15.3861531728,15.138685937$ C,0,5.290042497,14.3159294005,13.3581134922 $\mathrm{H}, 0,5.5942187495,13.4277145412,13.9056913203$ C,0,5.4151706489,14.3618295841,11.9656091226 H,0,5.8155862376,13.5074697536,11.4257039115 C,0,5.028423174,15.5090434655,11.2722316522 H,0,5.1309063334,15.5551066908,10.1913414783 C,0,4.5139449689,16.6077050507,11.9640569072 $\mathrm{H}, 0,4.2257563125,17.4979933345,11.4145035078$ C,0,4.6328924877,20.0097327989,12.7025852225 H,0,5.6103967126,19.7015117953,13.0613640624 C,0,4.5307106963,21.0961114334,11.8357997417 H,0,5.4312020699,21.6169993425,11.5218921576 C,0,3.2778993618,21.5165910855,11.3784769685 H,0,3.1986762302,22.3708034057,10.7112099469 C,0,2.1308656275,20.8308362928,11.7774448706 $\mathrm{H}, 0,1.1535125667,21.147527957,11.4230864357$ C,0,2.2319857843,19.7339419984,12.636884346 $\mathrm{H}, 0,1.3267367376,19.2140695048,12.930855226$ C,0,3.4802750109,19.3202770568,13.1193888836 C,0,1.991782735,17.3068125701,14.6917286021 $\mathrm{H}, 0,1.4959361675,17.102990453,13.7354863569$ $\mathrm{H}, 0,2.1818547377,16.3378764131,15.1679261422$ C,0,1.0889856194,18.1558619339,15.6072494261 H,0,0.0995053684,17.6809874162,15.6239245928 H,0,0.9372762859,19.1584865402,15.1880841587 C,0,1.5785784061,18.2694868532,17.0623457262 H,0,1.8522307488,17.2795696129,17.4402150991 $\mathrm{H}, 0,0.7870086804,18.681752441,17.6976707464$ $\mathrm{N}, 0,7.9824785508,16.8477272069,12.728113146$ 
H,0,7.3298964125,16.1393536183,13.060533521 $\mathrm{H}, 0,8.6681469104,16.3679304093,12.1483203795$ N,0,6.173079285,18.308602993,18.1074205776 $\mathrm{H}, 0,7.0935643797,18.7107193316,17.9393377527$ H,0,5.7546326953,18.8194955527,18.8836397438 O,0,6.7884728169,17.3872068355,15.3486479063 $\mathrm{O}, 0,7.4625714898,19.4172888008,14.6378644428$ O,0,4.2776426298,16.526085629,17.1207691794 O,0,4.6772707115,15.1263807621,18.8287949325 $\mathrm{P}, 0,3.0992179734,19.3260916654,17.2346534732$ P,0,3.6986565881,17.965822672,14.346520442 $\mathrm{Ru}, 0,4.8979868533,18.4007728051,16.243591342$ $\mathrm{H}, 0,6.3029674896,19.7250374106,15.2854109352$ H,0,5.4792033965,20.037426452,15.7023677212

\section{Int 6}

C,0,7.556364436,17.8974850211,14.724381906 C,0,8.6071200061,17.1661179375,13.9169446596 $\mathrm{H}, 0,9.4664104066,17.8252820943,13.7667433105$ H,0,8.9261988391,16.2856463582,14.4972944677 C,0,5.0221006487,16.0479857759,18.1397510228 C,0,6.3011639893,16.8549927035,18.4749618195 $\mathrm{H}, 0,6.5270656886,16.7462105844,19.5395038287$ $\mathrm{H}, 0,7.1166723248,16.4122288891,17.8951985053$ C,0,3.3827583405,19.4857803439,19.0488074689 C,0,3.3361040647,18.340229508,19.8641489424 $\mathrm{H}, 0,3.0991301506,17.3700893214,19.4395811257$ C,0,3.6294713604,18.4281386836,21.2259074674 H,0,3.591979595,17.5309319901,21.8374850368 C,0,3.9794166471,19.6559072077,21.7929747645 H,0,4.2101966656,19.7219521339,22.8528294739 C,0,4.0370042534,20.7970841566,20.9900564205 $\mathrm{H}, 0,4.3101701641,21.7559165333,21.4231525617$ C,0,3.7445581522,20.713098628,19.6268279026 $\mathrm{H}, 0,3.7911835366,21.6065963708,19.0106763818$ C,0,2.5508036534,20.9482580178,16.6876859786 C,0,3.1605917085,21.6352295383,15.631334975 $\mathrm{H}, 0,4.0477302128,21.2174108174,15.1715048021$ C,0,2.6261288926,22.8390369303,15.1657980368 $\mathrm{H}, 0,3.1021643144,23.3463538198,14.3313821378$ C,0,1.4834147426,23.3737292168,15.7596001991 H,0,1.0652205752,24.3092193601,15.3968874276 C, $0,0.8756968168,22.7041846262,16.8267153599$ H,0,-0.0103015031,23.1207407404,17.2989400144
C,0,1.4040339692,21.4989762033,17.2856113392 $\mathrm{H}, 0,0.9288518793,20.9897978168,18.1202236275$ C,0,4.410181627,16.5996493674,13.305823611 C,0,4.8121578107,15.4235898024,13.9647339838 $\mathrm{H}, 0,4.7559301047,15.3691596724,15.0489745837$ C,0,5.2955990541,14.3397271866,13.2311336317 H,0,5.6012286141,13.4350813465,13.7504386048 C, $0,5.3938193138,14.4184345848,11.8374592508$ H,0,5.7752309272,13.5741815221,11.2685657427 C,0,5.0013318474,15.5860325971,11.1806819671 H,0,5.0794984362,15.6570389422,10.0989367481 C,0,4.5092007082,16.6711220899,11.9106399544 $\mathrm{H}, 0,4.2129048263,17.5753891043,11.3887325364$ C,0,4.598010927,20.060955701,12.7106432386 $\mathrm{H}, 0,5.5887125436,19.7698891413,13.0461577542$ C, $0,4.4557386143,21.1500027171,11.8532264735$ H,0,5.3374828107,21.6928883573,11.5230934749 C,0,3.1846028158,21.5464967752,11.4262840304 $\mathrm{H}, 0,3.0719414553,22.4041335998,10.768279204$ C,0,2.0630665916,20.8314621596,11.8443893811 $\mathrm{H}, 0,1.0711615092,21.1287596019,11.5143970994$ C,0,2.2075019637,19.7296586006,12.6914772213 H,0,1.3201728305,19.1868023005,12.9975061899 C,0,3.4731556258,19.3401306684,13.1480228292 C,0,2.0536143235,17.2812444642,14.7110345241 $\mathrm{H}, 0,1.546203145,17.0791579822,13.7603418515$ $\mathrm{H}, 0,2.2658532555,16.31122818,15.1757423747$ C,0,1.1497585681,18.1043750862,15.648193506 $\mathrm{H}, 0,0.1750612389,17.6006227154,15.6895842858$ $\mathrm{H}, 0,0.9583082565,19.1014543356,15.2325505908$ C,0,1.6722254933,18.2364221067,17.0892059122 H,0,1.9680195999, 17.252360891,17.4668121874 $\mathrm{H}, 0,0.8884641589,18.6389922357,17.7402100265$ $\mathrm{N}, 0,7.9998436426,16.873133926,12.6140756507$ $\mathrm{H}, 0,7.2814066581,16.1589823754,12.7207446188$ $\mathrm{H}, 0,8.7040803234,16.4951044857,11.9846267765$ N,0,6.1898165036,18.2844329635,18.071025601 H,0,7.1129467656,18.6908628395,17.9279729551 H,0,5.7499776074,18.8216414595,18.8171645021 O,0,6.7334329689,17.2659109515,15.40595376 O,0,7.5255069888,19.2010503362,14.5947233156 O,0,4.3697540573,16.4414607961,17.0890735046 O,0,4.7336564823,15.0911585755,18.8522728528 $\mathrm{P}, 0,3.1929598222,19.3024770346,17.220174111$ 
$\mathrm{P}, 0,3.7487655417,17.9730830766,14.3563778528$

$\mathrm{Ru}, 0,4.9740543962,18.3445966586,16.2439891519$ H,0,6.6688269086,19.5078087803,15.1098497199 H,0,5.5139634356,19.8399527974,15.7669573227

\section{TS5-7}

C,0,4.402092898,0.0549880901,-0.1742913893

C,0,3.8457282866,-1.230738756,-0.8046139425 H,0,4.1328300744,-1.1743718407,-1.8612572994 $\mathrm{H}, 0,4.4303900076,-2.0442873543,-0.3686302429$ C,0,1.1802516484,-2.7530054989,1.7141898187 C,0,1.6430280686,-1.7598866469,2.8077523751 $\mathrm{H}, 0,0.8353369566,-1.6066434771,3.5258638883$ $\mathrm{H}, 0,2.5290502416,-2.136925444,3.325471585$ C,0,-0.7727772141, $0.9112618878,3.1630055028$ C,0,-1.205147467,-0.0005763565,4.1372239839 $\mathrm{H}, 0,-1.7913118689,-0.8684680989,3.8516851399$ C,0,-0.8793503984,0.1901013021,5.4825846857 $\mathrm{H}, 0,-1.226870668,-0.5227243551,6.2257173338$ C,0,-0.1078729798,1.286257652,5.8708520648 $\mathrm{H}, 0,0.1481305095,1.4305832474,6.9168159337$ C,0,0.3332692794,2.1982593026,4.9079865809 $\mathrm{H}, 0,0.9319581758,3.0561256702,5.2022373609$ C, $0,0.002108497,2.013454493,3.56525297$ $\mathrm{H}, 0,0.3391727822,2.7323595155,2.8221366075$ C,0,-1.6823135275,2.2908892363,0.8370307025 C,0,-1.0548554826,3.0037483636,-0.1902421614 $\mathrm{H}, 0,-0.1689482708,2.5892879784,-0.6537378269$ C,0,-1.5679140427,4.2297026502,-0.6205844433 $\mathrm{H}, 0,-1.0763307604,4.7587566527,-1.4321741183$ C,0,-2.708841494,4.7600332118,-0.0199924702 $\mathrm{H}, 0,-3.1113143399,5.7123332621,-0.355444334$ C,0,-3.3328866565,4.065371351,1.0213185453 $\mathrm{H}, 0,-4.2159623487,4.4793906029,1.5011192174$ C,0,-2.8229008947,2.840561421,1.4470469355 H,0,-3.3095513837,2.3160047335,2.2653703283 C,0,-0.1313131804,-2.041952223,-2.6571418748 C,0,0.151678914,-3.2883299797,-2.0642856221 H,0,0.062399568,-3.4022724669,-0.9847047298 C, $0,0.5864483796,-4.3563671512,-2.8488132876$ $\mathrm{H}, 0,0.8071913269,-5.3101166443,-2.3771840282$ C,0,0.7497145622,-4.1961782119,-4.2277616365 H,0,1.0940669261,-5.0280116653,-4.8365428744 C, $0,0.4757335708,-2.9624227266,-4.819990262$
H,0,0.6026087994,-2.8315735326,-5.891584448 C,0,0.0361833627,-1.8888353293,-4.0407004539 H,0,-0.1781704179,-0.93521134,-4.51329978 C,0,0.0970352299,1.5095367746,-3.064956296 $\mathrm{H}, 0,1.0813813752,1.2763723425,-2.66868923$ C,0,-0.0709693704,2.6041818736,-3.9105610015 $\mathrm{H}, 0,0.7903773329,3.2016334262,-4.1974300113$ C,0,-1.3464394612,2.9418616037,-4.3723991353 $\mathrm{H}, 0,-1.4821784527,3.8044381399,-5.0192711874$ C,0,-2.4440689897,2.1672297341,-3.9980328578 H,0,-3.4390241295,2.4238240846,-4.3518103778 C,0,-2.271330381,1.058356097,-3.1660106641 $\mathrm{H}, 0,-3.1398611007,0.4678452838,-2.8945396452$ C,0,-1.0011124828,0.7225645882,-2.6798062589 C,0,-2.3662640164,-1.3449439192,-1.0827789854 $\mathrm{H}, 0,-2.9291114675,-1.528730152,-2.0051961179$ $\mathrm{H}, 0,-2.1527872629,-2.3243120627,-0.6394444082$ C,0,-3.1966200655,-0.5061727024,-0.0962341785 $\mathrm{H}, 0,-4.1830918718,-0.9795486723,-0.0076053823$ H,0,-3.3750542066,0.4992359898,-0.4972133267 C,0,-2.5932394544,-0.4010510059,1.3164399214 $\mathrm{H}, 0,-2.2941464957,-1.3957425016,1.6651858053$ H,0,-3.335507739,0.0040963988,2.0128991649 $\mathrm{N}, 0,2.3918065953,-1.5370810075,-0.7011779575$ $\mathrm{H}, 0,2.3011148146,-2.4480347842,-0.2400105666$ H,0,2.0355934981,-1.691696757,-1.6400474828 $\mathrm{N}, 0,1.9140912857,-0.4540556128,2.1356965418$ H, $0,2.9020948702,-0.3784802946,1.903146403$ $\mathrm{H}, 0,1.7226472481,0.3217304625,2.7685430518$ $\mathrm{O}, 0,5.5913365527,0.126953383,0.044932315$ O,0,3.5800055879,1.0790813584,0.0674087791 $\mathrm{O}, 0,0.0936025067,-2.39819965,1.110534113$ O,0,1.8986839704,-3.7156461346,1.420403902 $\mathrm{P}, 0,-1.046980318,0.6341489614,1.3571201807$ $\mathrm{P}, 0,-0.6816294746,-0.6800344999,-1.5258625839$ $\mathrm{Ru}, 0,0.6638024126,-0.373082768,0.286926203$ H,0,2.6062527807,0.8662367491,-0.0984693455 H, $0,1.1436582398,1.0639827489,-0.282146715$

\section{Int 7}

C,0,7.9676018757,18.6823967477,15.0489348281 C,0,7.2712402052,17.5998165279,14.2279256417 $\mathrm{H}, 0,6.6298806505,18.0110580439,13.4462521741$ H,0,8.0588959054,16.996479254,13.7488718895 
C, $0,4.777766502,15.7817191624,18.0629088952$ C,0,6.0128398081,16.641898674,18.4516379819 H,0,6.0607490671,16.697416592,19.5420359232 H,0,6.9054168959, 16.0973779354,18.1211273082 C,0,3.2961376752,19.2520773203,18.903832232 C,0,3.1809033343,18.0771705036,19.6703820263 H,0,2.9012593023,17.1375600691,19.2059333587 C,0,3.4577593534,18.0890042227,21.0374278185 $\mathrm{H}, 0,3.3705341093,17.1663204436,21.6043690803$ C,0,3.8589373591,19.2710441241,21.6645898835 $\mathrm{H}, 0,4.0801585793,19.2780077499,22.7285240722$ C,0,3.9781492061,20.4419165192,20.914783525 $\mathrm{H}, 0,4.2877363303,21.3676178167,21.393215907$ C,0,3.7001003062,20.4337224338,19.5451601342 H,0,3.7960636474,21.3541424467, 18.9775701185 C,0,2.7563076322,20.8946923963,16.585685022 C,0,3.8517366171,21.7241229131,16.293516386 $\mathrm{H}, 0,4.8527256227,21.3033311938,16.3296748266$ C,0,3.662653058,23.05628778,15.9272725589 $\mathrm{H}, 0,4.5239449893,23.6830614309,15.7098154326$ C,0,2.3707442637,23.5783349424,15.8267288417 $\mathrm{H}, 0,2.2198868579,24.6121565625,15.5272365345$ C, $0,1.2744126047,22.7650550537,16.1145309908$ $\mathrm{H}, 0,0.2657723065,23.1631522846,16.0402609597$ C,0,1.4660699016,21.4368737239,16.5020543382 H,0,0.5970979997,20.8294724952,16.7323930071 C,0,4.0106402624,16.2493338819,13.1928386188 C,0,4.0898531833,14.9841297904,13.8089464238 $\mathrm{H}, 0,3.7674592517,14.8659767324,14.8416223742$ C,0,4.6303106975,13.8958937222,13.1220904944 H,0,4.6815762473,12.926367631,13.6106770026 C,0,5.1113067589,14.0527689766,11.8184925405 $\mathrm{H}, 0,5.5337602555,13.2046635962,11.2864028873$ C,0,5.0502231337,15.3054866678,11.2069333393 H,0,5.4252131862,15.4369104573,10.1949936569 C,0,4.5054117225,16.3974300521,11.8893994861 $\mathrm{H}, 0,4.4584886154,17.3650346363,11.3981542603$ C,0,3.5726856695,20.2691435997,13.1924591855 H,0,4.2425295694,20.4329412228,14.0288535254 C,0,3.2320943147,21.3169265767,12.3339067244 $\mathrm{H}, 0,3.6386817325,22.3091905062,12.5108802541$ C,0,2.3600123593,21.0917280048,11.2688976626 H,0,2.0916599772,21.9052445102,10.599810903 C,0,1.8275981431,19.8147790292,11.0651637067
H,0,1.1508530312,19.6328982854,10.2341880977 C,0,2.1583689778,18.773080683,11.9309394175 H,0,1.7407304349,17.7844050799, 11.7590392583 C,0,3.0355325763,18.9892221701,13.0076659588 C, $0,1.7165680075,17.0757815909,14.688260501$ H,0,1.1704467147,16.8169286476,13.7744377773 $\mathrm{H}, 0,1.8530694815,16.1562345235,15.2684130027$ C,0,0.9154674254,18.0973106392,15.5138961372 H,0,-0.1192821064,17.7374374411, 15.584778131 H,0,0.8680146804,19.0557980104,14.9806255768 C,0,1.4417959954,18.2988125322,16.9469424243 $\mathrm{H}, 0,1.5994772697,17.3184951851,17.408964265$ $\mathrm{H}, 0,0.7086699132,18.8328322667,17.5616520446$ $\mathrm{N}, 0,6.4314262871,16.8157042989,15.1445511363$ H,0,7.0254037101,16.443404822,15.8861037059 $\mathrm{H}, 0,6.0337952228,16.0111566106,14.6587666271$ $\mathrm{N}, 0,6.0285142648,17.9976930469,17.8350684795$ H,0,6.9792110633,18.2833096391,17.5976506214 $\mathrm{H}, 0,5.6770360537,18.6780560757,18.5057025602$ O,0,8.5153149121,18.4242345577,16.0987679498 O,0,8.0337461591,19.9185034012,14.5197646293 O,0,4.1610031359,16.1144906662,16.9738469951 O,0,4.4990860114,14.8372501568,18.7976441729 $\mathrm{P}, 0,3.098454271,19.1397909752,17.0614996271$ $\mathrm{P}, 0,3.4308850205,17.6647440303,14.2379284445$ $\mathrm{Ru}, 0,4.7630969545,18.0037583249,16.0336259625$ H,0,7.358115591,20.0084620867,13.8265724814 H,0,5.4085249845,19.3675443353,15.4209947724

TS8-10

C,0,8.4040971972,17.5828996618,15.8919007362 C,0,7.5664134722,17.1835980244,14.6756536398 H,0,7.3036679543,18.0483622634,14.0673640454 $\mathrm{H}, 0,8.1860380838,16.5032486815,14.0721082479$ C,0,4.2897574742,15.5469589509,17.9747651456 C,0,5.610355728,16.1680838394,18.490528662 H,0,5.6014140976,16.1272943577,19.5824448632 H,0,6.4315149956,15.5288608065,18.1488109765 C,0,3.2867613724,18.9842905364,19.002219171 C,0,2.9360733568,17.8349945194,19.7293405312 H,0,2.4297659754,17.0071444708,19.2459101262 C,0,3.2800766263,17.7148820938,21.0771825889 $\mathrm{H}, 0,3.0110736707,16.8102271685,21.6148199672$ C,0,3.9810908912,18.7369437911,21.7192741522 
$\mathrm{H}, 0,4.2544688565,18.6385470443,22.7661895429$ C,0,4.3360903524,19.8850943577,21.0063866813 $\mathrm{H}, 0,4.8815218726,20.6866071333,21.4975642654$ C,0,3.994062568,20.0070225772,19.6585506608 H,0,4.2742264703,20.9050451977,19.115257096 C,0,2.6365282637,20.7938196851,16.8454478 C,0,3.0307499529,21.3859624799,15.6403483808 H,0,3.6343025826,20.8193160741,14.9459655609 C,0,2.6394550563,22.6875172685,15.3189210244 $\mathrm{H}, 0,2.9502881405,23.119320051,14.3714970003$ C,0,1.851451011,23.4170807685,16.2086180583 $\mathrm{H}, 0,1.5479462754,24.4319763642,15.9657061222$ C,0,1.4481796155,22.8360469336,17.4150550389 $\mathrm{H}, 0,0.8314522253,23.3986199141,18.111130533$ C,0,1.8311545553,21.5326715939,17.7289525214 $\mathrm{H}, 0,1.510632488,21.0920846042,18.669111559$ C,0,3.8523962722,16.3005167901,13.1236166738 C,0,3.8472091608,15.0299609981,13.7338116054 H,0,3.5998798917,14.9408746122,14.790500011 C,0,4.1955147,13.8959851073,12.9992156486 $\mathrm{H}, 0,4.1868548213,12.922162523,13.4816592588$ C,0,4.5603011629,14.01186149,11.6546868846 $\mathrm{H}, 0,4.8355841723,13.1278603145,11.0857852507$ C,0,4.5733673635,15.2679188735,11.0473655947 $\mathrm{H}, 0,4.8572675964,15.3656748281,10.0027282581$ C,0,4.22062229,16.4075510048,11.7757599086 $\mathrm{H}, 0,4.2324456415,17.3780703533,11.2896256051$ C,0,4.5920532043,19.7688062904,12.6619047183 H,0,5.5197846695,19.3971043807,13.0847796245 C,0,4.6260168697,20.8811544416,11.8221003634 H,0,5.5813389371,21.3419123955,11.5862237485 C,0,3.4373173101,21.4066865018,11.3076576937 $\mathrm{H}, 0,3.4599368181,22.2817373513,10.6636068416$ C,0,2.2202038479,20.8025030189,11.6259200059 $\mathrm{H}, 0,1.2915320862,21.2046258955,11.2296558788$ C,0,2.1870623034,19.6803965323,12.4575110476 $\mathrm{H}, 0,1.2282626693,19.2303712614,12.6921970238$ C,0,3.371399202,19.1563645484,12.9937984193 C,0,1.6421117051,17.3388532694,14.5428363899 $\mathrm{H}, 0,1.1078560575,17.2269107559,13.592587321$ $\mathrm{H}, 0,1.6909045843,16.3454670066,15.0016145095$ C,0,0.8934984955,18.2935857644,15.4889562859 H,0,-0.1655377408, 18.005569405,15.481902172 H,0,0.9305858891,19.324513734,15.1140140084
C,0,1.3863476924,18.2423508087,16.9456051465 H,0,1.4805288992,17.199288977, 17.2646468167 H,0,0.6766923387,18.7513797426,17.6074739319 $\mathrm{N}, 0,6.3373578052,16.5284212843,15.1553314389$ $\mathrm{H}, 0,6.597839198,15.849658246,15.8722741695$ H,0,5.9047712371,15.9974684312,14.4014406019 $\mathrm{N}, 0,5.87819046,17.5495803275,18.0015564939$ H,0,6.8832535866,17.6624883163,17.8744145338 H,0,5.5858303173,18.2219794074,18.7068593372 O,0,8.9977664752,18.7624214922,15.8686643988 $\mathrm{O}, 0,8.5177351328,16.8097127815,16.8322405398$ O,0,3.8173955894,16.0135450744,16.8549039471 O,0,3.7908665452,14.6345890919,18.625969727 $\mathrm{P}, 0,3.0626121731,19.0248950056,17.1682033886$ $\mathrm{P}, 0,3.4235274772,17.7552410114,14.1915634446$ $\mathrm{Ru}, 0,4.7051237416,17.8952513707,16.0686835706$ H,0,8.5177450302,19.3911144097,15.2206230318 H,0,5.5317923186,19.3198790174,15.5401415892 C,0,6.4930958656,20.596865994,15.2612952695 H,0,5.6291100425,21.1088414493,14.8156809432 O,0,7.4193020496,20.2474158232,14.4786781212 C,0,6.7571150097,20.9512425046,16.7122141443 H,0,7.2811675803,21.9170256972,16.7329051105 H,0,5.8172402987,21.0445239507,17.2601781824 H,0,7.397773973,20.2083301239,17.1919166704

\section{TS9-11}

C,0,7.8087148499,17.9667792908,14.4525303639 C, $0,8.340675633,16.9018391892,13.5008692441$ H,0,9.3885391876,17.1183198993,13.2800305091 H,0,8.27059233,15.9208364918,13.9983496067 C,0,5.4155167718,16.6742094523,17.9924927493 C,0,6.5964920537,17.6521938484,18.1658700138 H,0,6.9413018435,17.6145792356,19.2029217331 $\mathrm{H}, 0,7.4016516498,17.3033350065,17.5138421282$ C,0,3.3871424927,19.8135847993,18.8921116672 C,0,3.4347423836,18.6655243103,19.7022899092 H,0,3.2034520578,17.6902636183,19.2873707607 C,0,3.8277793753,18.7585106116,21.0378961969 H,0,3.8669438236,17.8588031205,21.6452435687 C,0,4.1858720578,19.9941157642,21.582270092 H,0,4.4970469058,20.063494905,22.6209430042 C,0,4.1517046359,21.1387534057,20.7830534753 H,0,4.4334073198,22.1031332964,21.1973933975 
C,0,3.7596366978,21.0498689056,19.4455005968 H,0,3.7381887731,21.946317126,18.8326613049 C,0,2.3164401139,21.1955503083,16.5574525989 C,0,2.5750707939,21.7081034397,15.280098416 H,0,3.3126829136,21.2282548925,14.6493166289 C,0,1.8893226112,22.8297869027,14.8092720511 H,0,2.0986218282,23.2012946046,13.8100509511 C,0,0.9425413681,23.4591224057,15.617518834 $\mathrm{H}, 0,0.4113908021,24.3356861562,15.2561756094$ C,0,0.6749340863,22.9560707203,16.8942092768 $\mathrm{H}, 0,-0.0633538208,23.440773174,17.5275270973$ C,0,1.3498647308,21.827325837,17.3577290631 $\mathrm{H}, 0,1.1299506048,21.4405480163,18.3491108125$ C,0,4.2532696943,16.5966471002,13.3672715664 C,0,4.8090283057,15.5606548832,14.1370890988 H,0,4.9316129588,15.6931777331,15.2071190343 C,0,5.2140907171,14.3746944839,13.523070581 H,0,5.6492734422,13.58276211,14.1266849222 C,0,5.0714981081,14.2086223167,12.1426516708 H,0,5.3959005682,13.2864486429,11.6674560303 C,0,4.5093290114,15.2308000457,11.3751585738 $\mathrm{H}, 0,4.3937145334,15.1087400624,10.3013509567$ C,0,4.0992959261,16.4194144902,11.9843809249 $\mathrm{H}, 0,3.6756344473,17.2128663739,11.3762626075$ C,0,4.6736203254,19.8407693481,12.3186706342 H,0,5.6327817267,19.4450565208,12.6441360346 C,0,4.6324494132,20.8388991602,11.3475538038 H,0,5.5600924474,21.218306512,10.9277395374 C,0,3.4033062064,21.3501834554,10.9172010805 H,0,3.3725588157,22.1354468046,10.1666184526 C,0,2.2175868725,20.8433652802,11.4491520249 $\mathrm{H}, 0,1.258936538,21.2315241616,11.1150717654$ C,0,2.2560445725,19.8332099489,12.4149581629 $\mathrm{H}, 0,1.3226484365,19.4561271464,12.821103425$ C,0,3.4822381816,19.3300587198,12.8664345908 C,0,1.9399495249,17.5037914997,14.6291050012 H,0,1.417748249,17.3091993512,13.6846776879 H,0,2.1239619371,16.5270405199,15.0922499899 C,0,1.0692309623,18.3474904134,15.5738939074 $\mathrm{H}, 0,0.0891731476,17.8582081834,15.6443449352$ $\mathrm{H}, 0,0.8810813219,19.3422601085,15.1515170955$ C,0,1.6342349293,18.4816229933,16.9997761513 $\mathrm{H}, 0,1.9627891833,17.5041285173,17.3674346254$ $\mathrm{H}, 0,0.8661138281,18.8708125668,17.6776365355$
$\mathrm{N}, 0,7.558305019,17.0085573038,12.2602458697$ H,0,6.6366888199,16.6115362373,12.4203465397 $\mathrm{H}, 0,7.9912752845,16.4378356842,11.5369813478$ $\mathrm{N}, 0,6.2328067079,19.0260679193,17.7423844694$ $\mathrm{H}, 0,7.0241400137,19.5837755352,17.3958223265$ $\mathrm{H}, 0,5.8192783417,19.5333705715,18.5229884611$ O,0,6.7512452939,17.7157902099,15.0750307843 O,0,8.4855417908,19.0568481093,14.4950821554 O,0,4.5645918782,16.9697531991,17.0386684729 O,0,5.3345941205,15.682936704,18.7040156219 P,0,3.1082401894,19.6137175431,17.0822991233 $\mathrm{P}, 0,3.6583061256,18.1157656832,14.2364357022$ $\mathrm{Ru}, 0,4.9189157778,18.7910506722,16.0451304156$ $\mathrm{H}, 0,8.0224916995,19.8883513993,15.209425834$ H,0,5.3960419501,20.3408151196,15.4141147445 C,0,6.4039680702,21.2459990543,15.4345334578 $\mathrm{H}, 0,6.4320537327,21.3625862885,14.3375306841$ O,0,7.5148355008,20.8000843364,15.9926455938 C,0,5.7841744352,22.428985908,16.1562962589 H,0,4.8619094582,22.7721713784,15.6845438308 $\mathrm{H}, 0,5.575987929,22.1632216321,17.1972495278$ H,0,6.516575845,23.2446189539,16.1520334994

\section{Int 10}

C, $0,8.2887009643,17.7673271471,15.7414011565$ C,0,7.3794710596,17.2162321191,14.6101482806 H,0,7.0486517925,18.0211010502,13.9520881895 H,0,7.9614937732,16.502250418,14.0113887336 C, $0,4.2114307153,15.6128419613,17.9263094724$ C, $0,5.4826121581,16.2499556174,18.5140184341$ H,0,5.3736412364,16.2796708609,19.5999390062 H,0,6.3210808494,15.5813966232,18.2903684554 C,0,3.1832479654,18.9352339898,19.0574885718 C, $0,2.7900057858,17.8104025481,19.7990708002$ H,0,2.2448594379, 16.9987815608,19.3300847919 C,0,3.1338390015,17.6951685052,21.1479257783 $\mathrm{H}, 0,2.8267965143,16.8124633802,21.701544671$ C,0,3.8800757441,18.6947681463,21.7733685328 H,0,4.1534898778,18.5986407811,22.8203288759 C, $0,4.2781887281,19.8188101317,21.0446517453$ H,0,4.8584918459,20.6032916117,21.5226688858 C,0,3.9331456207,19.9380712143,19.6981055677 H,0,4.2407145876,20.8201468966,19.1433360284 C,0,2.4972691371,20.7505617078,16.9153861349 
C,0,2.8239220958,21.3424392467,15.6887065342 H,0,3.3935589736,20.7879106362,14.956354858 C,0,2.4088438263,22.6414781892,15.3872330004 $\mathrm{H}, 0,2.6717919828,23.0734800154,14.4258600116$ C,0,1.6629759362,23.3676765857,16.3147880636 H,0,1.3427024293,24.3807124754,16.0867542089 C,0,1.3248201843,22.7858380578,17.5403666267 $\mathrm{H}, 0,0.7403136314,23.3450157943,18.2661361216$ C,0,1.7327908101,21.4862904408,17.8373256873 $\mathrm{H}, 0,1.4628855046,21.0467412601,18.7932721871$ C,0,3.793631491,16.2657192186,13.1263273936 C, $0,3.777776739,14.9800680365,13.7021429283$ $\mathrm{H}, 0,3.4947366477,14.8567324079,14.7453449364$ C,0,4.1572422349,13.868339661,12.9496069502 $\mathrm{H}, 0,4.1408605097,12.8820341637,13.4054447403$ C,0,4.5636906282,14.0229032796,11.6212738642 $\mathrm{H}, 0,4.863648324,13.1563079818,11.0385543637$ C,0,4.5874686269,15.2947528421,11.0483277963 $\mathrm{H}, 0,4.9048764083,15.4224683761,10.0168729144$ C,0,4.2047860682,16.4121162151,11.7950040766 $\mathrm{H}, 0,4.227712282,17.3959530258,11.3368007541$ C,0,4.4680939056,19.8061138139,12.7880946114 $\mathrm{H}, 0,5.4037257008,19.4655011989,13.2181822847$ C,0,4.4768682769,20.9605747825,12.0063701018 $\mathrm{H}, 0,5.4174540572,21.4759624077,11.8353688242$ C,0,3.2824596201,21.4533877072,11.4745335249 $\mathrm{H}, 0,3.2841513203,22.3593600943,10.8744129933$ C, $0,2.0849273453,20.7775581143,11.7176378532$ $\mathrm{H}, 0,1.1526600112,21.1544601916,11.3055950956$ C,0,2.075806994,19.6185622775,12.4965976861 $\mathrm{H}, 0,1.1316343146,19.1160755727,12.6788025997$ C,0,3.266580289,19.1260150762,13.0500278988 C, $0,1.5700217518,17.2612540318,14.5769001444$ H,0,1.031460789,17.1448192156,13.6298642742 $\mathrm{H}, 0,1.6268254505,16.2681252874,15.0340251266$ C,0,0.8177590092,18.2137608534,15.5226458772 H,0,-0.2399809476,17.9220417305,15.51230313 $\mathrm{H}, 0,0.8517665077,19.2439681643,15.1460587361$ C,0,1.3020693856,18.1648363096,16.9828466377 H,0,1.4168416921,17.1221591683,17.2953757888 $\mathrm{H}, 0,0.5762852563,18.6530211023,17.6427686874$ $\mathrm{N}, 0,6.2076733481,16.5532177123,15.2291260895$ $\mathrm{H}, 0,6.5999949358,16.0034807811,15.9983002551$ H,0,5.7744903042,15.8977194041,14.5799787064
$\mathrm{N}, 0,5.7993244992,17.5938617705,17.9606283025$ $\mathrm{H}, 0,6.803995116,17.6185076447,17.7175738$ H,0,5.6187060898,18.3088877551,18.6614501399 O,0,8.9356801272,18.8139793088,15.4891378982 $\mathrm{O}, 0,8.2726307013,17.1040886155,16.8141831061$ $\mathrm{O}, 0,3.7549477038,16.1248612773,16.8018518821$ O,0,3.6911909602,14.6610821363,18.4876791531 $\mathrm{P}, 0,2.9519875423,18.9898863788,17.2286177681$ $\mathrm{P}, 0,3.3398272563,17.6969140864,14.20483655$ $\mathrm{Ru}, 0,4.6372605777,17.8668515167,16.1099009354$ H,0,7.9440824681,19.9133565432,14.7436996983 H,0,5.7255419467,19.4673444516,15.6042993906 C,0,6.2631100995,20.4880488082,15.5296246072 $\mathrm{H}, 0,5.4598477055,21.185679877,15.2861412459$ O,0,7.153675352,20.4729684503,14.4717350246 C,0,6.8647285085,20.7911798619,16.8963993751 H,0,7.3009791577,21.7958606021,16.8838891975 H,0,6.0873646219,20.7533260501,17.6674830599 H,0,7.6564495096,20.0781442276,17.1386899384

\section{Int 11}

C,0,7.2655637406,17.8845228372,14.0773889522 C,0,8.0994451133,16.7461508836,13.473167958 H,0,7.602049469, 16.4758470514,12.5325061279 H,0,9.0753990624,17.1593239903,13.1946478535 C,0,5.3018231427,16.6168294605,17.8465598611 C,0,6.5268346139,17.525939974,18.063276384 H,0,6.8040814634,17.4936121968,19.1199822825 H,0,7.3383774068, 17.1021797587,17.468312571 C,0,3.4528955843,19.81071611,18.7887647128 C,0,3.492695464,18.6689464305,19.6074912294 H,0,3.2066107778,17.6999787144,19.2132959166 C,0,3.9430733114,18.75797027,20.9254323927 H,0,3.9734377752,17.8622837818,21.5393189693 C,0,4.3649245495,19.9841540769,21.4444282408 $\mathrm{H}, 0,4.7197637456,20.0504664822,22.4691868948$ C,0,4.3340641507,21.1238900318,20.637658584 H,0,4.6615065235,22.0819271959,21.0328037413 C,0,3.8854179137,21.0382863515,19.3179340677 H,0,3.8694420524,21.9299232594,18.6984543887 C,0,2.3532859495,21.2207792059,16.4988872003 C,0,2.6675772074,21.8013504671,15.2633311384 H,0,3.4023143473,21.3372733986, 14.617939909 C,0,2.031008068,22.9714788365,14.8429530874 
$\mathrm{H}, 0,2.2827372322,23.3957136681,13.875236096$ C,0,1.0762854302,23.5782839019,15.6584185602 $\mathrm{H}, 0,0.5813473651,24.4900649363,15.3349159636$ C,0,0.7549589825,23.0089833026,16.8945554056 $\mathrm{H}, 0,0.0121371884,23.4780682128,17.5342623282$ C, $0,1.3844456733,21.8367108588,17.3096016612$ H,0,1.1311849474,21.4044942716,18.2741007014 C,0,4.3013945858,16.6790078833,13.1679081711 C,0,4.6372816179,15.5249268831,13.8977059388 H,0,4.524888981,15.5208317845,14.9786337579 C,0,5.1512253393,14.4081652399,13.2409719677 $\mathrm{H}, 0,5.4087897941,13.5198837558,13.8116877847$ C, $0,5.3602468728,14.4380249813,11.8586229484$ H,0,5.7780364541,13.5718326642,11.3530711286 C,0,5.042183659,15.5865168866,11.1338132827 H,0,5.2080015683,15.6184566438,10.0602464669 C,0,4.5098843088,16.7036148406,11.7840727971 H,0,4.2678571007,17.5927559093,11.2106048517 C,0,4.4044038268,20.1421608515,12.403528677 $\mathrm{H}, 0,5.4042001838,19.8734856324,12.7395851591$ C,0,4.2226787487,21.2164683126,11.5330822868 H,0,5.086687241,21.7794602551,11.1898882063 C,0,2.9386006608,21.5697119383,11.1064903505 $\mathrm{H}, 0,2.7987508659,22.4138599529,10.4363265446$ C, $0,1.8375977885,20.828114875,11.5364922491$ H,0,0.8374923508,21.0918833937,11.2030004781 C,0,2.0150625222,19.743969068,12.3995232942 H,0,1.145359102,19.1809996963,12.7216088394 C,0,3.295487195,19.3997130081,12.851812384 C, $0,1.9571764162,17.417835901,14.5970835988$ $\mathrm{H}, 0,1.4103912907,17.1414143656,13.6880034837$ H,0,2.2008563826,16.4839329086,15.1165945154 C,0,1.0848428019,18.2969007584,15.5132764558 $\mathrm{H}, 0,0.104990086,17.8102529805,15.6000265599$ $\mathrm{H}, 0,0.8973458547,19.2745245759,15.0518660369$ C,0,1.6390863761,18.4904514942,16.9370201528 H,0,1.961769614,17.5272766935,17.3446450852 H, $0,0.8648508155,18.9053753427,17.5920818011$ $\mathrm{N}, 0,8.2804258268,15.5395404121,14.2718293689$ $\mathrm{H}, 0,7.3573475343,15.1957593784,14.5312641555$ H,0,8.7227699685,15.7931753909,15.1549047367 N,0,6.2916092501,18.914994362,17.5874644715 H,0,7.135187129,19.3539363828,17.2104277294 H,0,5.9484467344,19.4975324495,18.3495095025
O,0,6.5999521737,17.5979719489,15.1316750556 O,0,7.2738537909,19.0008872839,13.5012531759 O,0,4.4671550662,16.9687916889,16.8917605277 O,0,5.1575685906,15.6143293428,18.5289753966 $\mathrm{P}, 0,3.1194525139,19.6163418578,16.9864462571$ $\mathrm{P}, 0,3.618327026,18.1031846534,14.1137032849$ $\mathrm{Ru}, 0,4.9144880683,18.7243783641,15.9062764953$ H,0,7.6521198546,20.1266445847,14.7535999132 $\mathrm{H}, 0,5.4648951787,20.5772467287,15.274830839$ C,0,6.2963021094,21.3517494225,15.2911166522 H,0,6.2120058263,21.8076125079,14.2924064622 O,0,7.5506902885,20.7734410815,15.5000946175 C,0,5.9976188168,22.3547965242,16.388624379 H,0,5.0142293022,22.8118461407,16.2462719085 H,0,6.0168423888,21.8650570085,17.3676942581 H,0,6.7619191956,23.1387807517,16.38357975

\section{TS(d-II) for Acetone}

C,0,6.9005158229,19.261636799,14.5141994134 C,0,7.3443722489,17.7989189528,14.6851184938 $\mathrm{H}, 0,7.6948219807,17.414609955,13.7243246645$ $\mathrm{H}, 0,8.188906742,17.7911546212,15.3798423175$ C,0,7.4262950958,16.6937758253,18.1076658414 C,0,6.5742287712,17.691450642,18.887611373 H,0,5.7621648441,17.2071576624,19.4256802784 $\mathrm{H}, 0,7.2278236765,18.2189596223,19.5916033552$ C,0,3.0034074812,19.7425156486,18.8158141965 C,0,3.3098771691,18.6236169265,19.6097109002 H,0,3.5985744444,17.6853584566,19.1471149558 C,0,3.2769417446,18.7115916009,21.0022868736 $\mathrm{H}, 0,3.5195553882,17.835469299,21.5978793803$ C,0,2.9454616732,19.9182809424,21.6216568879 $\mathrm{H}, 0,2.9266844504,19.9890208934,22.705928495$ C,0,2.6449921191,21.0365215278,20.8411801391 H,0,2.3911457197,21.9805163255,21.3163334959 C,0,2.6721732031,20.9511546708,19.4478912133 H,0,2.4409892463,21.8294639641,18.8531344833 C,0,3.0025287802,21.3036950305,16.401186565 C,0,4.2318692416,21.9825442654,16.370948247 $\mathrm{H}, 0,5.1433538982,21.4541552135,16.6262993872$ C,0,4.3015191684,23.3068605806,15.9431178309 H,0,5.2636824019,23.8105977149,15.9134617546 C,0,3.144825195,23.9719647283,15.529050714 H,0,3.2003234837,25.0003988182,15.1823154143 
C,0,1.9183814111,23.3078739182,15.5561642228 H,0,1.01421988,23.8168050123,15.2329091018 C,0,1.8458250137,21.9846902026,15.9965365393 H,0,0.8785816592,21.493974483,16.0125439966 C,0,3.9691125737,16.7663579279,12.9712746313 C,0,3.3817742866,15.5021668483,12.8251511289 H,0,2.4983989955,15.2370843255,13.3983998578 C,0,3.9172954387,14.5684601974,11.9341384701 H,0,3.4474379312,13.5940586936,11.8298938841 C,0,5.0483027247,14.885412508,11.181115312 H,0,5.4657946139,14.1573642022,10.4912055418 C,0,5.6385282436,16.1454683555,11.3142255667 H,0,6.514853279,16.4043719923,10.7263508586 C,0,5.1029780525,17.080739316,12.1990764374 H,0,5.5661728088, 18.0610631377,12.2877770533 C, $0,3.9385771507,20.6504037041,13.2905436974$ $\mathrm{H}, 0,4.68875314,20.6860763001,14.0691633753$ C,0,3.736046109,21.7595938121,12.4646466401 $\mathrm{H}, 0,4.3260061908,22.6558173239,12.6316007676$ C,0,2.7877343973,21.71695511,11.4451349691 H,0,2.6292889949,22.5828273596,10.8073344806 C,0,2.0474675811,20.5490179143,11.2345992469 $\mathrm{H}, 0,1.3176334737,20.5010665172,10.4304325168$ C,0,2.2486665795,19.4405450144,12.0531812604 H,0,1.6797212991,18.5345907601,11.8616140024 C,0,3.1850694932,19.4856730671,13.1024784718 C,0,1.6266023086,17.5565209376,14.5337680414 H,0,1.1278257469, 17.3162306505,13.5891459317 $\mathrm{H}, 0,1.6361022146,16.6472230766,15.1414572235$ C,0,0.8449328491,18.671373774,15.2552908942 H,0,-0.2131852029,18.379030604,15.275981581 H,0,0.8973299044,19.5874511515,14.6563448064 C, $0,1.2731152035,18.9558902165,16.7066898534$ H,0,1.2034107433,18.0393278323,17.2990811425 $\mathrm{H}, 0,0.5866740942,19.674756588,17.1685478886$ $\mathrm{N}, 0,6.2703464559,16.9386081894,15.2549974796$ $\mathrm{H}, 0,6.6700581605,16.2789511909,15.9218644134$ $\mathrm{H}, 0,5.8635556192,16.372644632,14.5126956017$ N,0,5.9896960316,18.6376982506,17.9029511892 H,0,6.7450890295,19.1204618735,17.4102139634 H,0,5.4964201823,19.3568019107,18.4302510419 O,0,5.9780179556,19.6766651619,15.3468154356 O,0,7.4465072249,19.9630880998,13.6733560527 O,0,6.9049591967,15.4942018984,17.891667557
O,0,8.4939805054,17.0424785073,17.6338080775 P,0,3.0214869217,19.5501497748,16.9696396662 $\mathrm{P}, 0,3.4019748964,18.0164918736,14.2087876899$ $\mathrm{Ru}, 0,4.6645172481,18.1847446036,16.1191213461$ H,0,3.8396824669,16.8064192366,16.8342238862 H,0,5.9092338882,15.4433038869,18.2254649105 C,0,3.7213093791,15.5330943092,17.5143476649 O,0,4.4768849383,15.5368220569,18.5576765179 C,0,4.1003397414,14.5920789018,16.3685266923 $\mathrm{H}, 0,3.5463064642,14.8110436803,15.4539621022$ $\mathrm{H}, 0,5.1700238373,14.6354706236,16.1597677296$ H,0,3.8657776504,13.5679010176,16.6876166104 C,0,2.2216648007,15.5460008878,17.8145906278 $\mathrm{H}, 0,1.6091031878,15.6800646549,16.9196901071$ H,0,1.9747451945,14.5732162146,18.2586995859 $\mathrm{H}, 0,1.9784541295,16.3177823622,18.54722591$ 


\section{References}

(1) L. N. Ding, A. Q. Wang, M. Y. Zheng and T. Zhang, ChemSusChem, 2010, 3, 818-821.

(2) (a) Becke, A.D., J. Chem. Phys. 1993, 98, 5648. (b) Lee, C.; Yang, W.; Parr, R.G., Phys. Rev. $B$ 1988, 37, 785.

(3) Kaupp, M.; Schleyer, P.V.R.; Stoll, H.; Preuss, H., J. Chem. Phys. 1991, 94, 1360.

(4) Grimme, S.; Antony, J.; Ehrlich, S.; Krieg, H., J. Chem. Phys. 2010, 132, 154104.

(5) Zhao, Y.; Truhlar, D. G., Theor. Chem. Acc. 2008, 120, 215.

(6) Marenich, A. V.; Cramer, C. J.; Truhlar, D. G., J. Phys. Chem. B 2009, 113, 6378.

(7) Gaussian 09, Revision D.01, Frisch, M. J.; Trucks, G. W.; Schlegel, H. B.; Scuseria, G. E.; Robb, M. A.; Cheeseman, J. R.; Scalmani, G.; Barone, V.; Mennucci, B.; Petersson, G. A.; Nakatsuji, H.; Caricato, M.; Li, X.; Hratchian, H. P.; Izmaylov, A. F.; Bloino, J.; Zheng, G.; Sonnenberg, J. L.; Hada, M.; Ehara, M.; Toyota, K.; Fukuda, R.; Hasegawa, J.; Ishida, M.; Nakajima, T.; Honda, Y.; Kitao, O.; Nakai, H.; Vreven, T.; Montgomery, J. A., Jr.; Peralta, J. E.; Ogliaro, F.; Bearpark, M.; Heyd, J. J.; Brothers, E.; Kudin, K. N.; Staroverov, V. N.; Kobayashi, R.; Normand, J.; Raghavachari, K.; Rendell, A.; Burant, J. C.; Iyengar, S. S.; Tomasi, J.; Cossi, M.; Rega, N.; Millam, J. M.; Klene, M.; Knox, J. E.; Cross, J. B.; Bakken, V.; Adamo, C.; Jaramillo, J.; Gomperts, R.; Stratmann, R. E.; Yazyev, O.; Austin, A. J.; Cammi, R.; Pomelli, C.; Ochterski, J. W.; Martin, R. L.; Morokuma, K.; Zakrzewski, V. G.; Voth, G. A.; Salvador, P.; Dannenberg, J. J.; Dapprich, S.; Daniels, A. D.; Farkas, Ö.; Foresman, J. B.; Ortiz, J. V.; Cioslowski, J.; Fox, D. J. Gaussian, Inc., Wallingford CT, 2013. 


\section{NMR spectra}

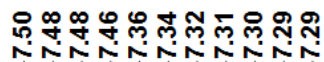

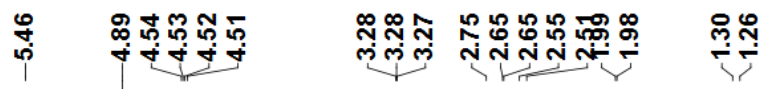

$1 \mathrm{H}$ of Ru1

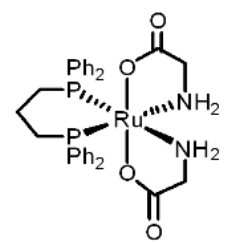

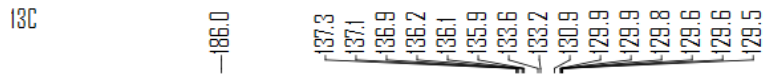

13 C of Ru1
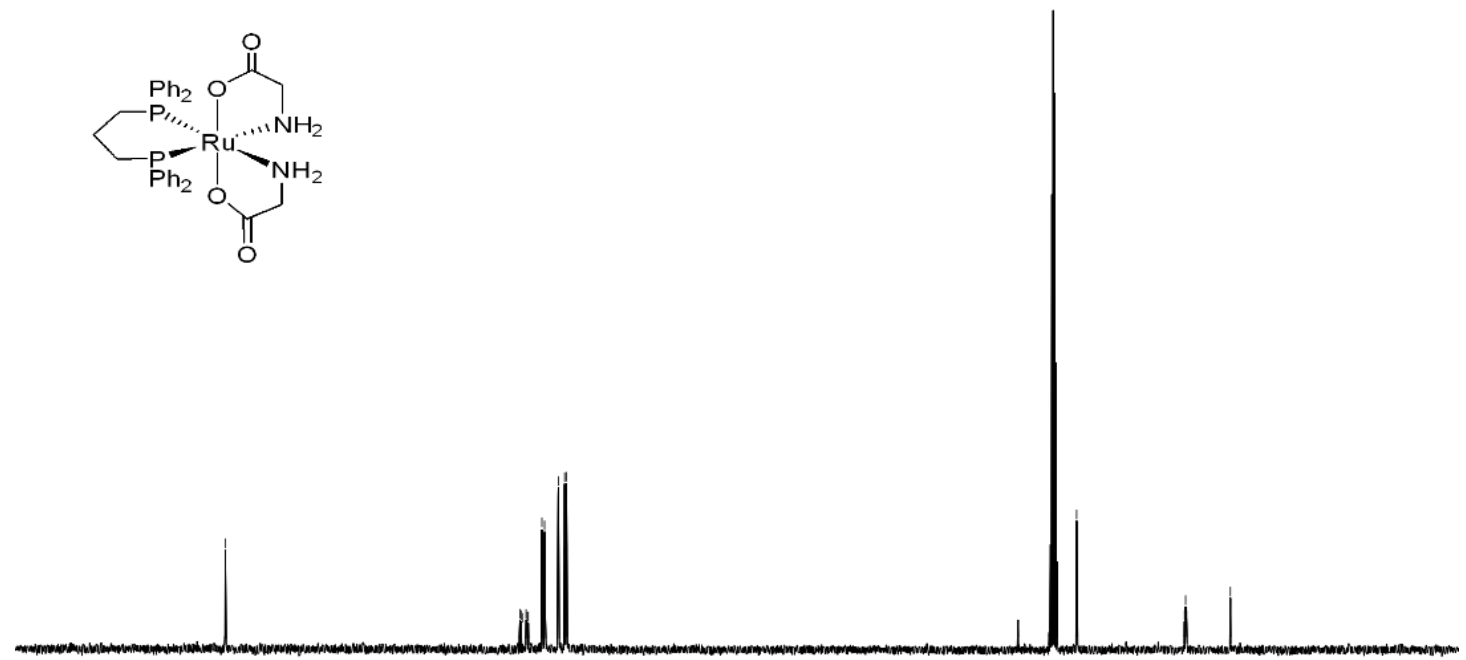

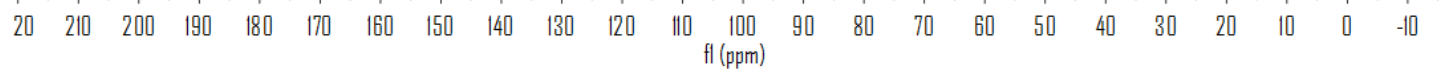




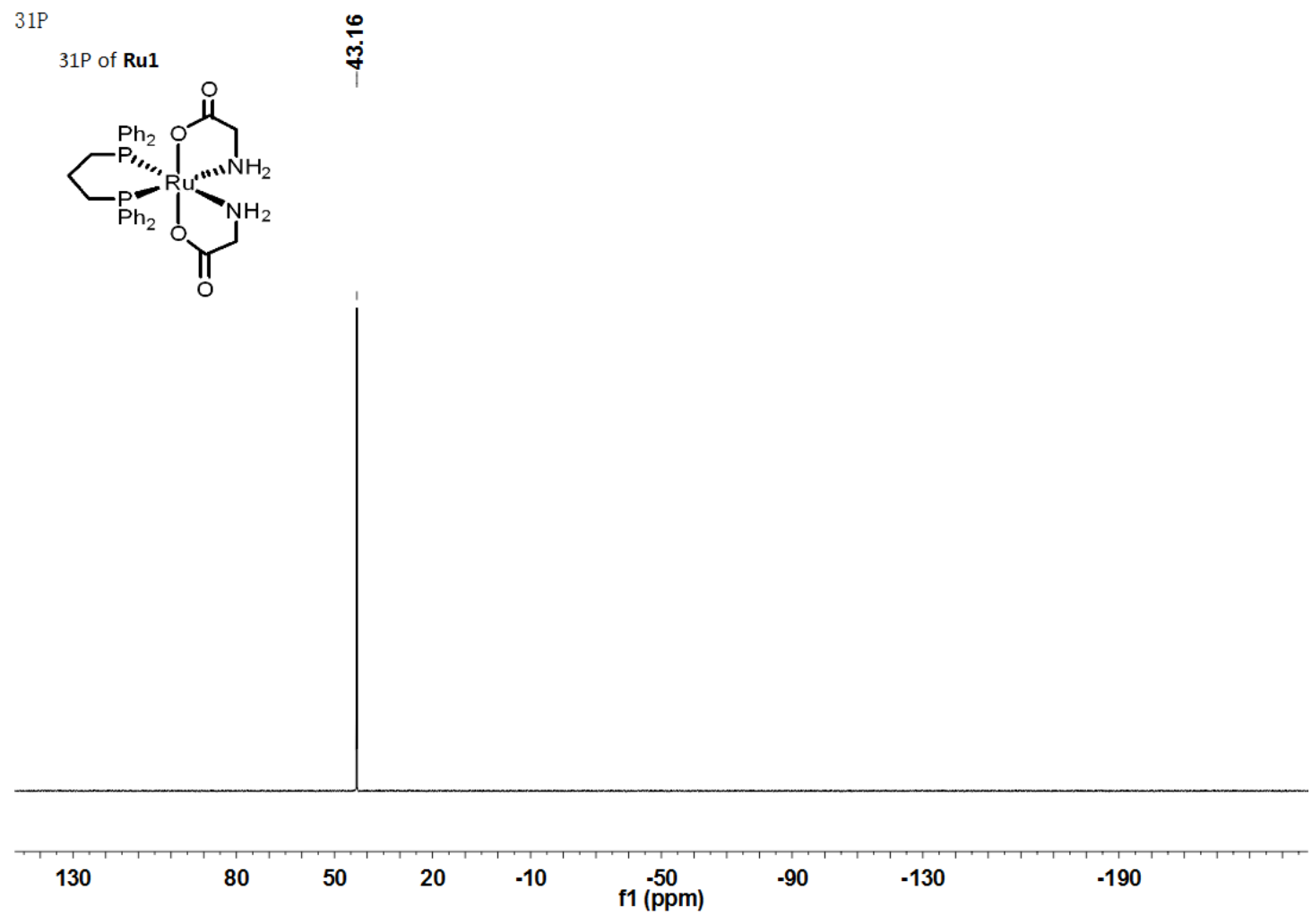

H

S1 product's $1 \mathrm{H}$<smiles>OCc1ccccc1</smiles>

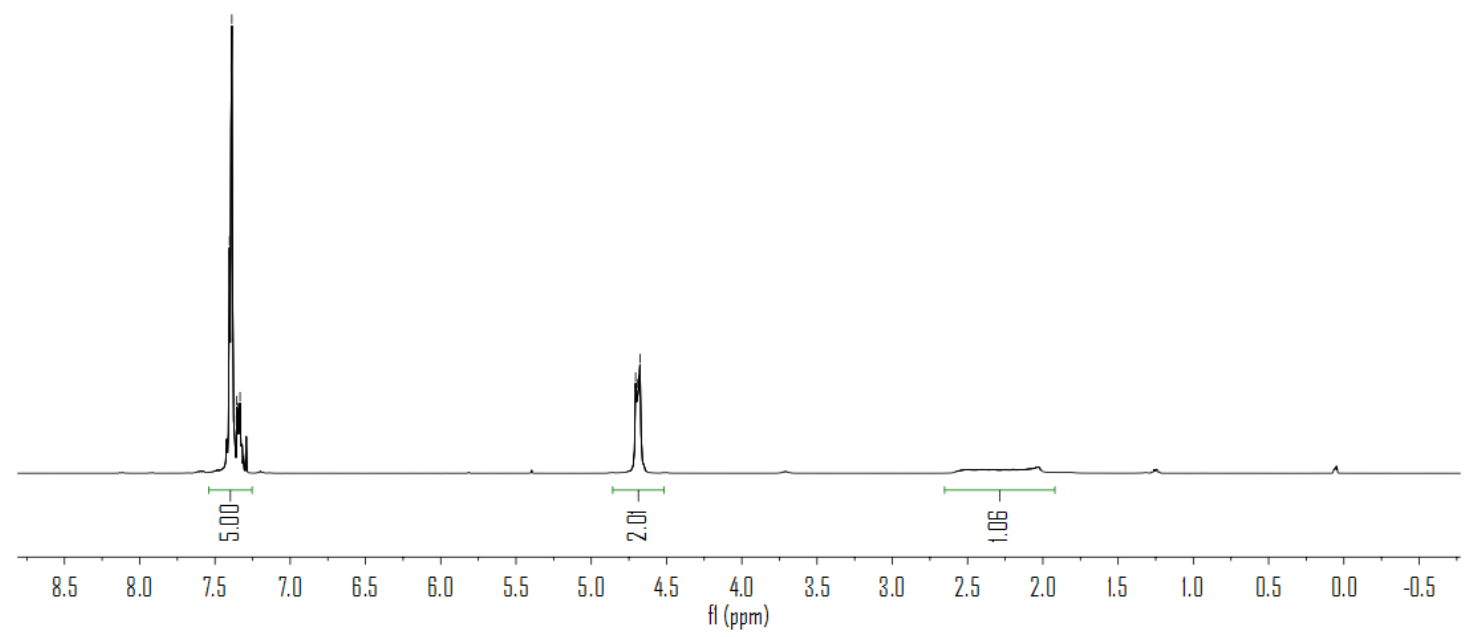


S1 product' $13 C$

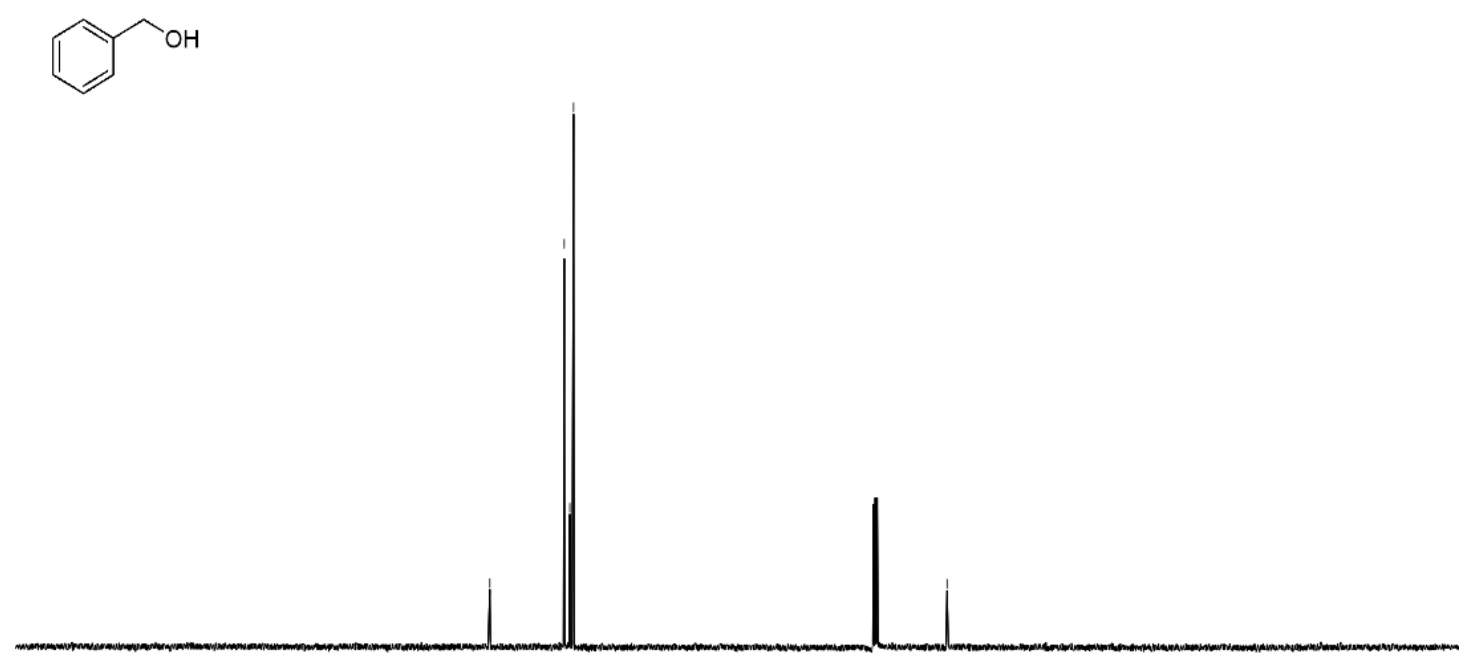

$\begin{array}{lllllllllllllllllllllll}210 & 200 & 190 & 180 & 170 & 160 & 150 & 140 & 130 & 120 & 110 & \begin{array}{c}100 \\ \mathrm{fl}(\mathrm{ppm})\end{array} & 90 & 80 & 70 & 60 & 50 & 40 & 30 & 20 & 10 & 0 & -10\end{array}$

IH

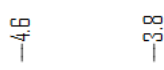

S2 product's $1 \mathrm{H}$<smiles>COc1ccc(CO)cc1</smiles>

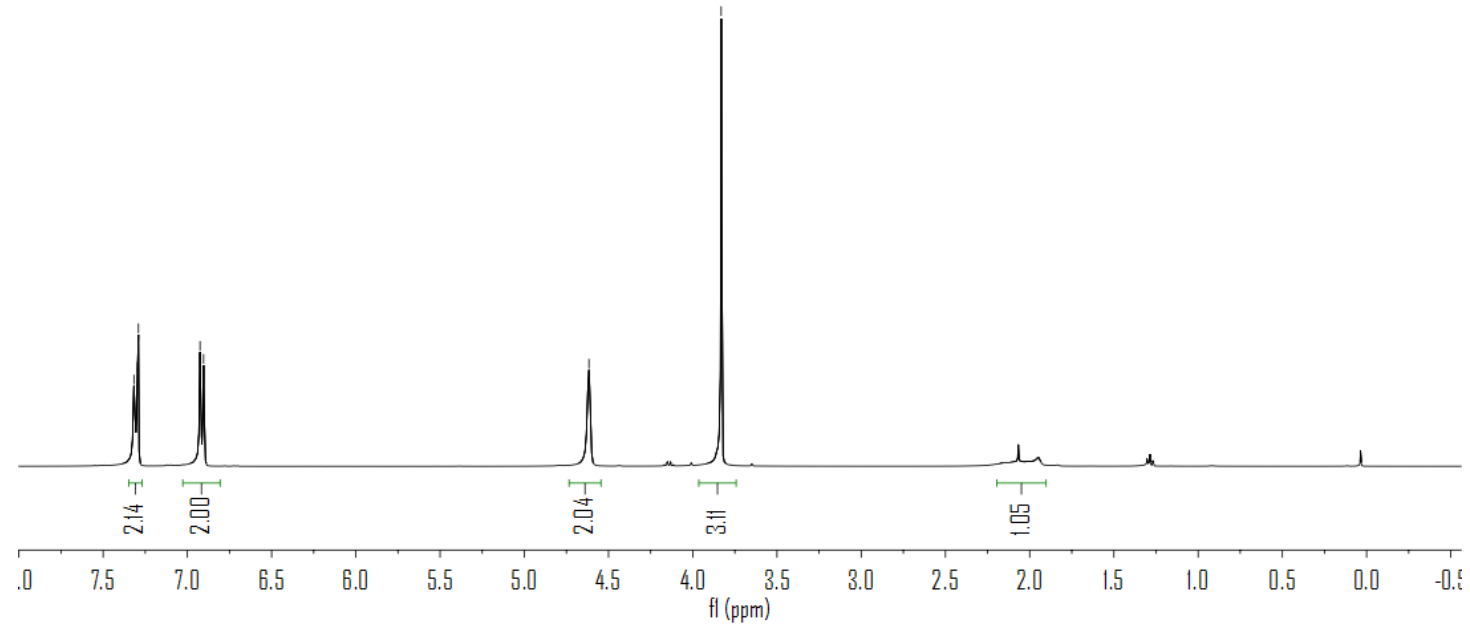


S2 product's $13 C$<smiles>COc1ccc(CO)cc1</smiles>

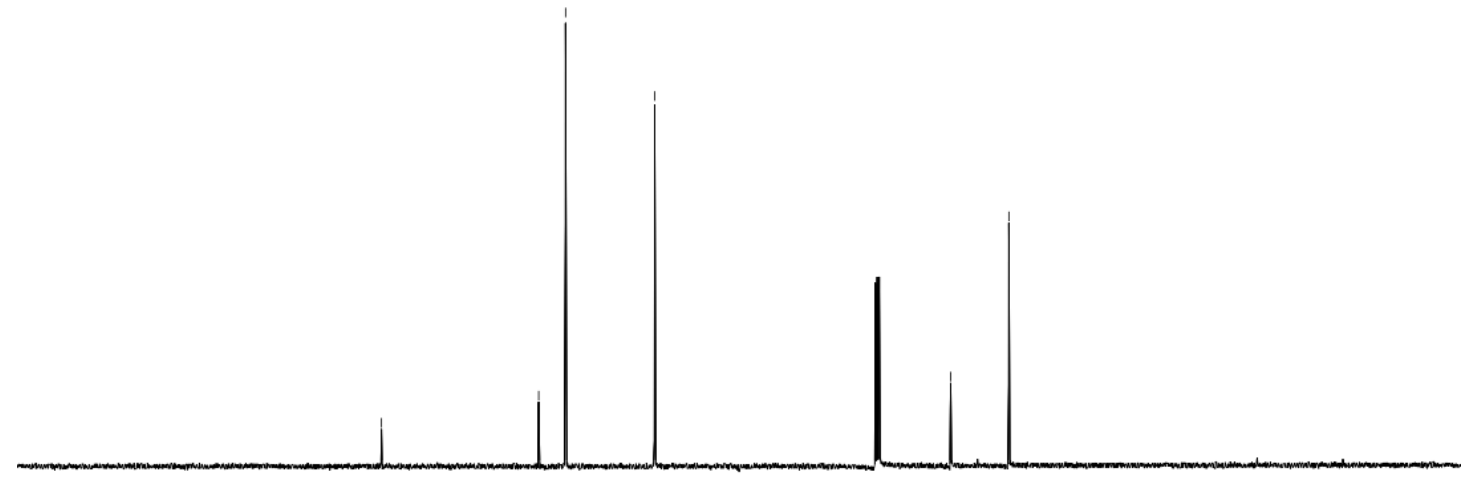

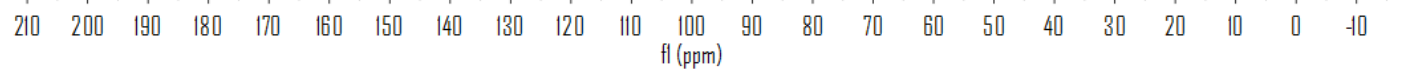

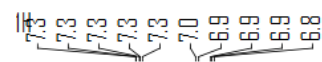

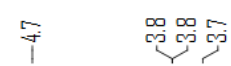

ָ

S3 product's $1 \mathrm{H}$<smiles>COc1cccc(CO)c1</smiles>

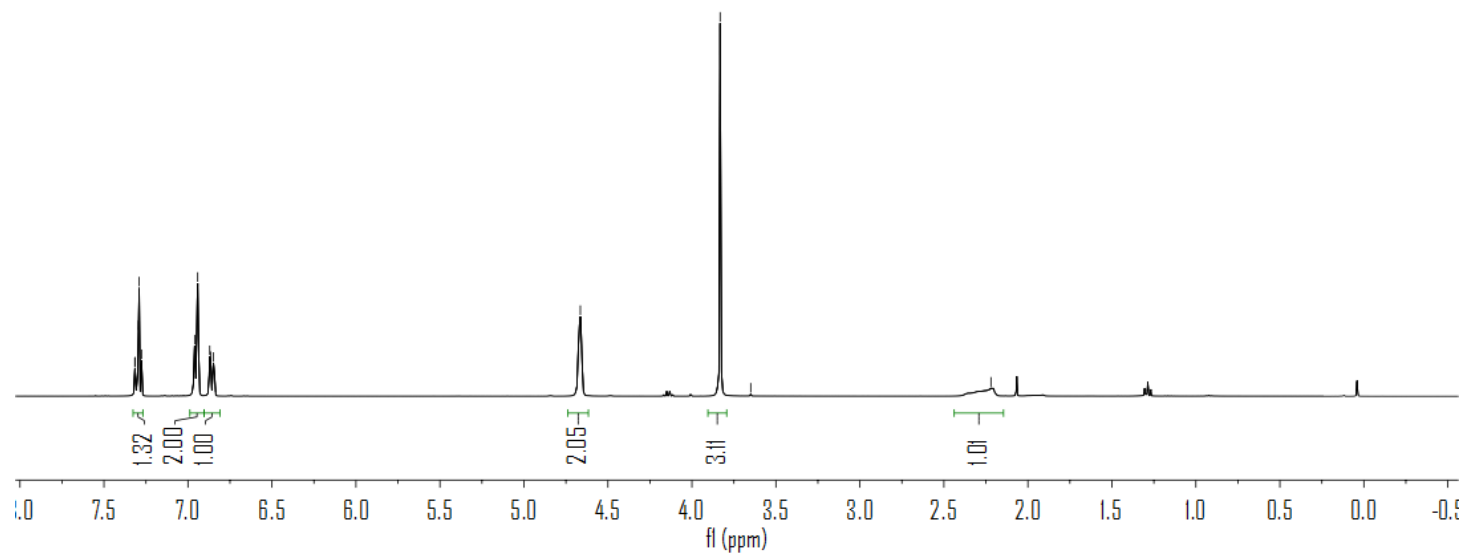


S3 product's $13 C$

$\mathrm{MeO} \mathrm{OH}$

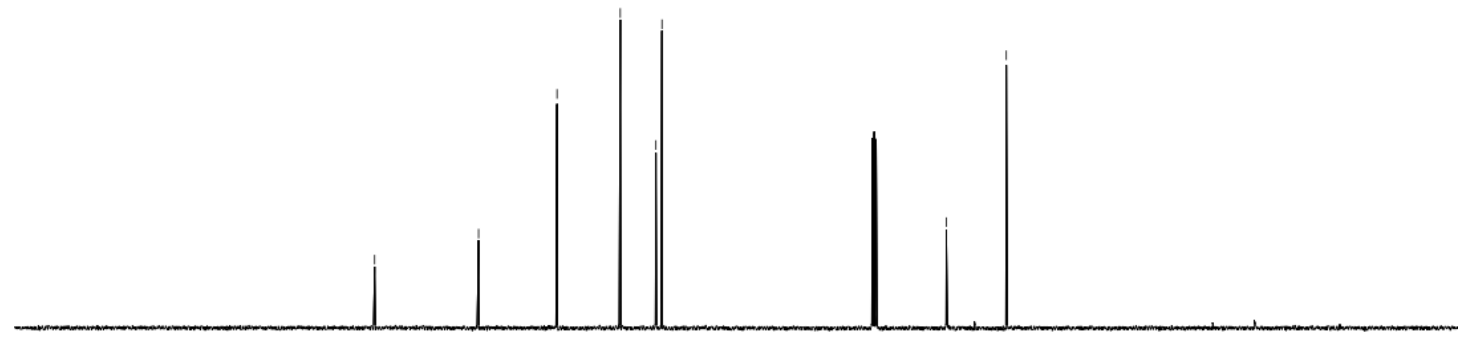

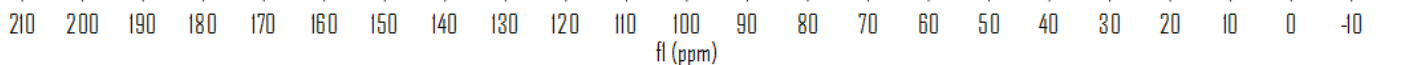

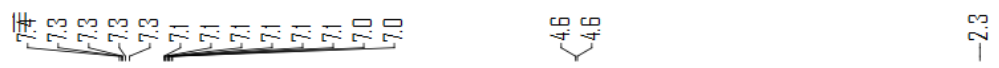

S4 product's $1 \mathrm{H}$

OH

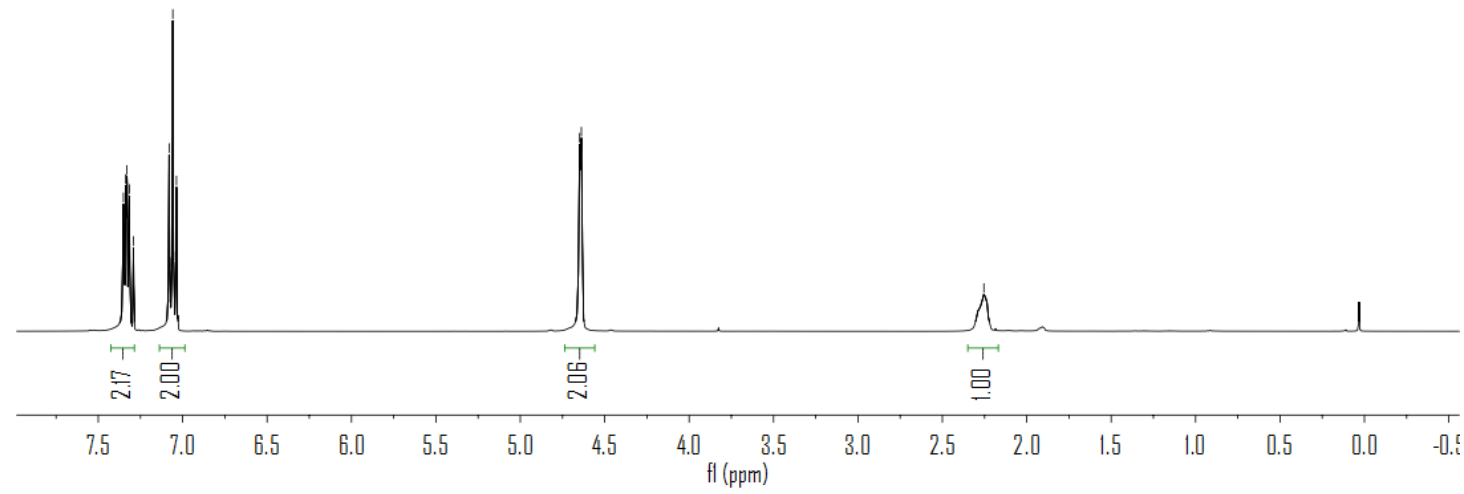


S4 product's $13 \mathrm{C}$<smiles>OCc1ccc(F)cc1</smiles>

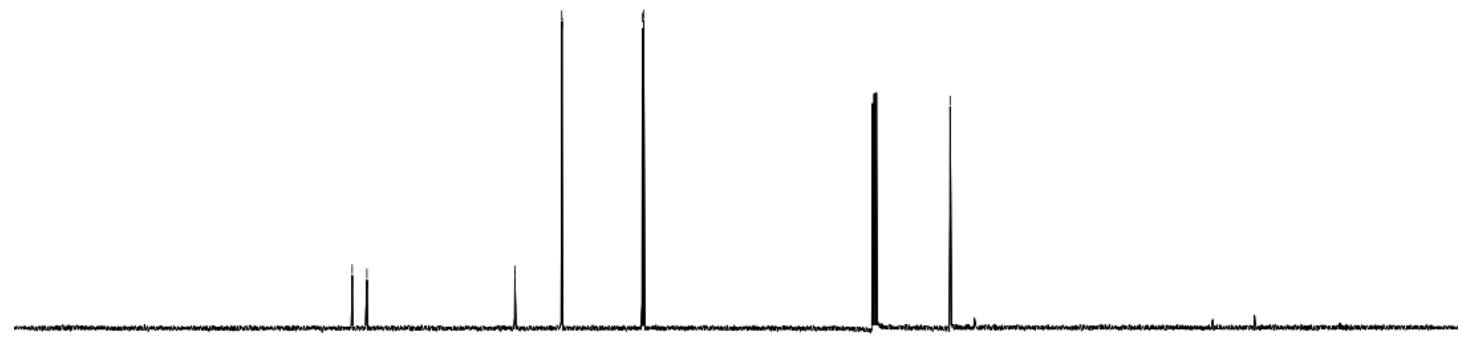

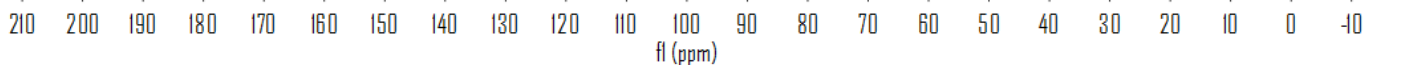

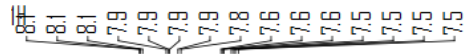

ज्ञ

S5 product's $1 \mathrm{H}$<smiles>OCc1cccc2ccccc12</smiles>

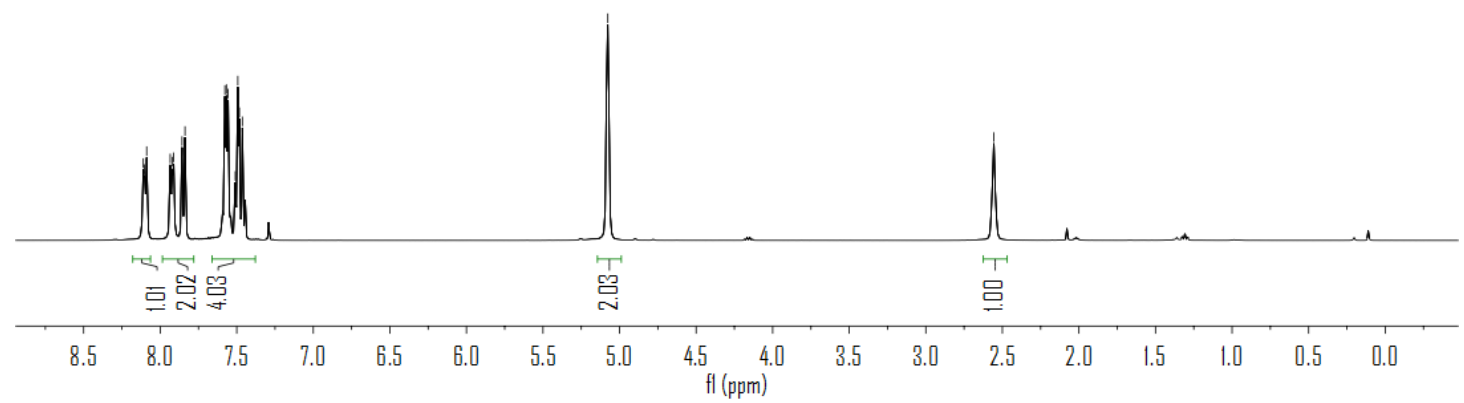


S5 product's $13 \mathrm{C}$<smiles>OCc1cccc2ccccc12</smiles>

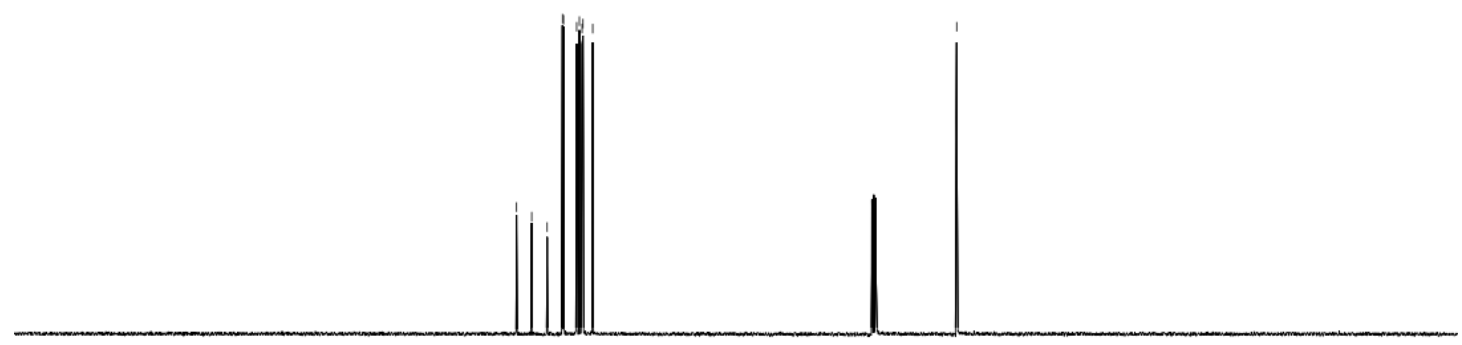

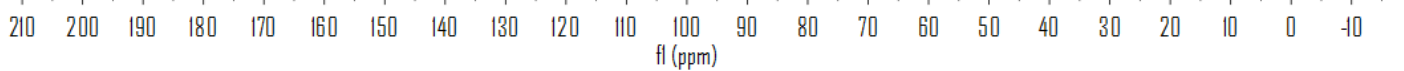

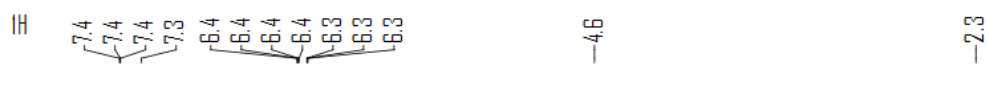

S6 product's $1 \mathrm{H}$<smiles>OCc1ccco1</smiles>

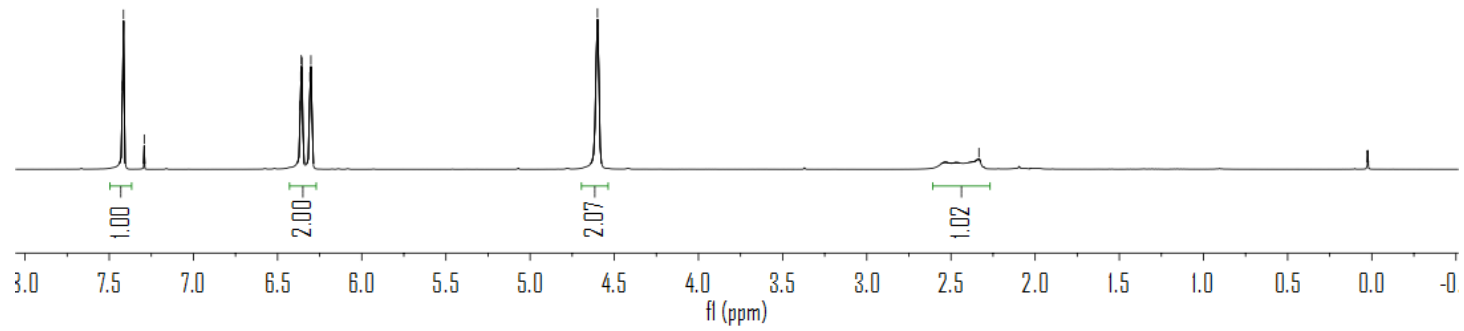


S6 product's $13 C$<smiles>OCc1ccco1</smiles>

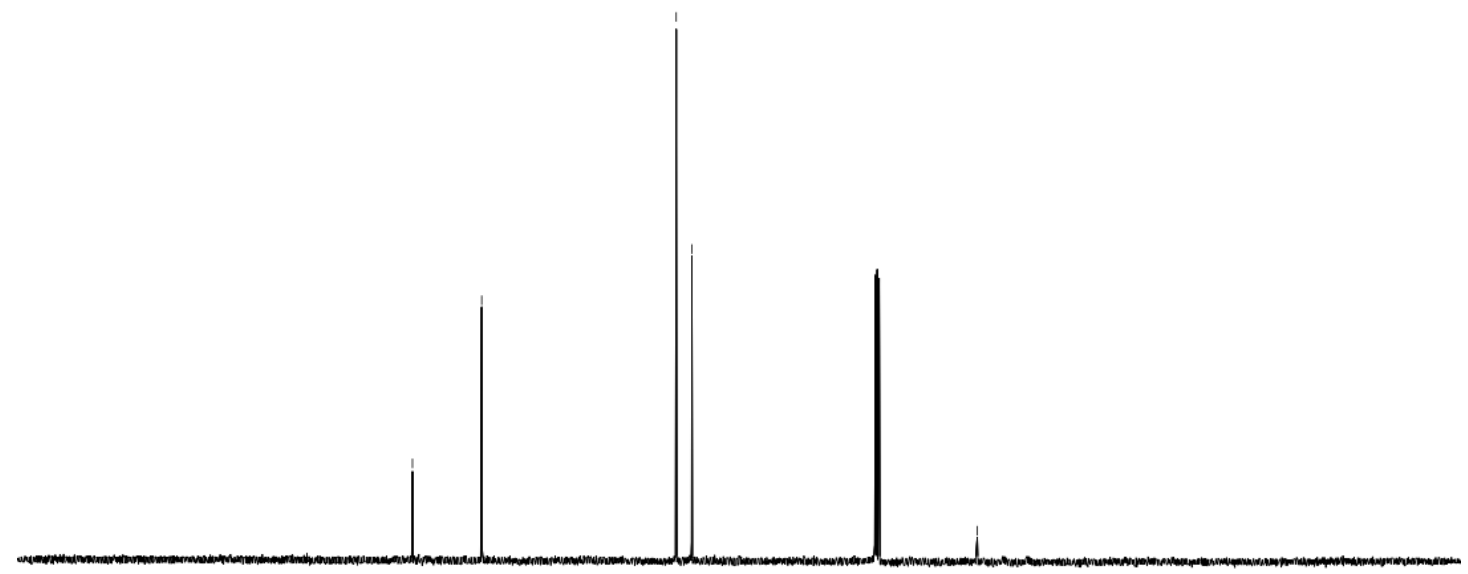

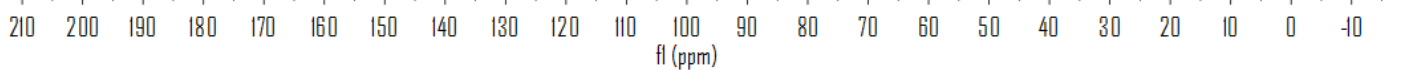

S7 product's $1 \mathrm{H}$

$\sim \sim^{\circ H}$

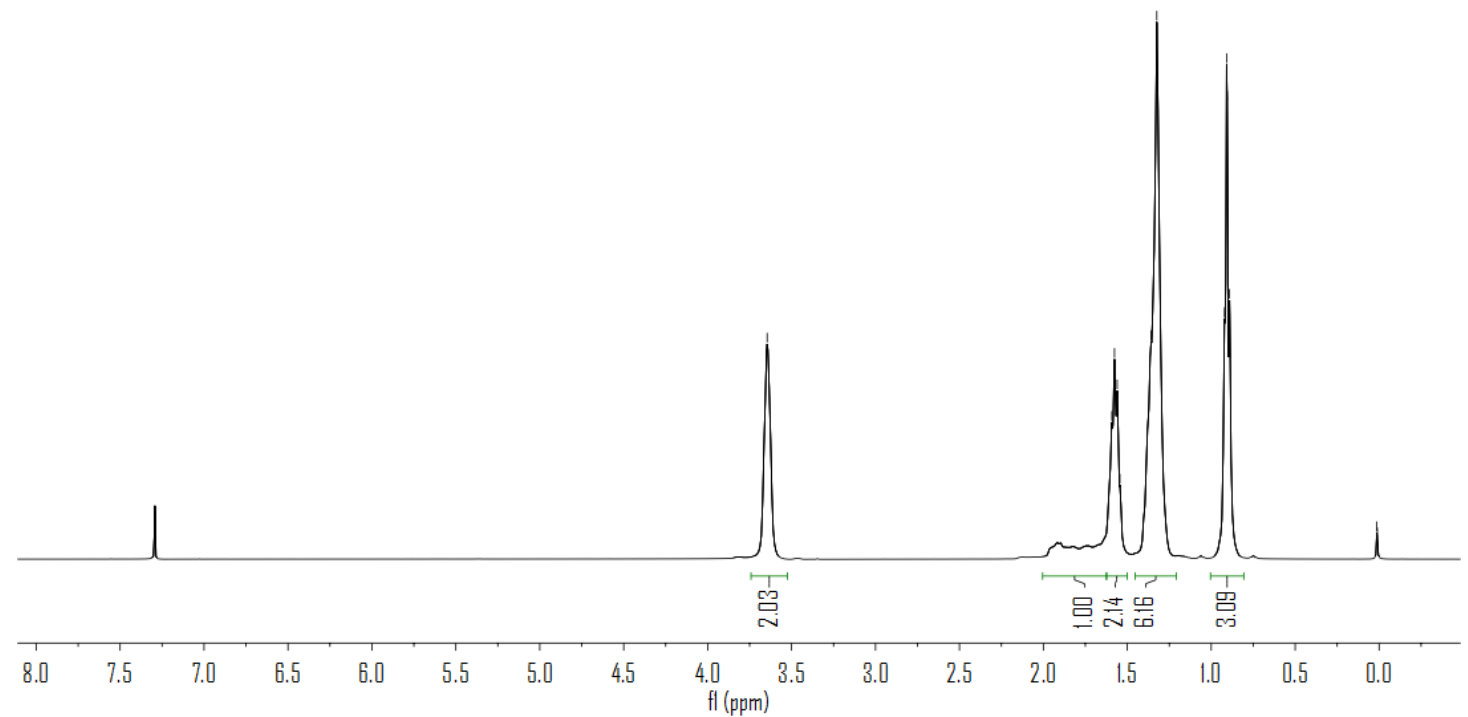


S7 product's $13 C$

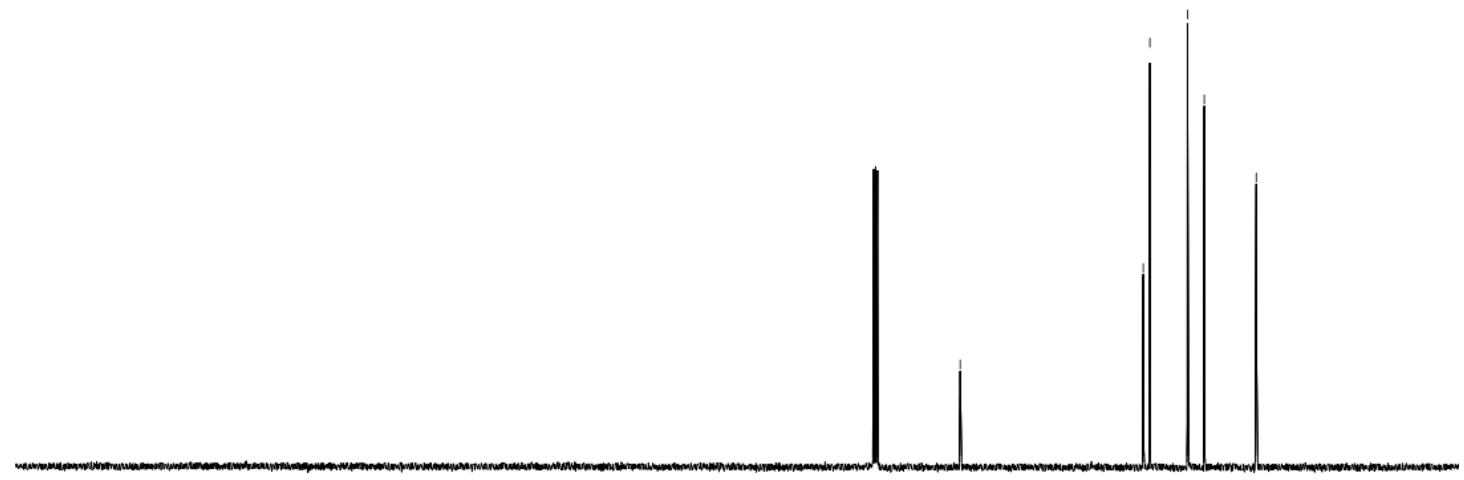

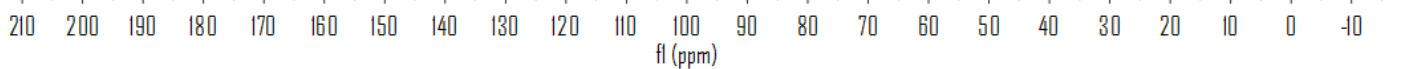
H

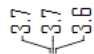

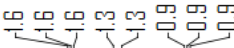

S8 product's $1 \mathrm{H}$

$\curvearrowright \curvearrowright \mathrm{NOH}$

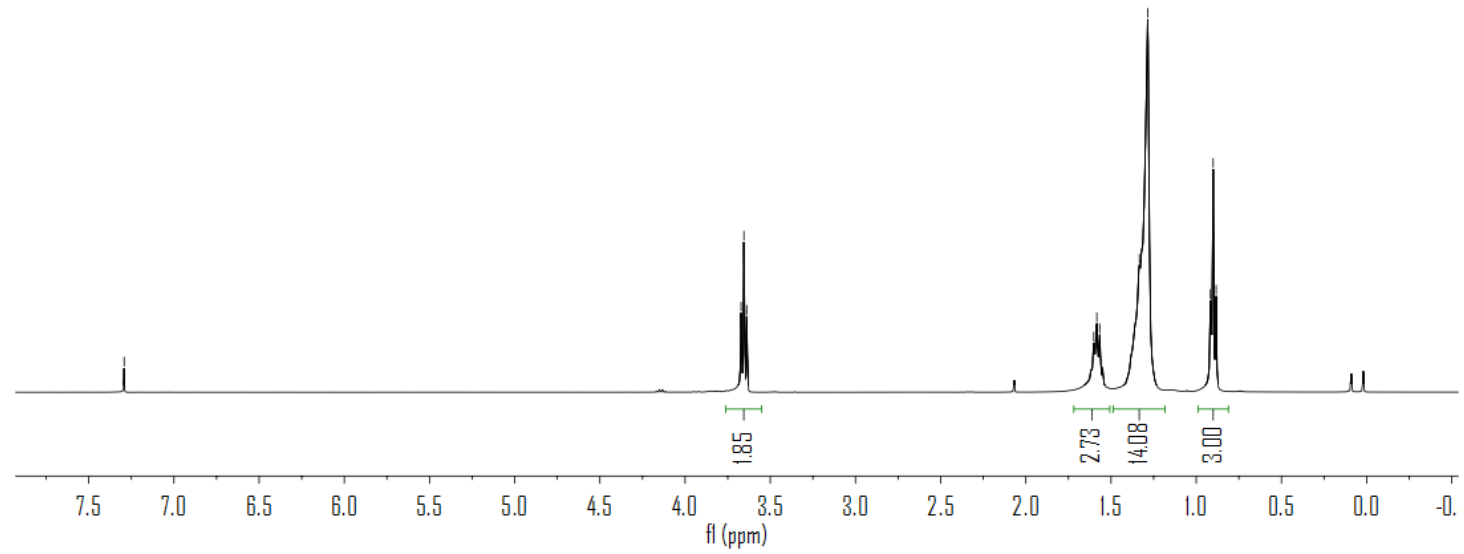


S8 product's $13 \mathrm{C}$

$\sim^{\mathrm{OH}}$

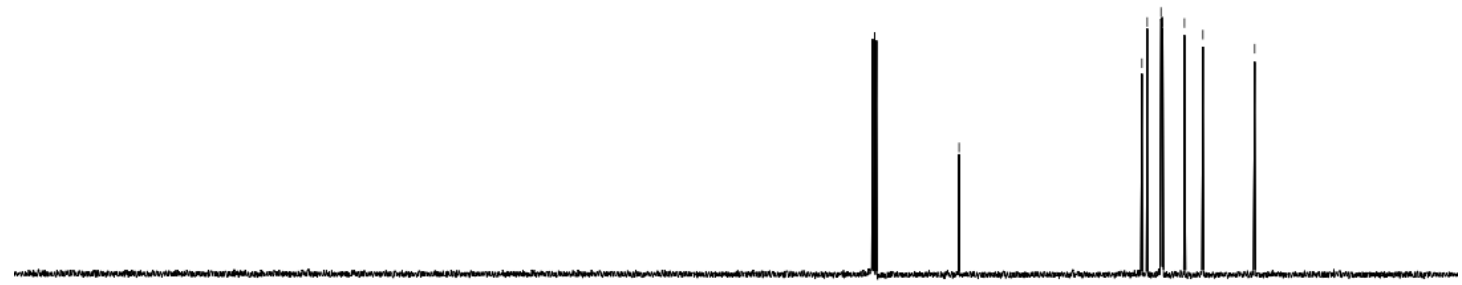

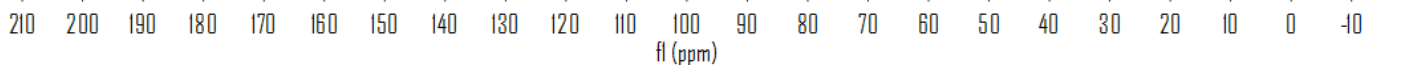

H

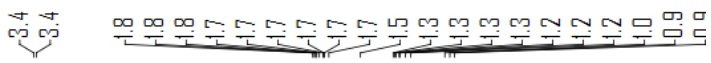

S9 product's $1 \mathrm{H}$<smiles>OCC1CCCCC1</smiles>

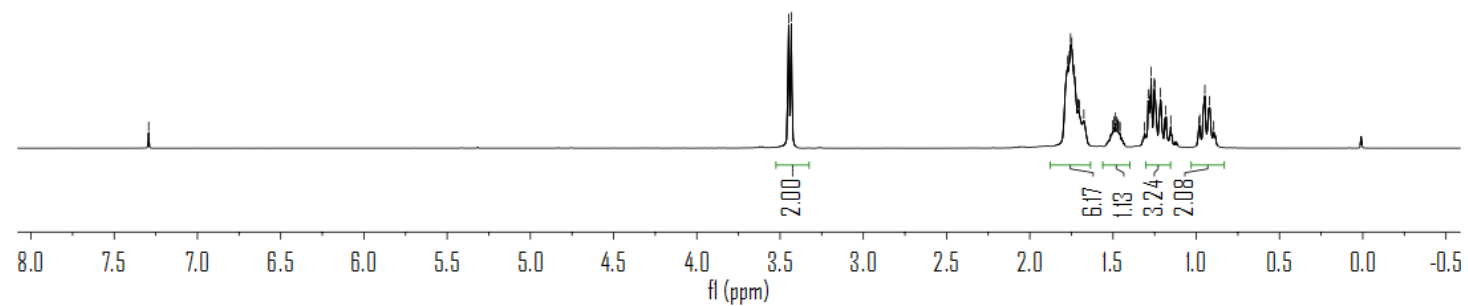


S9 product's $13 \mathrm{C}$

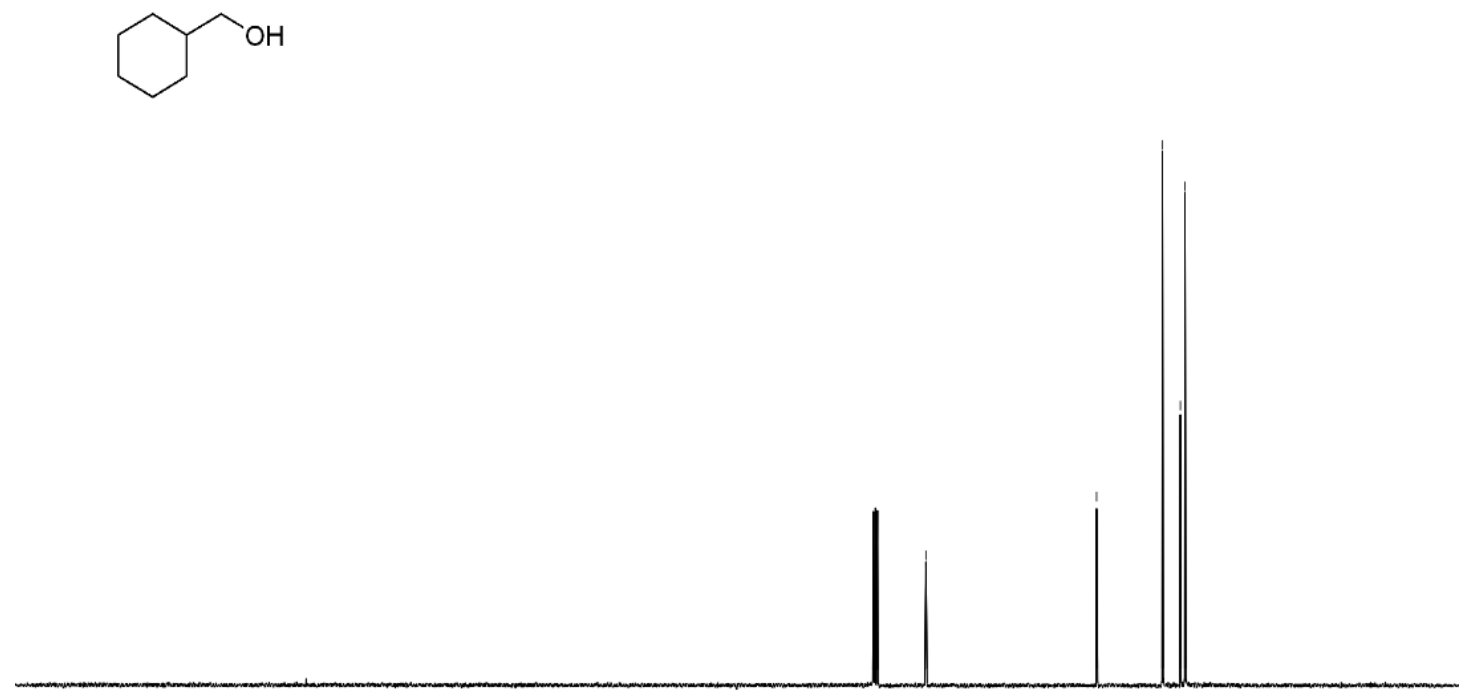

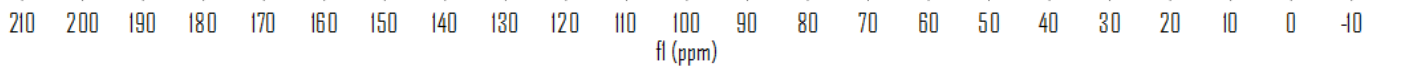

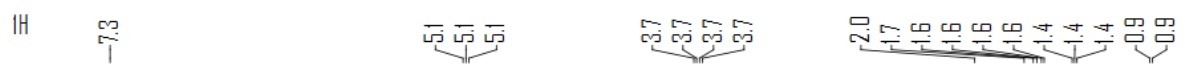

S10 product's $1 \mathrm{H}$

$\mathrm{N}_{\mathrm{OH}}$

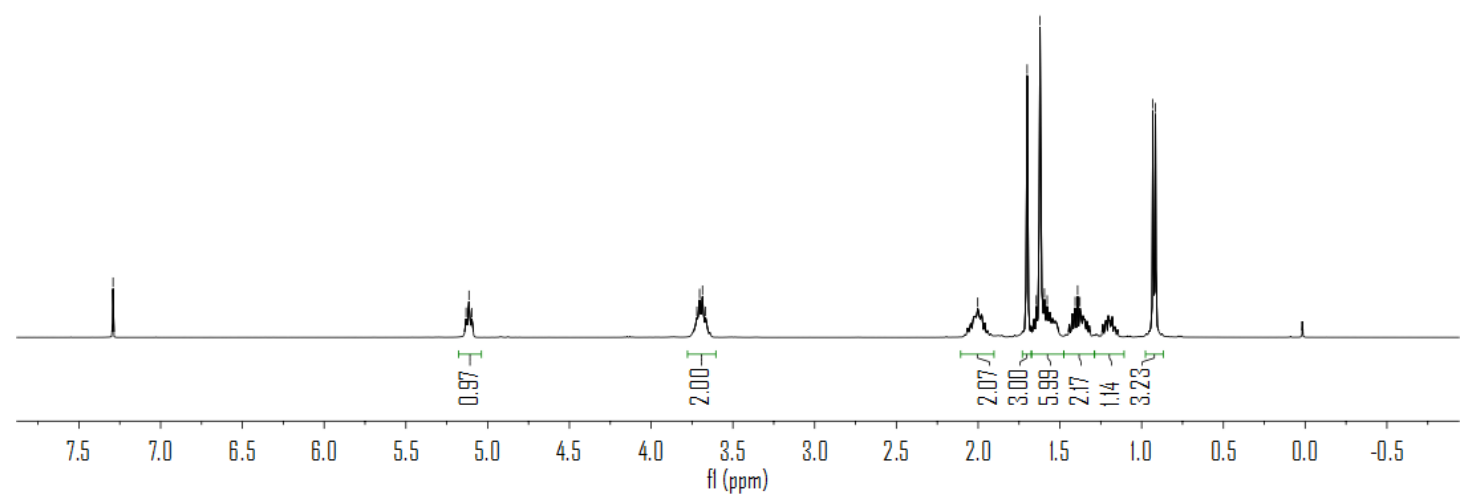


S10 product's $13 \mathrm{C}$

$\mathrm{N}_{\mathrm{OH}}$

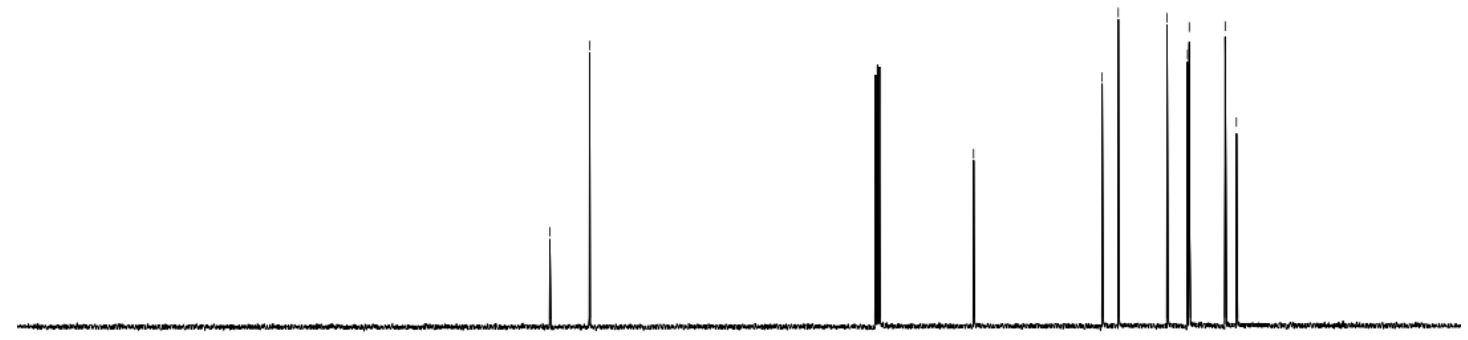

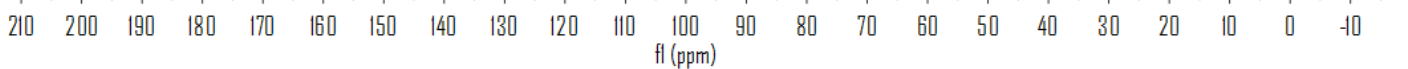




\section{GC, HPLC chromatography}

<Sample Information>

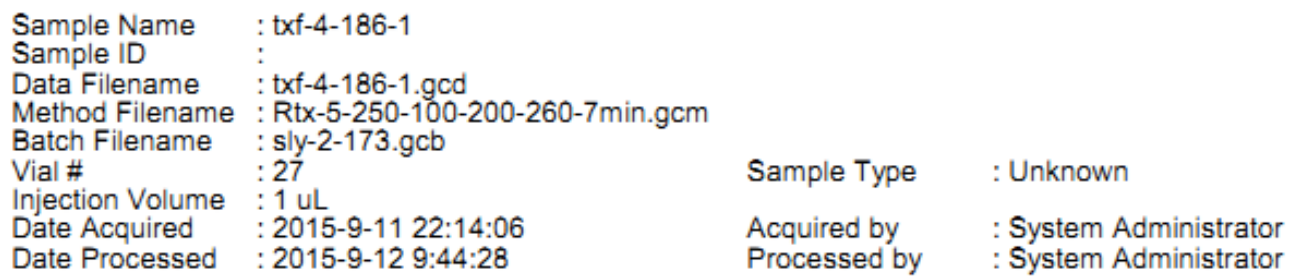

\section{$<$ Chromatogram>}

uV

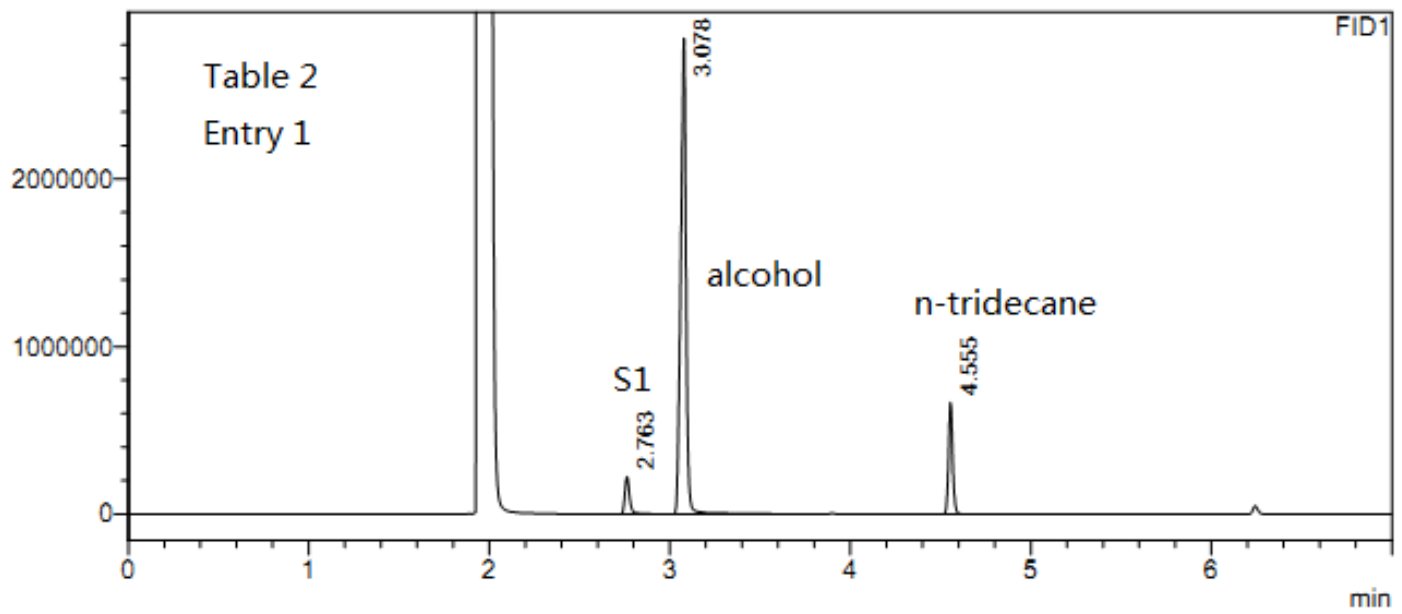

<Peak Table>

FID1

\begin{tabular}{|r|r|r|r|r|r|r|r|}
\hline Peak\# & Ret. Time & \multicolumn{1}{|c|}{ Area } & \multicolumn{1}{c|}{ Height } & \multicolumn{1}{c|}{ Conc. } & Unit & Mark & Name \\
\hline 1 & 2.763 & 404061 & 217633 & 5.594 & & $\mathrm{M}$ & \\
\hline 2 & 3.078 & 5764715 & 2812830 & 79.815 & & $\mathrm{M}$ & \\
\hline 3 & 4.555 & 1053796 & 642428 & 14.590 & & $\mathrm{M}$ & \\
\hline Total & & 7222572 & 3672891 & & & & \\
\hline
\end{tabular}




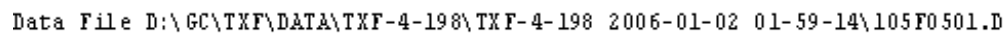
Sample Iame: TXF-4-198-3

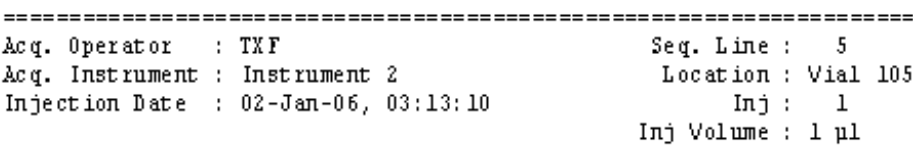

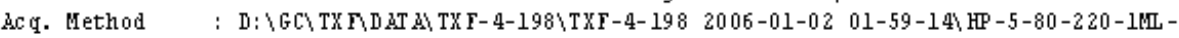
12MIII.M

Last changed : $1 / 14 / 2016$ 6:10:43 PM by TX F

Aralys is Method : D: \GC TX NMETHOD HP-5-80-200-1ML-15MIM.M

Last charged : $1 / 2 / 2006$ 7:49:42 PM by TXY

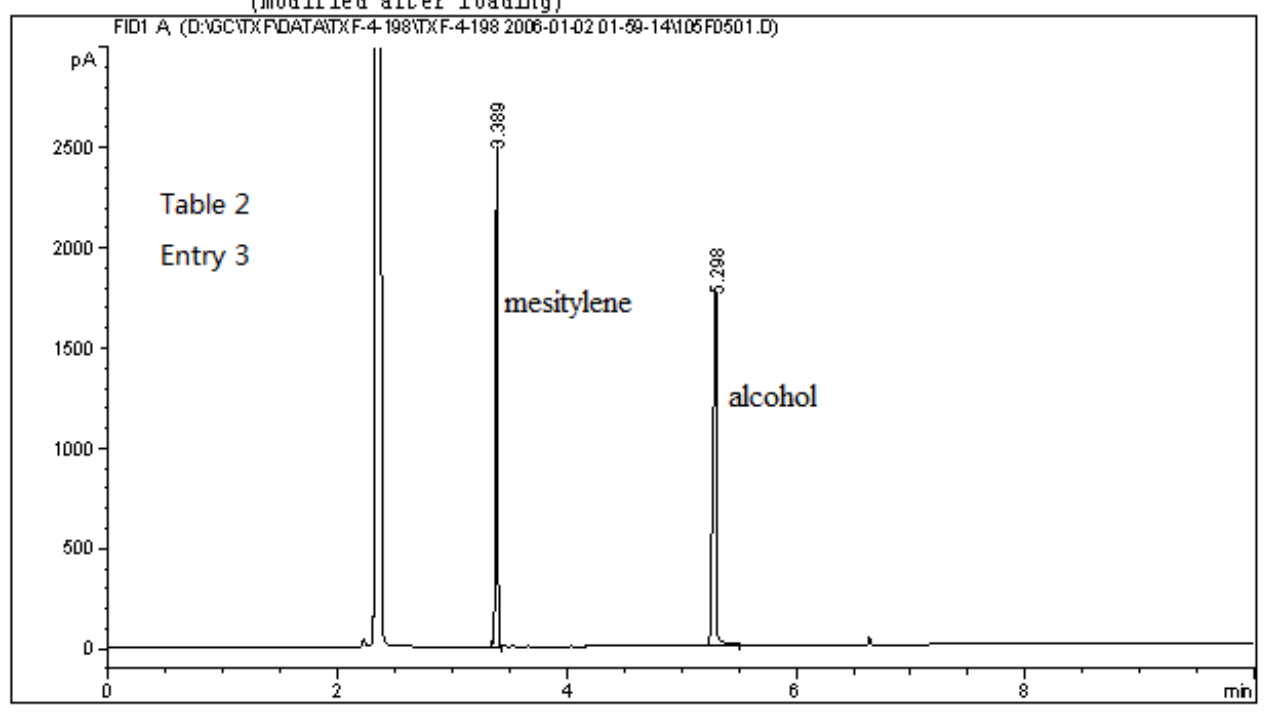

Area Percent Repor

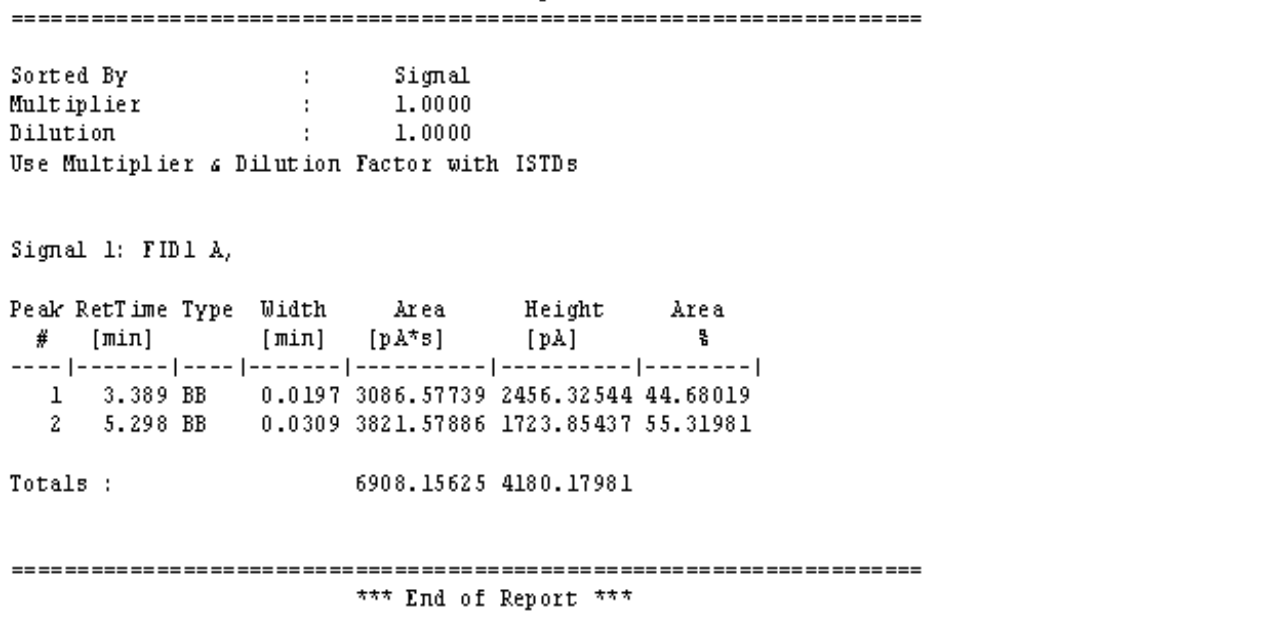




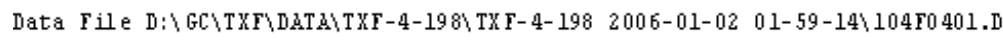
Sample Ilame: TXr-4-200-2

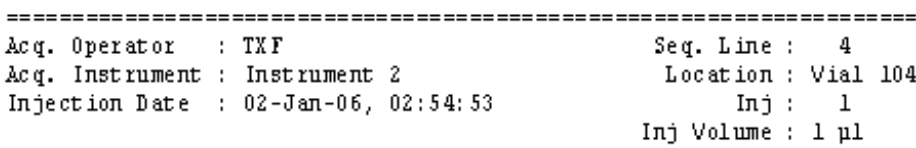

Acq. Method : D : $\backslash$ GC TX $\backslash D$ AT A TX F-4-198\TXF-4-198 2006-01-02 01-59-14\ HP-5-80-220-1ML-

Last charged: : $1 / 14 / 2016 \quad 6: 10: 43$ PM by TXF

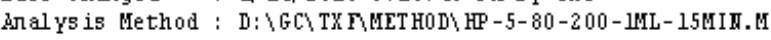

Last charged : $1 / 2 / 2006$ 7:49:42 PM bY TXP

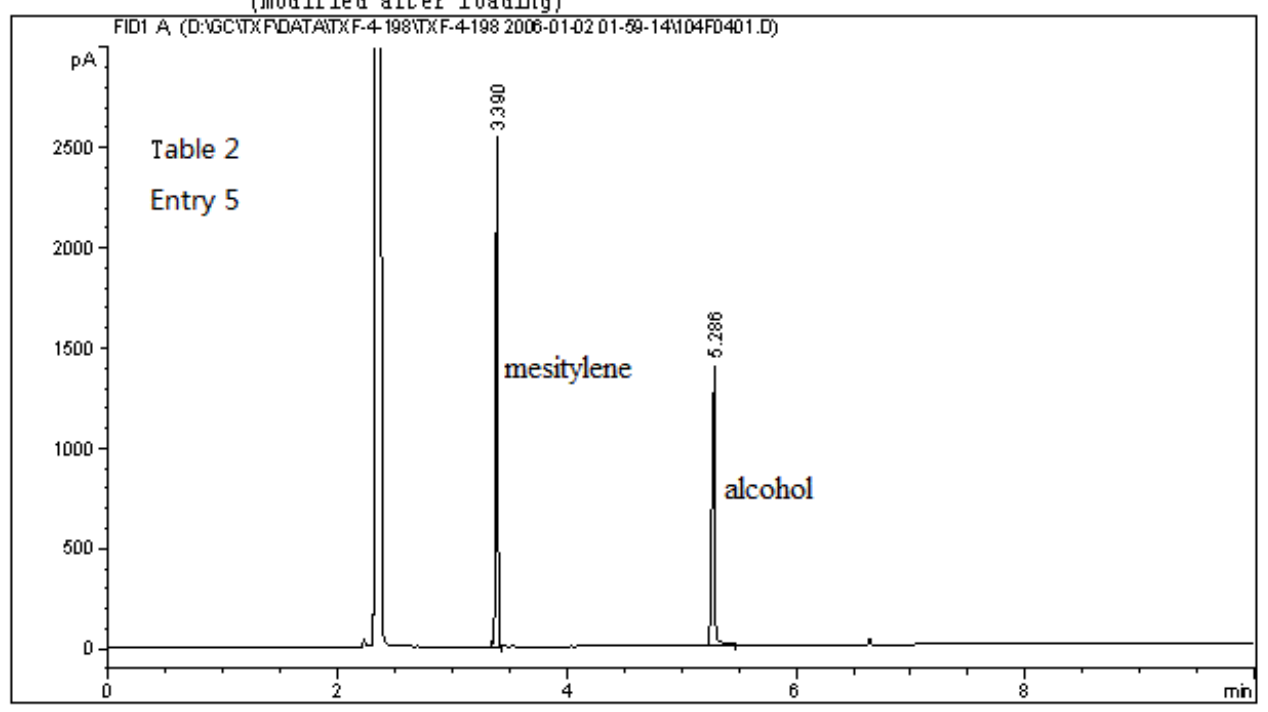

Area Percent Repor

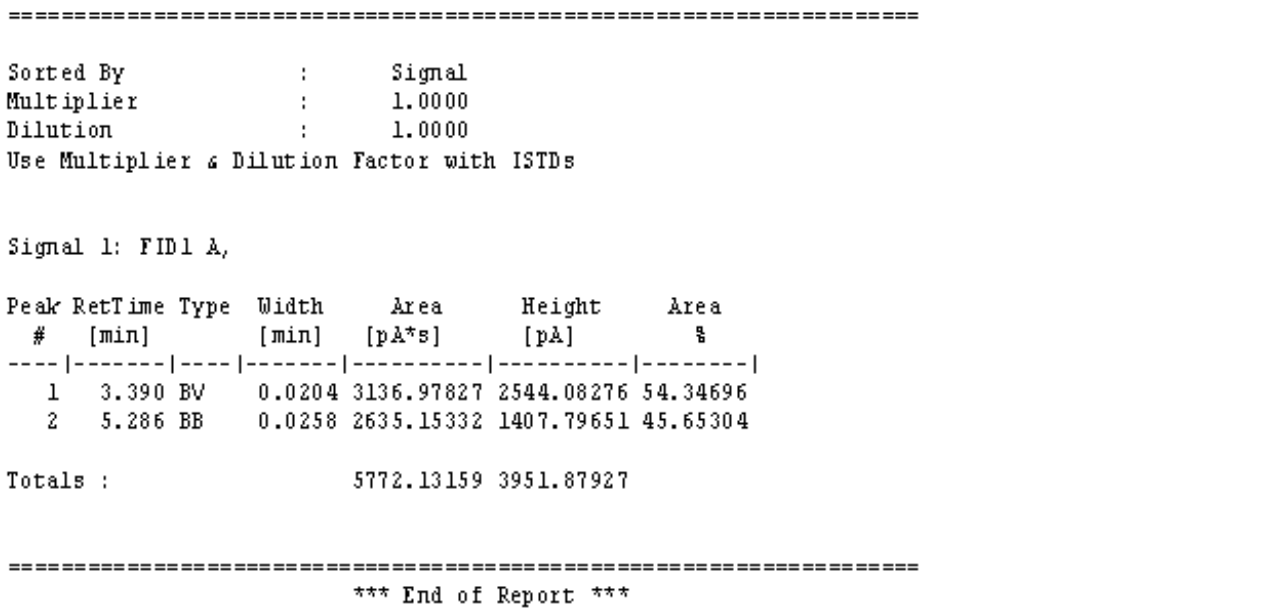




\section{<Sample Information>}

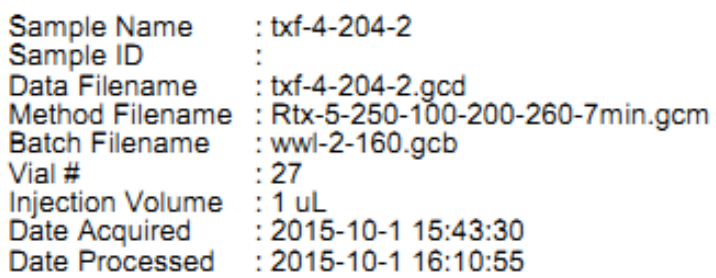

Sample Type : Unknown

Acquired by : : System Administrator Processed by : :System Administrator

\section{$<$ Chromatogram $>$}

$\mathrm{mV}$

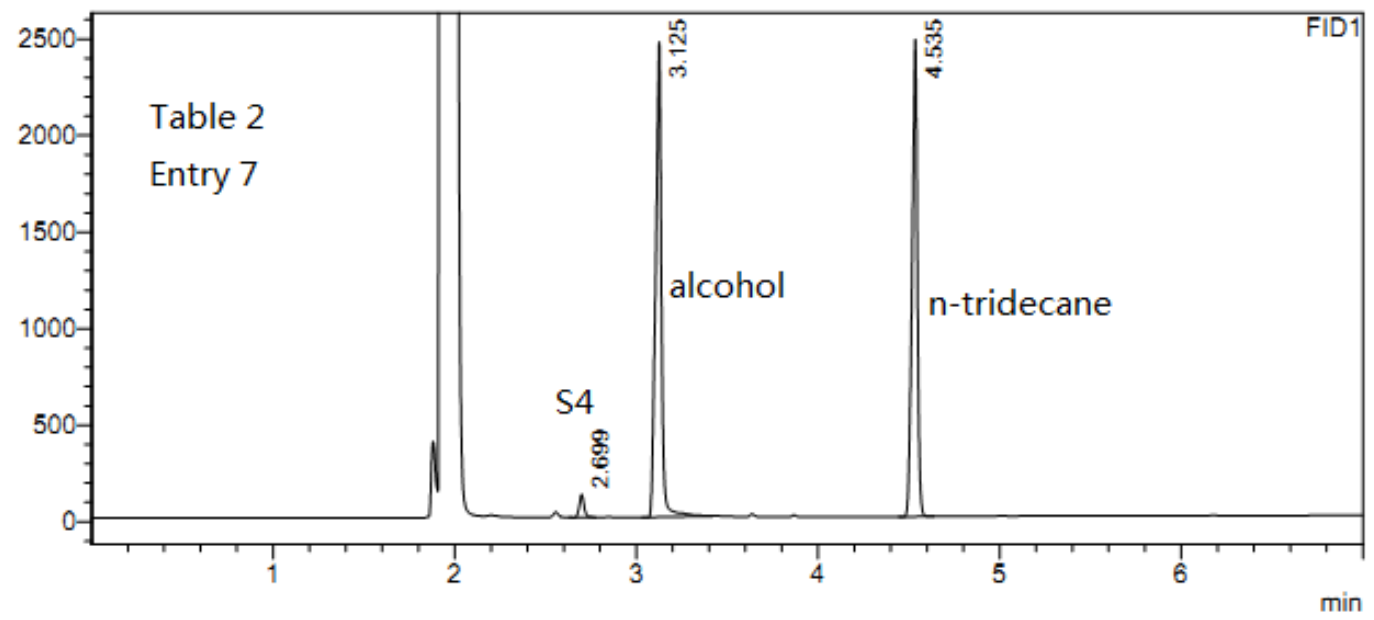

<Peak Table>

FID1

\begin{tabular}{|r|r|r|r|r|r|r|r|}
\hline Peak\# & Ret. Time & \multicolumn{1}{c|}{ Area } & \multicolumn{1}{c|}{ Height } & \multicolumn{1}{c|}{ Conc. } & Unit & Mark & Name \\
\hline 1 & 2.699 & 215394 & 117226 & 2.033 & & $\mathrm{M}$ & \\
\hline 2 & 3.125 & 5438854 & 2438704 & 51.327 & & $\mathrm{M}$ & \\
\hline 3 & 4.535 & 4942323 & 2442554 & 46.641 & & $\mathrm{M}$ & \\
\hline Total & & 10596571 & 4998484 & & & & \\
\hline
\end{tabular}


<Sample Information>

Sample Name : txf-5-2-5

Sample ID

Data Filename : txf-5-2-5.gcd

Method Filename : Rtx-5-250-100-200-260-9min.gcm

Batch Filename : txf-5-2.gcb

Vial \# : : 36

Injection Volume : $1 \mathrm{uL}$

Date Acquired : :2015-10-11 15:09:29

Date Processed : 2015-10-11 15:32:23

Sample Type : Unknown

Acquired by : : System Administrator

Processed by : System Administrator

\section{$<$ Chromatogram $>$}

$\mathrm{mV}$

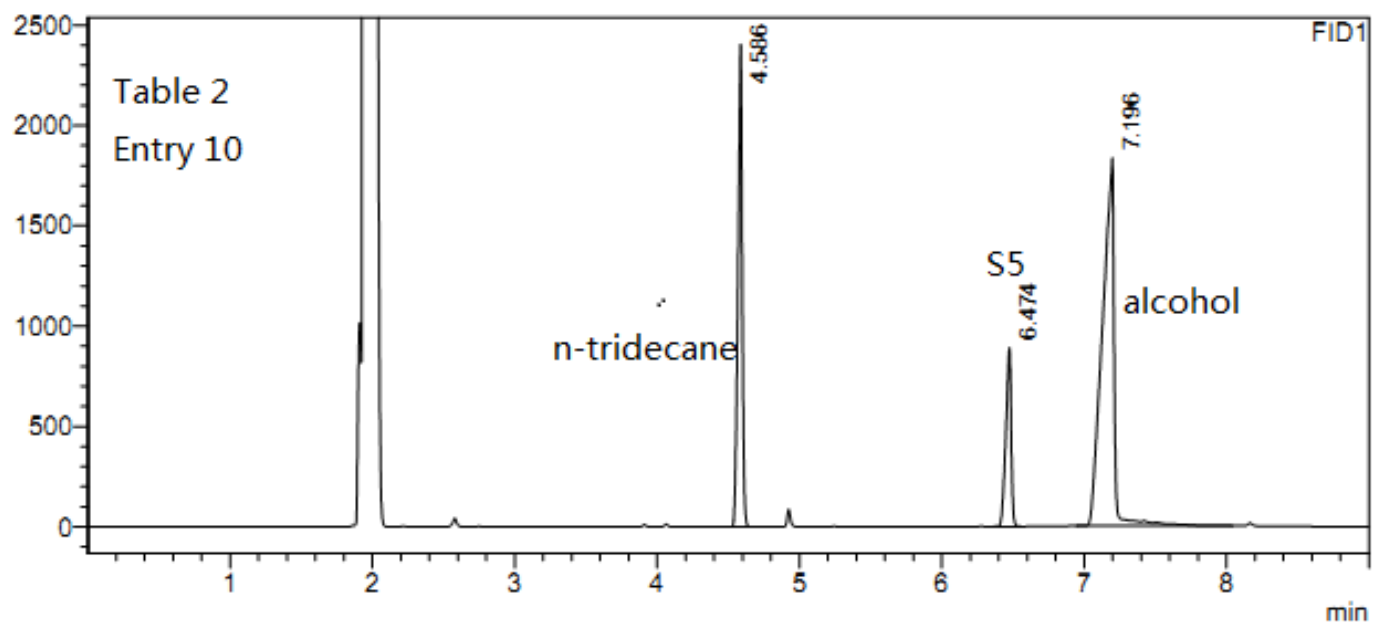

<Peak Table>

FID1

\begin{tabular}{|r|r|r|r|r|r|r|r|}
\hline Peak\# & Ret. Time & \multicolumn{1}{|c|}{ Area } & \multicolumn{1}{c|}{ Height } & \multicolumn{1}{c|}{ Conc. } & Unit & Mark & Name \\
\hline 1 & 4.586 & 4978592 & 2383736 & 27.900 & & $\mathrm{M}$ & \\
\hline 2 & 6.474 & 2320202 & 880418 & 13.003 & & $\mathrm{M}$ & \\
\hline 3 & 7.196 & 10545357 & 1812212 & 59.097 & & $\mathrm{M}$ & \\
\hline Total & & 17844150 & 5076366 & & & & \\
\hline
\end{tabular}


<Sample Information>

Sample Name : txf-4-189-1

Sample ID

Data Filename

: txf-4-189-1.gcd

Method Filename : Rtx-5-250-80(4min)-200-260-10min.gcm

Batch Filename : yc-3-97.gcb

Vial \# : 41

Injection Volume : 1 uL

Date Acquired : : 2015-9-15 19:46:10

Sample Type : Unknown

Date Processed : 2015-9-15 22:42:19

Acquired by : System Administrator

Processed by : : System Administrator

\section{$<$ Chromatogram $>$}

uV

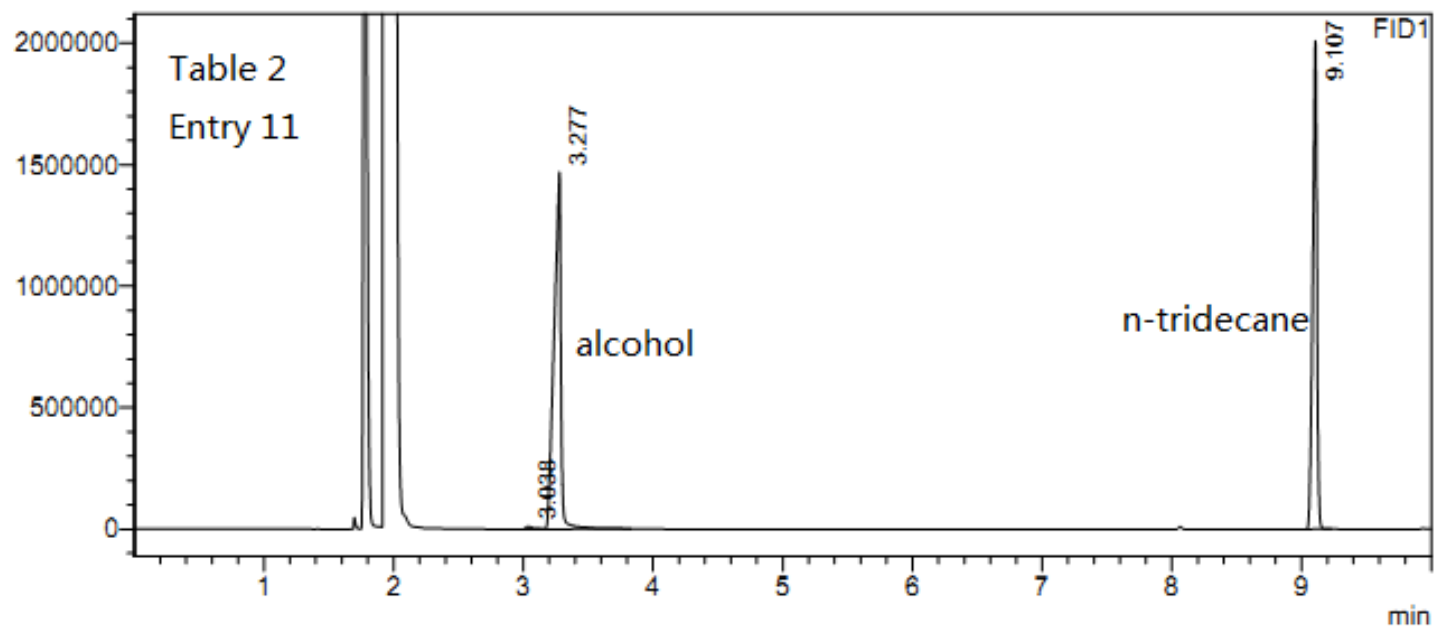

<Peak Table>

FID1

\begin{tabular}{|r|r|r|r|r|r|r|r|}
\hline Peak\# & Ret. Time & \multicolumn{1}{c|}{ Area } & Height & Conc. & Unit & Mark & Name \\
\hline 1 & 3.038 & 43039 & 10794 & 0.435 & & $\mathrm{M}$ & \\
\hline 2 & 3.277 & 5365908 & 1450336 & 54.288 & & $\mathrm{~V} \mathrm{M}$ & \\
\hline 3 & 9.107 & 4475162 & 1990039 & 45.276 & & $\mathrm{M}$ & \\
\hline Total & & 9884108 & 3451169 & & & & \\
\hline
\end{tabular}


<Sample Information>

Sample Name : : txf-5-3-7

Sample ID

Data Filename : txf-5-3-7.gcd

Method Filename : Rtx-5-250-100(3min)-200-260-8min.gcm

Batch Filename : txf-5-2.gcb

Vial \# : 32

Injection Volume : $1 \mathrm{uL}$

Date Acquired : :2015-10-11 12:23:47

Sample Type : Unknown

Date Processed : 2015-10-11 15:51:08

Acquired by : : System Administrator

Processed by : System Administrator

\section{$<$ Chromatogram $>$}

$\mathrm{mV}$

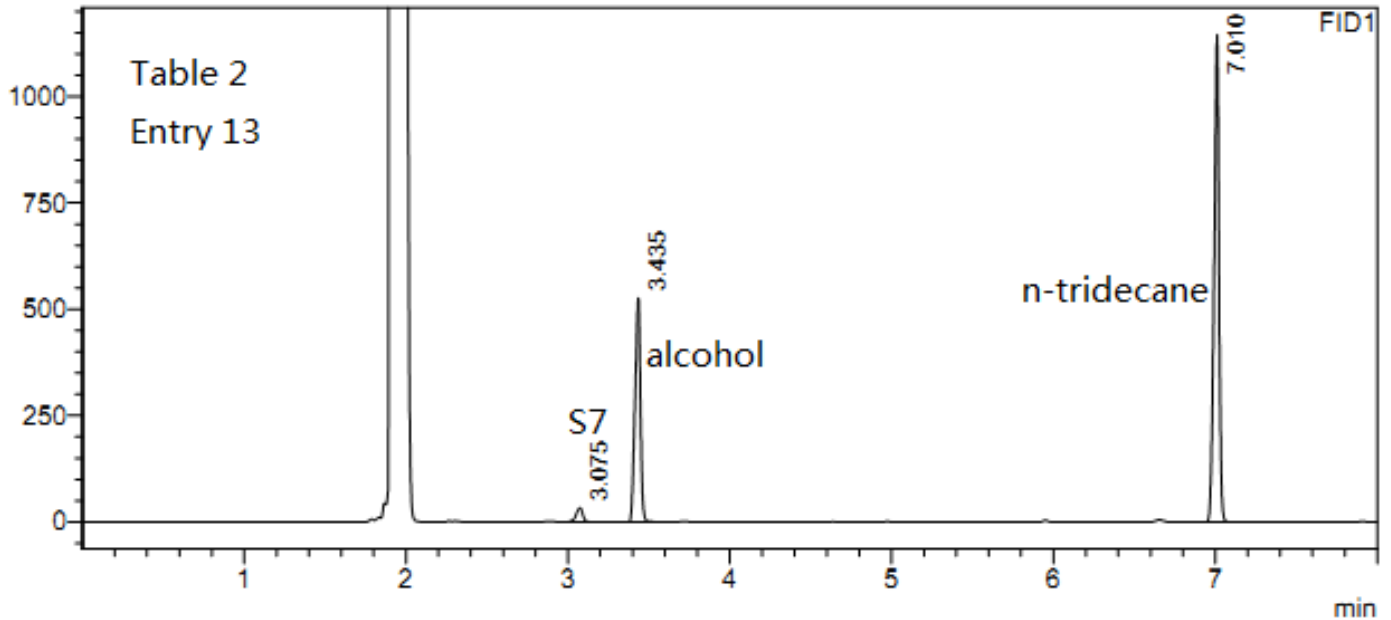

<Peak Table>

FID1

\begin{tabular}{|r|r|r|r|r|r|r|r|}
\hline Peak\# & Ret. Time & \multicolumn{1}{|c|}{ Area } & \multicolumn{1}{c|}{ Height } & \multicolumn{1}{c|}{ Conc. } & Unit & Mark & Name \\
\hline 1 & 3.075 & 94053 & 33136 & 2.537 & & $\mathrm{M}$ & \\
\hline 2 & 3.435 & 1213256 & 519012 & 32.732 & & $\mathrm{M}$ & \\
\hline 3 & 7.010 & 2399327 & 1112261 & 64.731 & & $\mathrm{M}$ & \\
\hline Total & & 3706636 & 1664409 & & & & \\
\hline
\end{tabular}




\section{<Sample Information>}

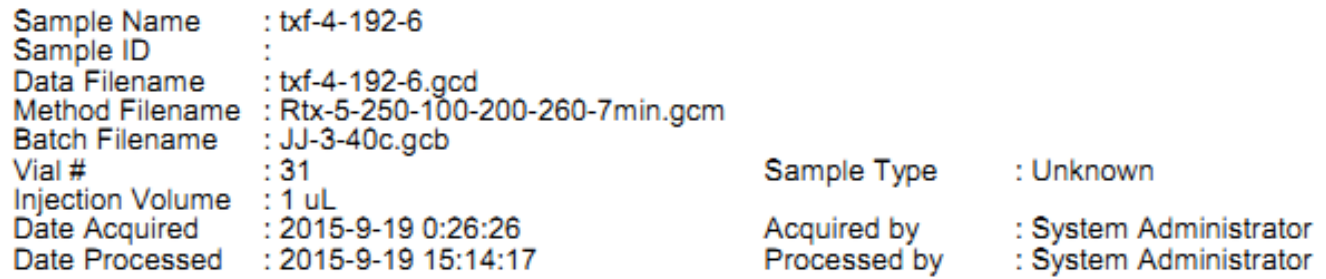

\section{$<$ Chromatogram>}

u

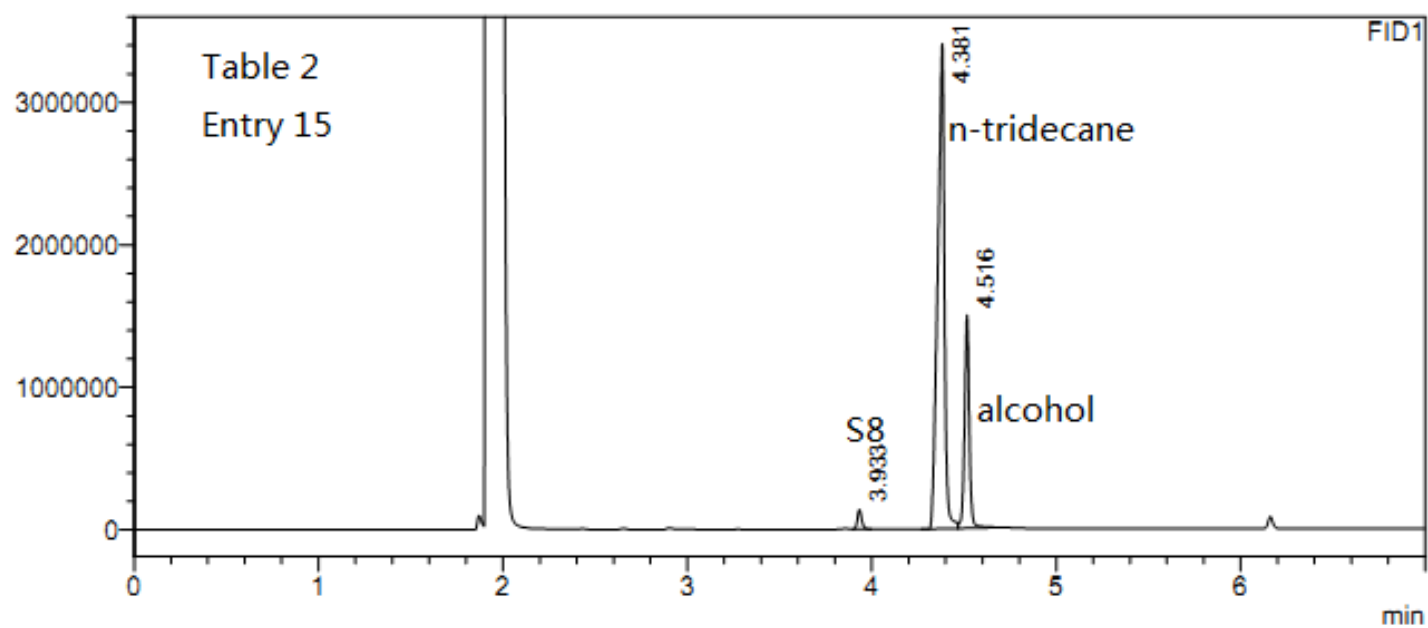

<Peak Table>

FID1

\begin{tabular}{|r|r|r|r|r|r|r|r|}
\hline Peak\# & Ret. Time & \multicolumn{1}{c|}{ Area } & Height & Conc. & Unit & Mark & Name \\
\hline 1 & 3.933 & 219386 & 136412 & 1.906 & & $\mathrm{M}$ & \\
\hline 2 & 4.381 & 8630052 & 3351114 & 74.958 & & $\mathrm{M}$ & \\
\hline 3 & 4.516 & 2663712 & 1474787 & 23.136 & & $\mathrm{~V} \mathrm{M}$ & \\
\hline Total & & 11513151 & 4962313 & & & & \\
\hline
\end{tabular}




\section{<Sample Information>}

Sample Name

Sample ID

txf-4-192-2

Data Filename : txf-4-192-2.gcd

Method Filename : Rtx-5-250-80(4min)-200-260-10min.gcm

Batch Filename : JJ-3-40c.gcb

Vial \# : 27

Injection Volume : $1 \mathrm{uL}$

Date Acquired : 2015-9-18 23:27:18

Date Processed : 2015-9-19 15:13:10

Sample Type : Unknown

Acquired by : : System Administrator

Processed by : : System Administrator

\section{$<$ Chromatogram $>$}

uV

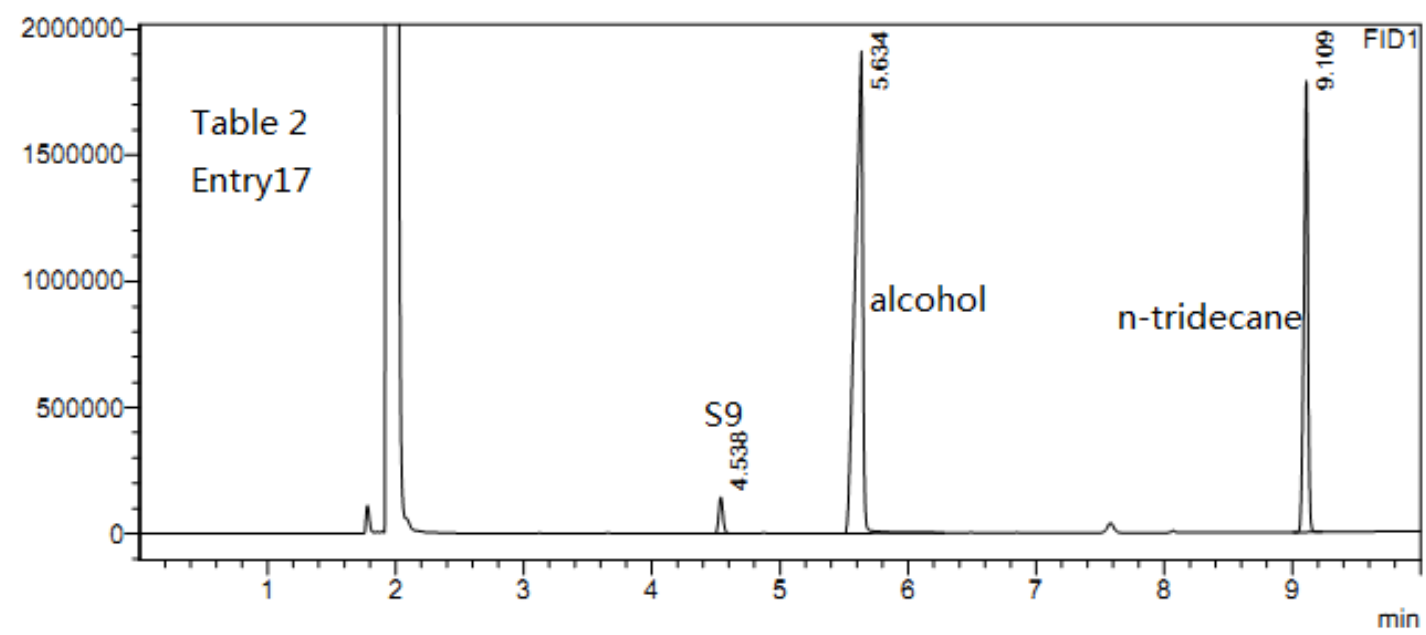

$\langle$ Peak Table>

FID1

\begin{tabular}{|r|r|r|r|r|r|r|r|}
\hline Peak\# & Ret. Time & \multicolumn{1}{c|}{ Area } & Height & Conc. & Unit & Mark & Name \\
\hline 1 & 4.538 & 325185 & 143384 & 2.694 & & $\mathrm{M}$ & \\
\hline 2 & 5.634 & 7940151 & 1901378 & 65.786 & & $\mathrm{M}$ & \\
\hline 3 & 9.109 & 3804272 & 1759590 & 31.519 & & $\mathrm{M}$ & \\
\hline Total & & 12069608 & 3804352 & & & &
\end{tabular}


<Sample Information>

Sample Name

Sample ID

Data Filename

: txf-4-204-5

: txf-4-204-5.gcd

Method Filename : Rtx-5-250-130-260-7min.gcm

Batch Filename : wwl-2-160.gcb

Vial \# : 30

Injection Volume : $1 \mathrm{uL}$

Date Acquired : :2015-10-1 16:28:15

Date Processed : : 2016-1-16 15:09:11

Sample Type : Unknown

Acquired by : System Administrator

Processed by : System Administrator

\section{$<$ Chromatogram $>$}

$\mathrm{mV}$

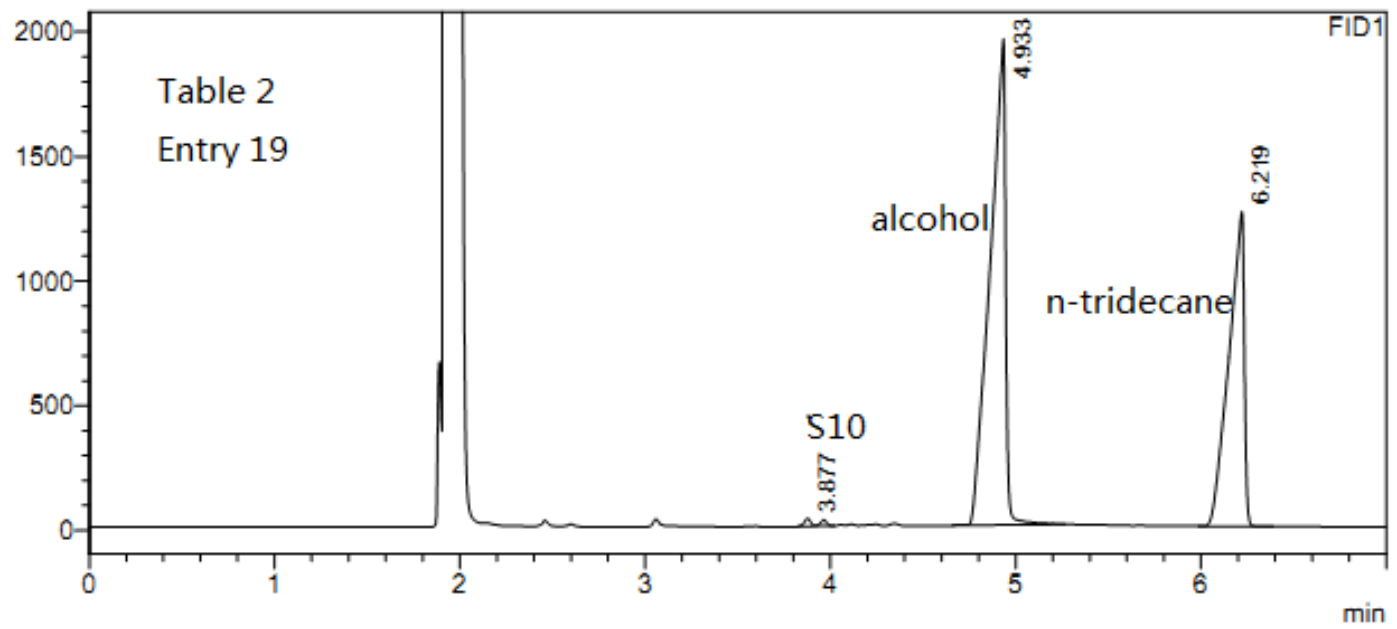

<Peak Table>

FID1

\begin{tabular}{|r|r|r|r|r|r|r|r|}
\hline Peak\# & Ret. Time & \multicolumn{1}{c|}{ Area } & Height & Conc. & Unit & Mark & Name \\
\hline 1 & 3.877 & 133671 & 31654 & 0.716 & & $\mathrm{M}$ & \\
\hline 2 & 4.933 & 11278784 & 1925160 & 60.447 & & $\mathrm{M}$ & \\
\hline 3 & 6.219 & 7246383 & 1256880 & 38.836 & & $\mathrm{M}$ & \\
\hline Total & & 18658837 & 3213695 & & & & \\
\hline
\end{tabular}


Data File E: \DATA \TXYUGZ-1\QD.D

Sample Name: 0 (
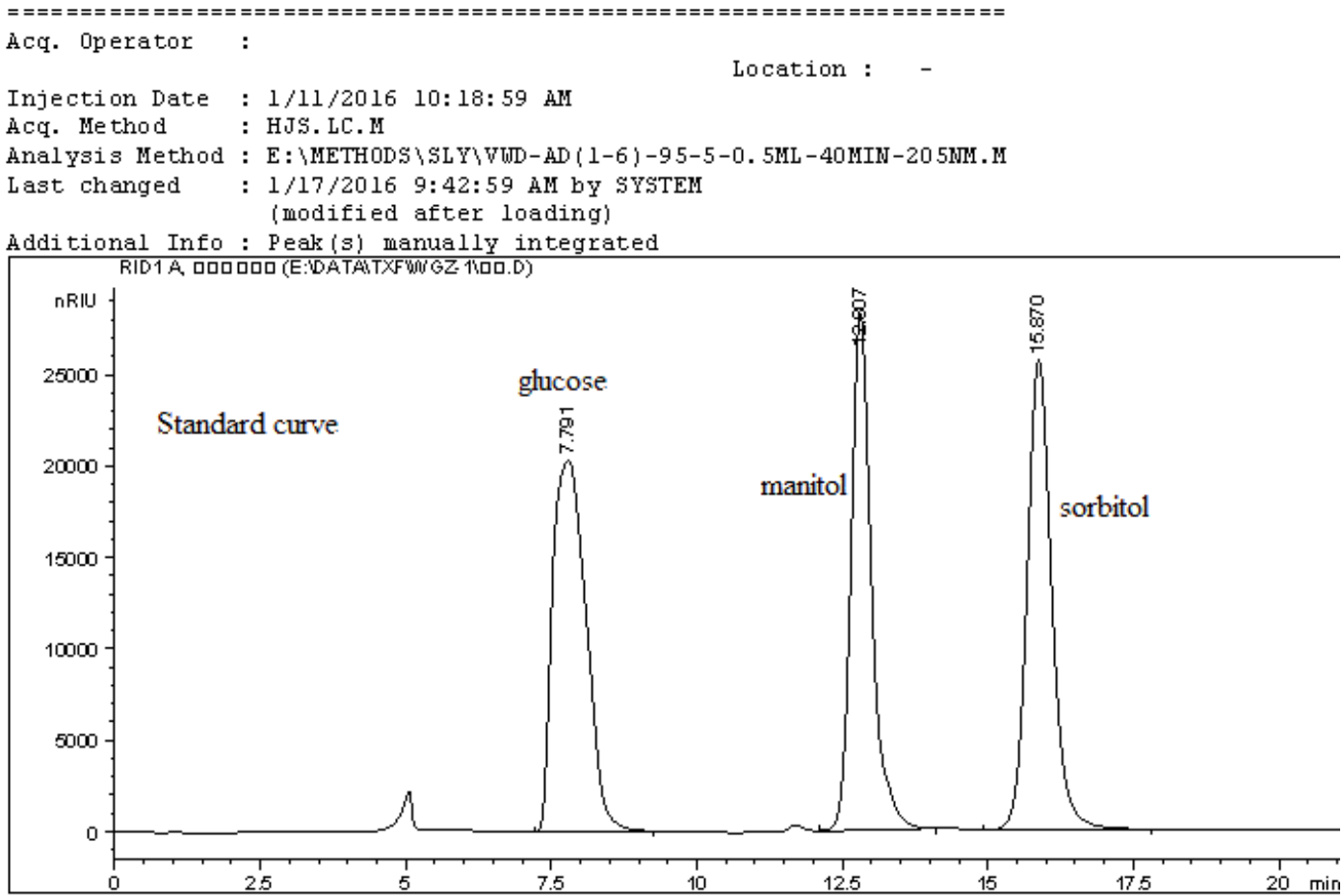

Area Percent Report

\begin{tabular}{|c|c|c|}
\hline sorted By & : & Signal \\
\hline Multiplier & : & 1.0000 \\
\hline Dilution & : & 1.0000 \\
\hline
\end{tabular}

Do not use Multiplier \& Dilution Factor with ISTDs

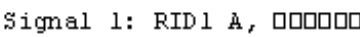

\begin{tabular}{|c|c|c|c|c|c|c|}
\hline $\begin{array}{c}\text { Peak } \\
\#\end{array}$ & $\begin{array}{l}\text { RetTime } \\
\text { [min] }\end{array}$ & Type & $\begin{array}{l}\text { Width } \\
\text { [min] }\end{array}$ & $\begin{array}{c}\text { Area } \\
\text { [nRIU*s] }\end{array}$ & $\begin{array}{l}\text { Height } \\
\text { [nRIU] }\end{array}$ & $\begin{array}{c}\text { Area } \\
\frac{8}{8}\end{array}$ \\
\hline 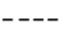 & - & -1 & 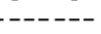 & --- & -- & ----1 \\
\hline 1 & 7.791 & $\mathrm{MM} \mathrm{R}$ & 0.6662 & 8.13230 e 5 & $2.03437 \mathrm{e} 4$ & 35.9657 \\
\hline 2 & 12.807 & $\mathrm{MM} R$ & 0.4085 & 6.92007 e 5 & $2.82313 \mathrm{e} 4$ & 30.6045 \\
\hline 3 & 15.870 & $\mathrm{MM} R$ & 0.4905 & 7.55889 e 5 & $2.56864 \mathrm{e} 4$ & 33.4298 \\
\hline
\end{tabular}

Totals : $\quad 2.26113 \mathrm{e} 6 \quad 7.42615 \mathrm{e} 4$

ネネー End of Report *ネ 
Data File E: \DATA $\backslash$ TXF $\backslash$ UGZ-1\l.D

Sample Name: $1 \#$
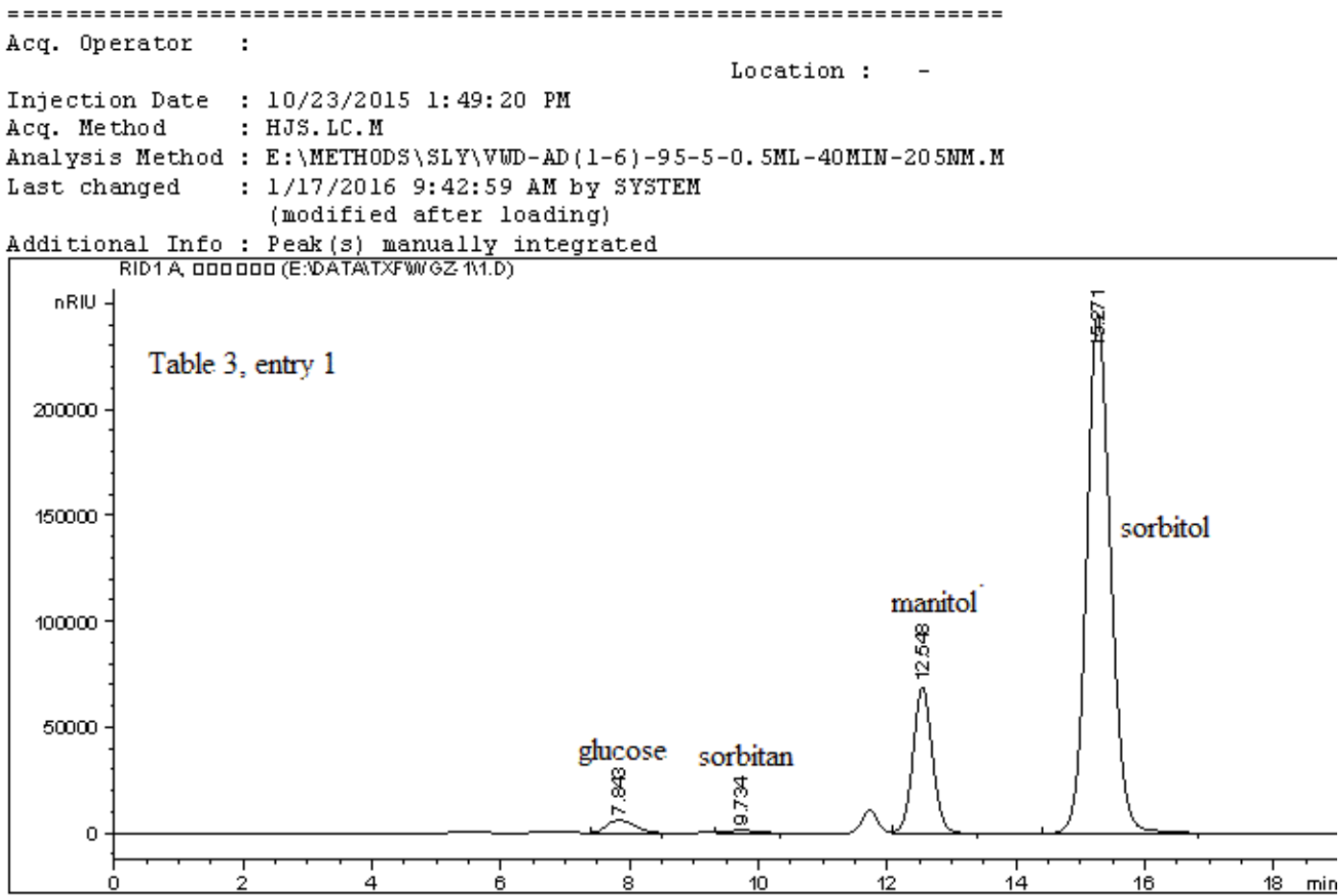

Area Percent Report

\begin{tabular}{|c|c|c|}
\hline Sorted By & signal & \\
\hline Multiplier & 1. 0000 & \\
\hline Dilution & 1.0000 & \\
\hline Sample Amount: & $: \quad 20.00000$ & (not used in calc.) \\
\hline
\end{tabular}

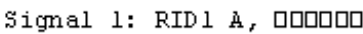

\begin{tabular}{|c|c|c|c|c|c|c|}
\hline $\begin{array}{c}\text { Peak } \\
\#\end{array}$ & $\begin{array}{c}\text { Re tTime } \\
\text { [ min] }\end{array}$ & Type & $\begin{array}{l}\text { Width } \\
\text { [min] }\end{array}$ & $\begin{array}{c}\text { Area } \\
{\left[\mathrm{nRIU}^{*} \mathrm{~s}\right]}\end{array}$ & $\begin{array}{l}\text { Height } \\
\text { [nRIU] }\end{array}$ & $\begin{array}{c}\text { Area } \\
\stackrel{4}{*}\end{array}$ \\
\hline & - & & 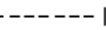 & -- & ------ & 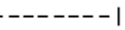 \\
\hline 1 & 7.843 & $\mathrm{MM} R$ & 0.5006 & $1.77336 \mathrm{e} 5$ & 5904.19238 & 2.2000 \\
\hline 2 & 9.734 & $\mathrm{MM} R$ & 0.5331 & 3. $63695 \mathrm{e} 4$ & 1137.00305 & 0.4512 \\
\hline 3 & 12.548 & FM R & 0.3545 & 1. $47159 \mathrm{e} 6$ & $6.91824 \mathrm{e} 4$ & 18.2561 \\
\hline 4 & 15.271 & $\mathrm{MM} R$ & 0.4336 & $6.37550 \mathrm{e} 6$ & $2.45056 \mathrm{e} 5$ & 79.0927 \\
\hline & . & & & 06080 e 6 & $280 \mathrm{e} 5$ & \\
\hline
\end{tabular}

ネネネ End of Report ネネ 
Data File E: \DATA $\backslash T X F \backslash W G Z-1 \backslash 2 . D$

Sample Name: $4 \#$
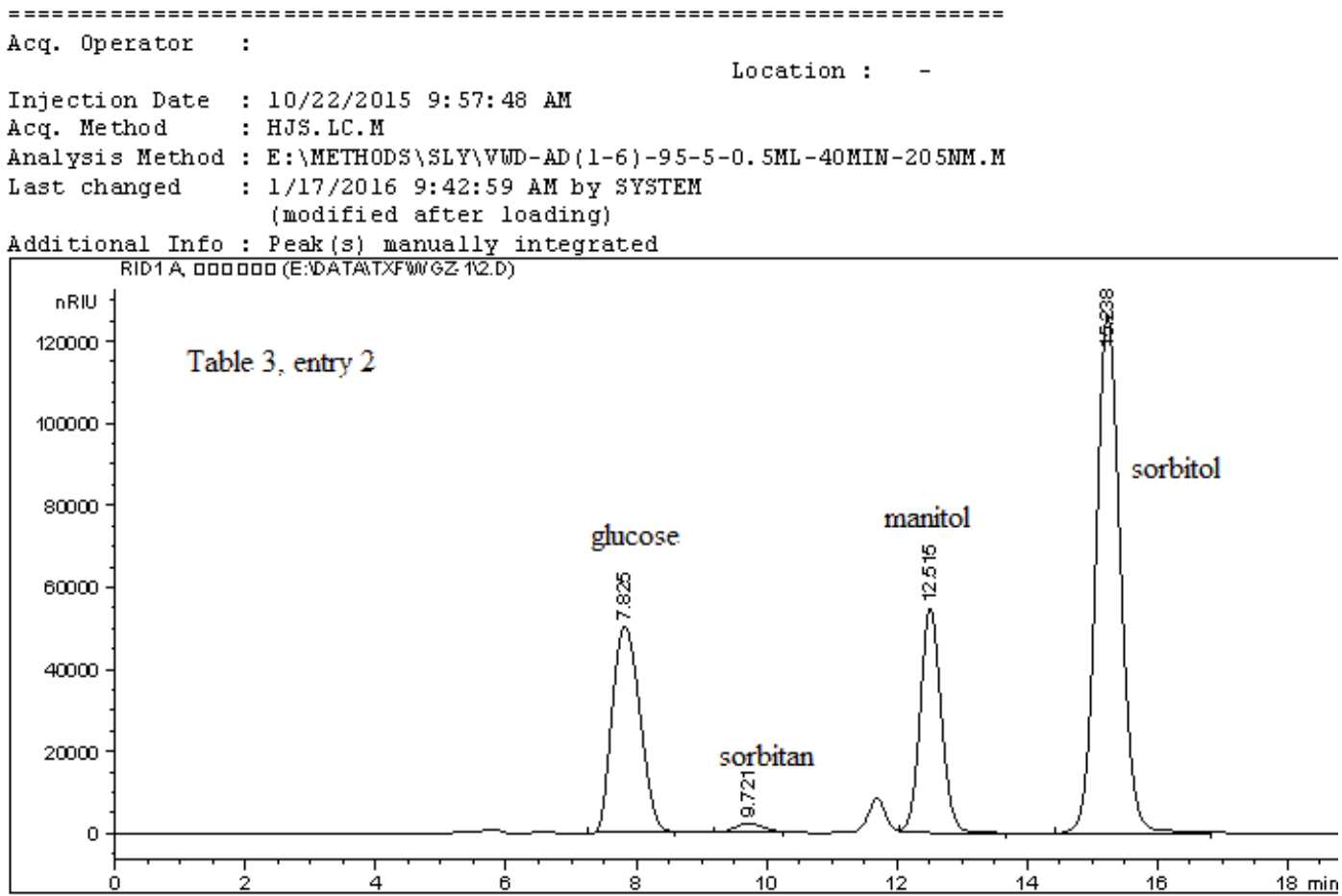

Area Percent Report

\begin{tabular}{|c|c|c|}
\hline Sorted By & signal & \\
\hline Multiplier & 1. 0000 & \\
\hline Dilution & 1.0000 & \\
\hline Sample Amount: & $: \quad 20.00000$ & (not used in calc.) \\
\hline
\end{tabular}

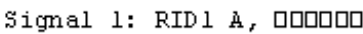

\begin{tabular}{|c|c|c|c|c|c|c|}
\hline $\begin{array}{c}\text { Peak } \\
\#\end{array}$ & $\begin{array}{c}\text { Re tTime } \\
\text { [ min] }\end{array}$ & Type & $\begin{array}{l}\text { Width } \\
\text { [min] }\end{array}$ & $\begin{array}{c}\text { Area } \\
{\left[\mathrm{nRIU}^{*} \mathrm{~s}\right]}\end{array}$ & $\begin{array}{l}\text { Height } \\
\text { [nRIU] }\end{array}$ & $\begin{array}{c}\text { Area } \\
\stackrel{4}{*}\end{array}$ \\
\hline & 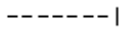 & & & -- & ------ & ----- \\
\hline 1 & 7.825 & $\mathrm{MM} R$ & 0.4916 & 1. $48126 \mathrm{e} 6$ & $5.02189 \mathrm{e} 4$ & 24.4097 \\
\hline 2 & 9.721 & $\mathrm{MM} R$ & 0.4880 & $6.38078 \mathrm{e} 4$ & 2179.20923 & 1.0515 \\
\hline 3 & 12.515 & FM R & 0.3763 & $1.23455 \mathrm{e} 6$ & $5.46753 \mathrm{e} 4$ & 20.3442 \\
\hline 4 & 15.238 & $\mathrm{MM} R$ & 0.4339 & $3.28870 \mathrm{e} 6$ & $1.26319 \mathrm{e} 5$ & 54.1945 \\
\hline & 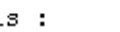 & & & 32 e 6 & $392 \mathrm{e} 5$ & \\
\hline
\end{tabular}

ネネネ End of Report ネネ 
Data File E: \DATA $\backslash T X F \backslash W G Z-1 \backslash 3 . D$

Sample Name: 10\#
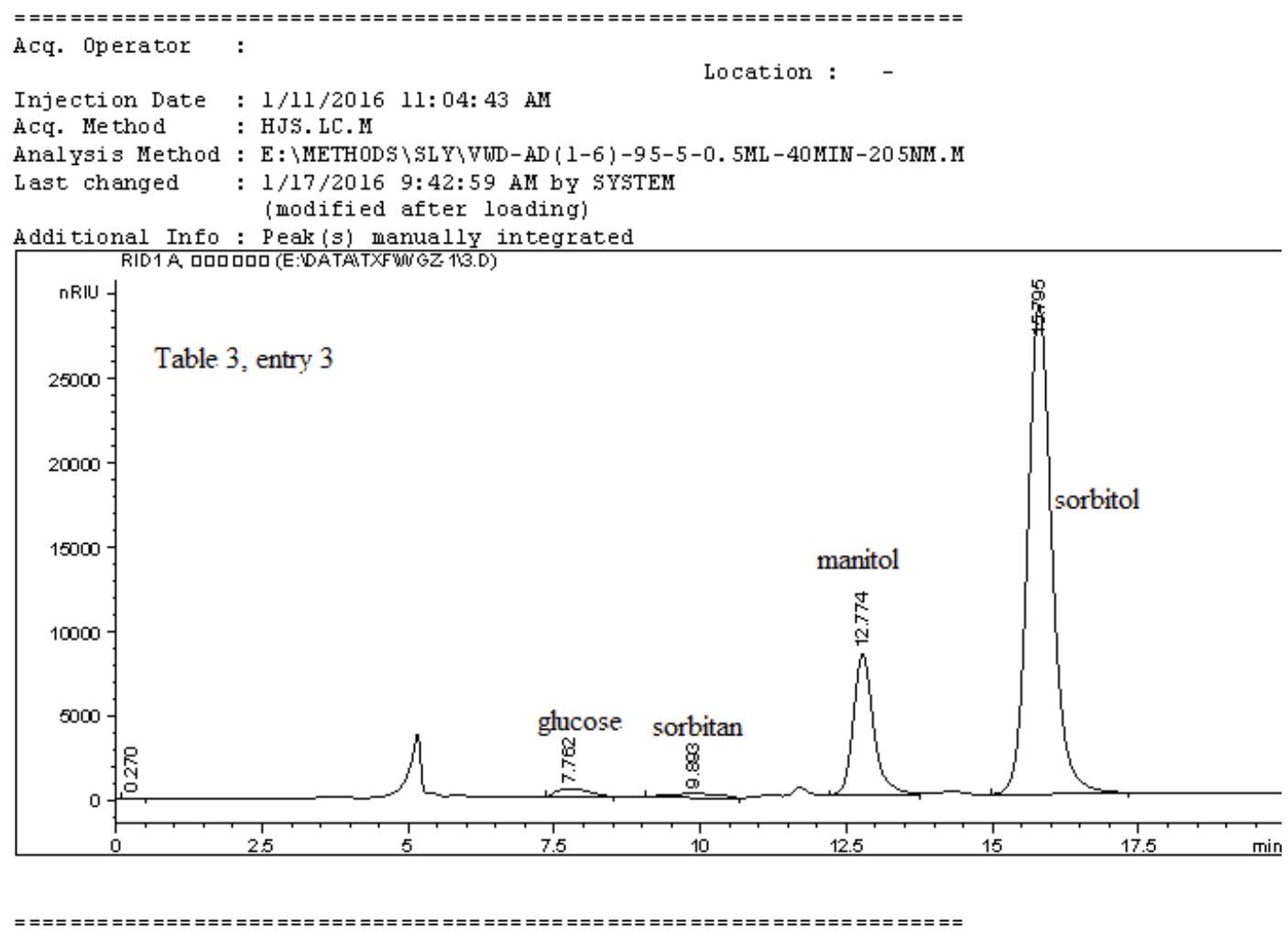

Area Percent Report

\begin{tabular}{|c|c|c|}
\hline sorted By & : & Signal \\
\hline Multiplier & : & 1.0000 \\
\hline Dilution & : & 1.0000 \\
\hline
\end{tabular}

Do not use Multiplier \& Dilution Factor with ISTDs

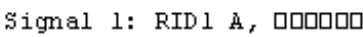

\begin{tabular}{|c|c|c|c|c|c|c|}
\hline $\begin{array}{c}\text { Peak } \\
\#\end{array}$ & $\begin{array}{l}\text { RetTime } \\
\text { [min] }\end{array}$ & Type & $\begin{array}{l}\text { Width } \\
\text { [min] }\end{array}$ & $\begin{array}{c}\text { Area } \\
\text { [nRIU*s] }\end{array}$ & $\begin{array}{l}\text { Height } \\
\text { [nRIU] }\end{array}$ & $\begin{array}{c}\text { Àrea } \\
\stackrel{8}{*}\end{array}$ \\
\hline & & & & & 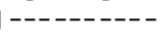 & \\
\hline 1 & 0.270 & BB & 0.1847 & 86.13216 & 5.85142 & $8.033 \mathrm{e}-3$ \\
\hline 2 & 7.762 & $\mathrm{MM} R$ & 0.6 & 1.746 & 457.6 & 85 \\
\hline 3 & 9.893 & $\mathrm{MM} R$ & 0.9419 & 1. $50360 \mathrm{e} 4$ & 266.04541 & 1. 4023 \\
\hline 4 & 12.774 & $\mathrm{MM} \mathrm{R}$ & 0.4011 & $2.00976 \mathrm{e} 5$ & 8351.91016 & 18.7441 \\
\hline 5 & 15.795 & $\mathrm{MM} \mathrm{R}$ & 0.4811 & 8.38648 e 5 & $2.9053 \mathrm{le} 4$ & 78.2170 \\
\hline \multicolumn{7}{|c|}{ tals } \\
\hline
\end{tabular}

ネネ* End of Report *ネ 
Data File E: \DATA \TXF\UGZ-1 $\backslash 4 . D$

Sample Name: 12\#
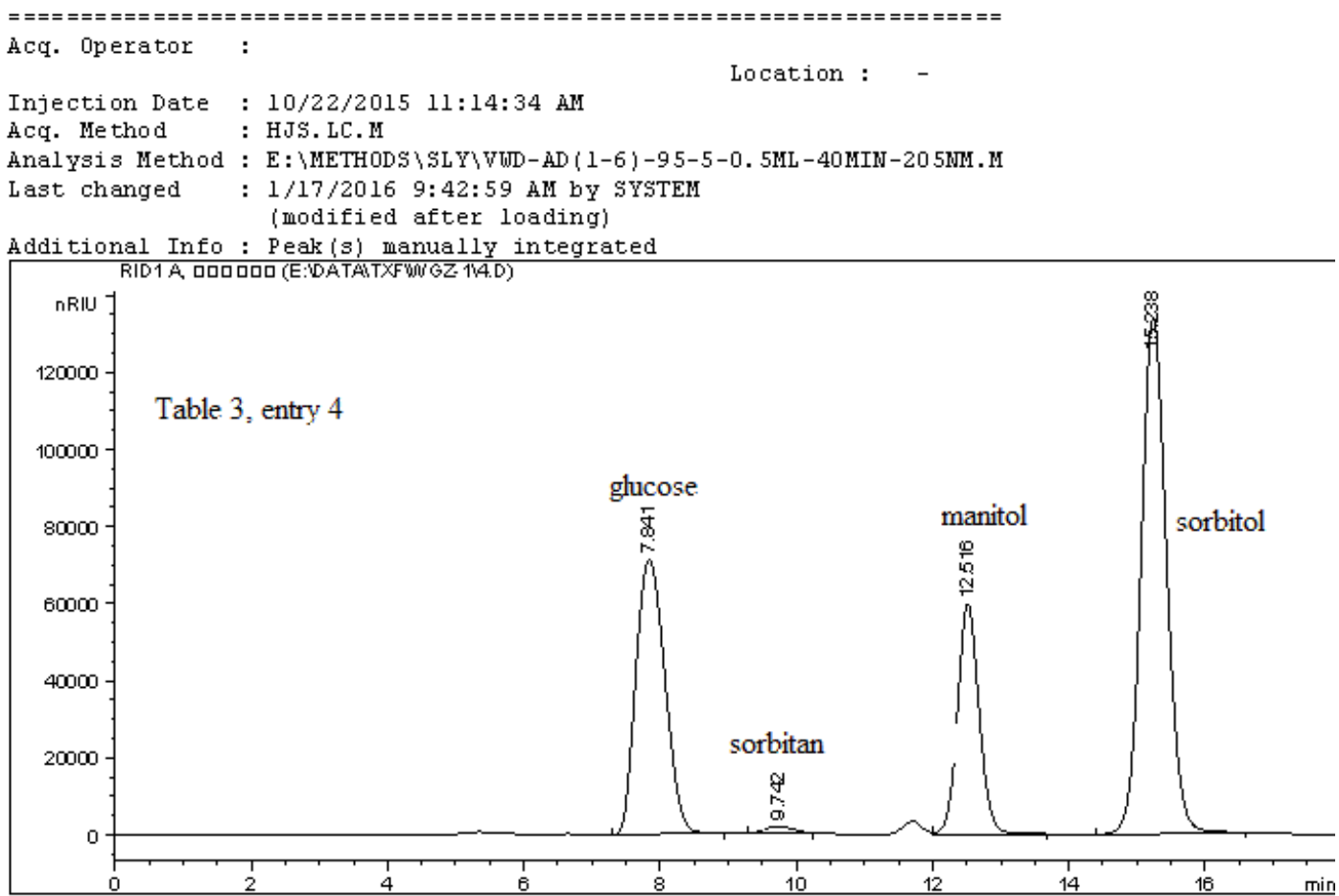

Area Percent Report

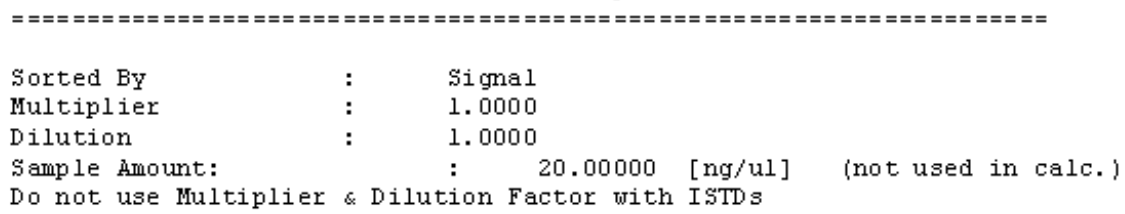

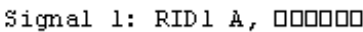

\begin{tabular}{|c|c|c|c|c|c|c|}
\hline $\begin{array}{c}\text { Peak } \\
\#\end{array}$ & $\begin{array}{c}\text { Re tTime } \\
\text { [ min] }\end{array}$ & Type & $\begin{array}{l}\text { Width } \\
\text { [min] }\end{array}$ & $\begin{array}{c}\text { Area } \\
{\left[\mathrm{nRIU}^{*} \mathrm{~s}\right]}\end{array}$ & $\begin{array}{l}\text { Height } \\
\text { [nRIU] }\end{array}$ & $\begin{array}{c}\text { Area } \\
\stackrel{4}{*}\end{array}$ \\
\hline & . & & & ------- & ------- & 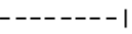 \\
\hline 1 & 7.841 & $\mathrm{MM} R$ & 0.4922 & $2.10277 \mathrm{e} 6$ & $7.12064 \mathrm{e} 4$ & 30.2913 \\
\hline 2 & 9.742 & $\mathrm{MM} R$ & 0.4746 & $5.38023 e^{4}$ & 1889.52588 & 0.7750 \\
\hline 3 & 12.516 & FM R & 0.3672 & $1.31541 \mathrm{e} 6$ & $5.97044 \mathrm{e} 4$ & 18.9490 \\
\hline 4 & 15.238 & $\mathrm{MM} R$ & 0.4317 & 3. $46986 \mathrm{e} 6$ & $1.33968 \mathrm{e} 5$ & 49.9847 \\
\hline & 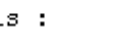 & & & 4 e 6 & 69 es & \\
\hline
\end{tabular}

ネネネ End of Report ネネ 
Data File E: \DATA \TXF\UGZ-1\5.D

Sample Name: $18 \#$
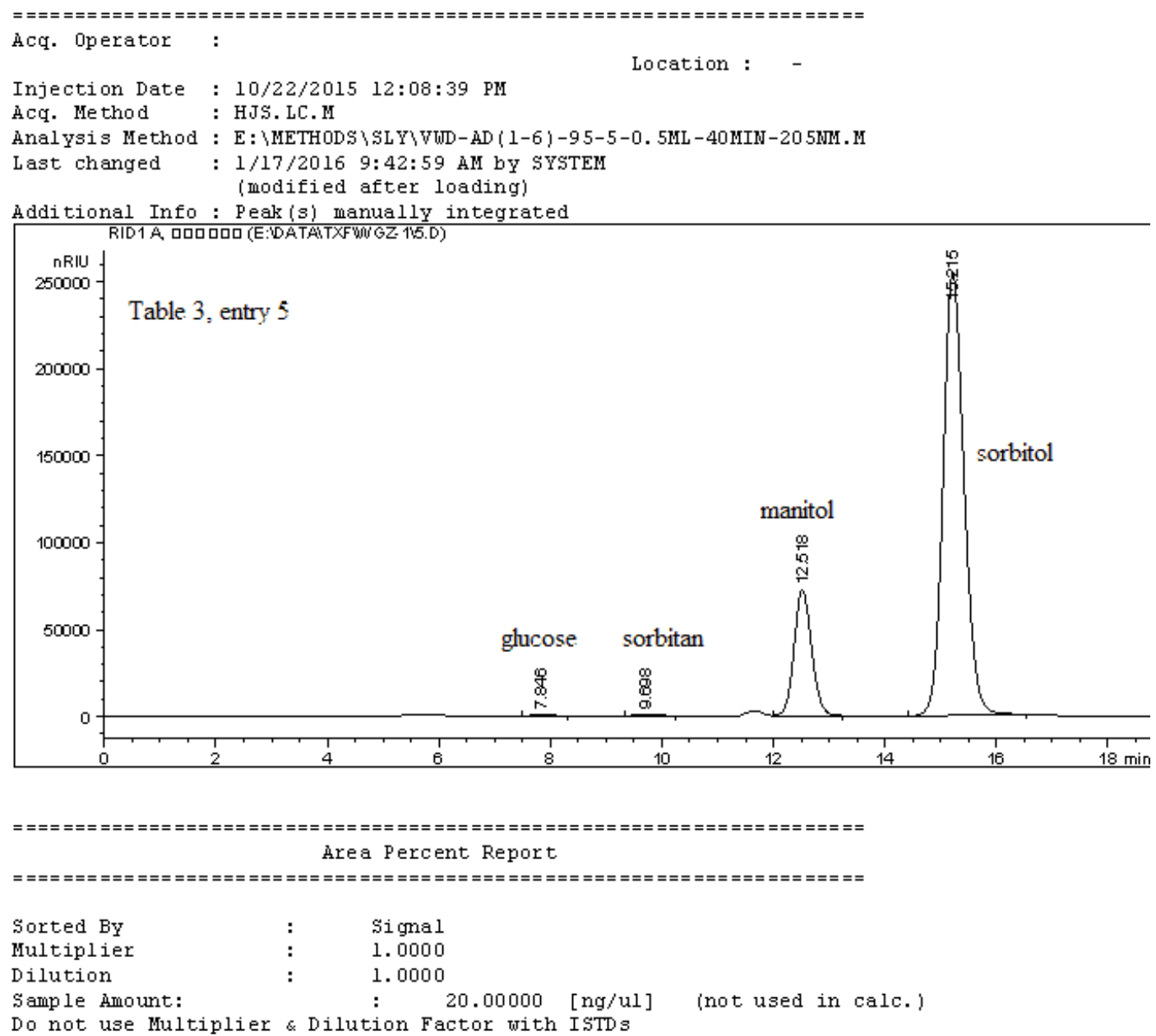

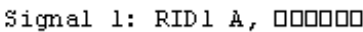

\begin{tabular}{|c|c|c|c|c|c|c|}
\hline $\begin{array}{c}\text { Peak } \\
\#\end{array}$ & $\begin{array}{c}\text { Re tTime } \\
\text { [min] }\end{array}$ & Type & $\begin{array}{l}\text { Width } \\
\text { [min] }\end{array}$ & $\begin{array}{c}\text { Area } \\
{\left[\mathrm{nRIU}^{*} \mathrm{~s}\right]}\end{array}$ & $\begin{array}{l}\text { Height } \\
\text { [nRIU] }\end{array}$ & $\begin{array}{c}\text { Area } \\
\stackrel{4}{\$}\end{array}$ \\
\hline 1 & 7.846 & $\mathrm{MM} \mathrm{R}$ & 0.4792 & 2. $44137 e 4$ & 849.18799 & 0.2956 \\
\hline 2 & 9.698 & $\mathrm{MM} R$ & 0.5311 & 2. $88955 \mathrm{e} 4$ & 906.72150 & 0.3499 \\
\hline 3 & 12.518 & $\mathrm{MM} \mathrm{R}$ & 0.3636 & 1. $58067 \mathrm{e} 6$ & $7.24609 \mathrm{e} 4$ & 19.1392 \\
\hline 4 & 15.215 & $M M R$ & 0.4342 & $6.62482 \mathrm{e} 6$ & 2.5430 le 5 & 80.2153 \\
\hline & . & & & 25880 e 6 & $.28517 \mathrm{e} 5$ & \\
\hline
\end{tabular}

\section{\#ネ Fnd of Report \#ネ}


Data File E: \DATA $\backslash T X F \backslash W G Z-1 \backslash 6 . D$

Sample Name: $1 \#$
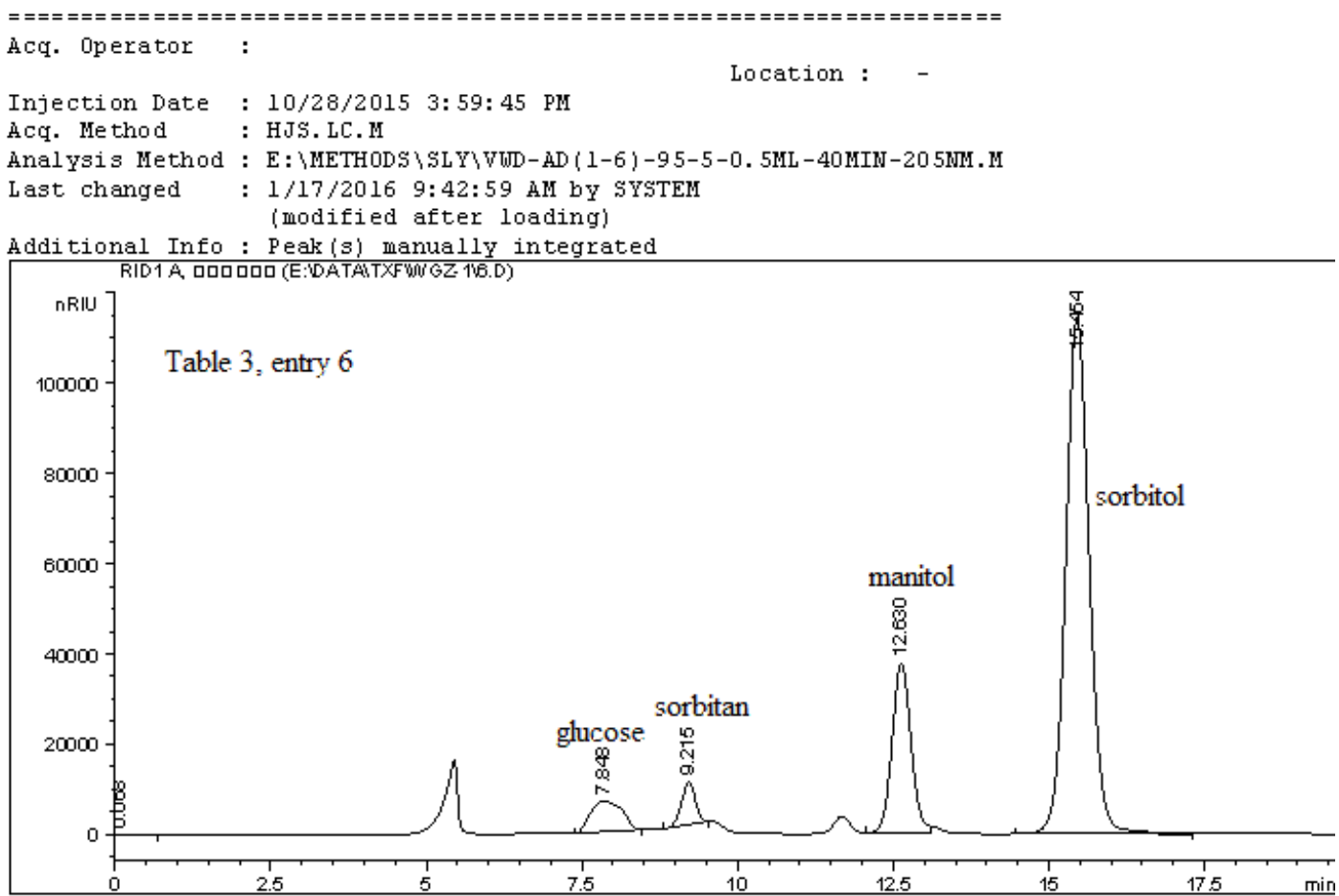

Area Percent Report

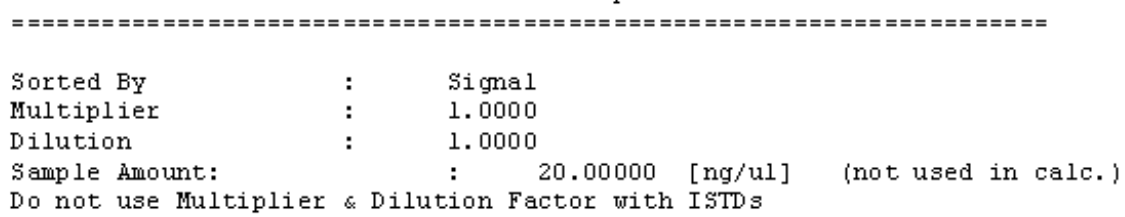

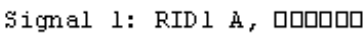

\begin{tabular}{|c|c|c|c|c|c|c|}
\hline $\begin{array}{c}\text { Peak } \\
\#\end{array}$ & $\begin{array}{c}\text { Re tTime } \\
\text { [min] }\end{array}$ & Type & $\begin{array}{l}\text { Width } \\
\text { [min] }\end{array}$ & $\begin{array}{c}\text { Area } \\
{\left[\mathrm{nRIU}{ }^{*} \mathrm{~s}\right]}\end{array}$ & $\begin{array}{l}\text { Height } \\
\text { [nRIU] }\end{array}$ & $\begin{array}{c}\text { Arrea } \\
\stackrel{4}{*}\end{array}$ \\
\hline & 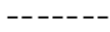 & & --- & |-------- & ------- & ----- \\
\hline 1 & 0.068 & $\mathrm{BB}$ & 0.6106 & 137.25935 & 2.64131 & $3.296 \mathrm{e}-3$ \\
\hline 2 & 7.848 & $\mathrm{MMR}$ & 0.5733 & 2.36862 e 5 & 6886.02051 & 5.6885 \\
\hline 3 & 9.215 & $\mathrm{MM} R$ & 0.2561 & 1. $46812 \mathrm{e} 5$ & 9554.56250 & 3.5258 \\
\hline 4 & 12.630 & $M F R$ & 0.3520 & 7. 9609 le 5 & $3.7695 \mathrm{le} 4$ & 19.1190 \\
\hline 5 & 15.454 & $\mathrm{MM} R$ & 0.4349 & 2.98397 e 6 & $1.14343 \mathrm{e} 5$ & 71.6634 \\
\hline
\end{tabular}

Totals : $4.16387 \mathrm{e} 6$ 1.6848le5

*末 End of Report *
* 
Data File E: \DATA $\backslash$ TXF $\backslash$ UGZ-1 $\backslash 7 . D$

Sample Name: $2 \#$
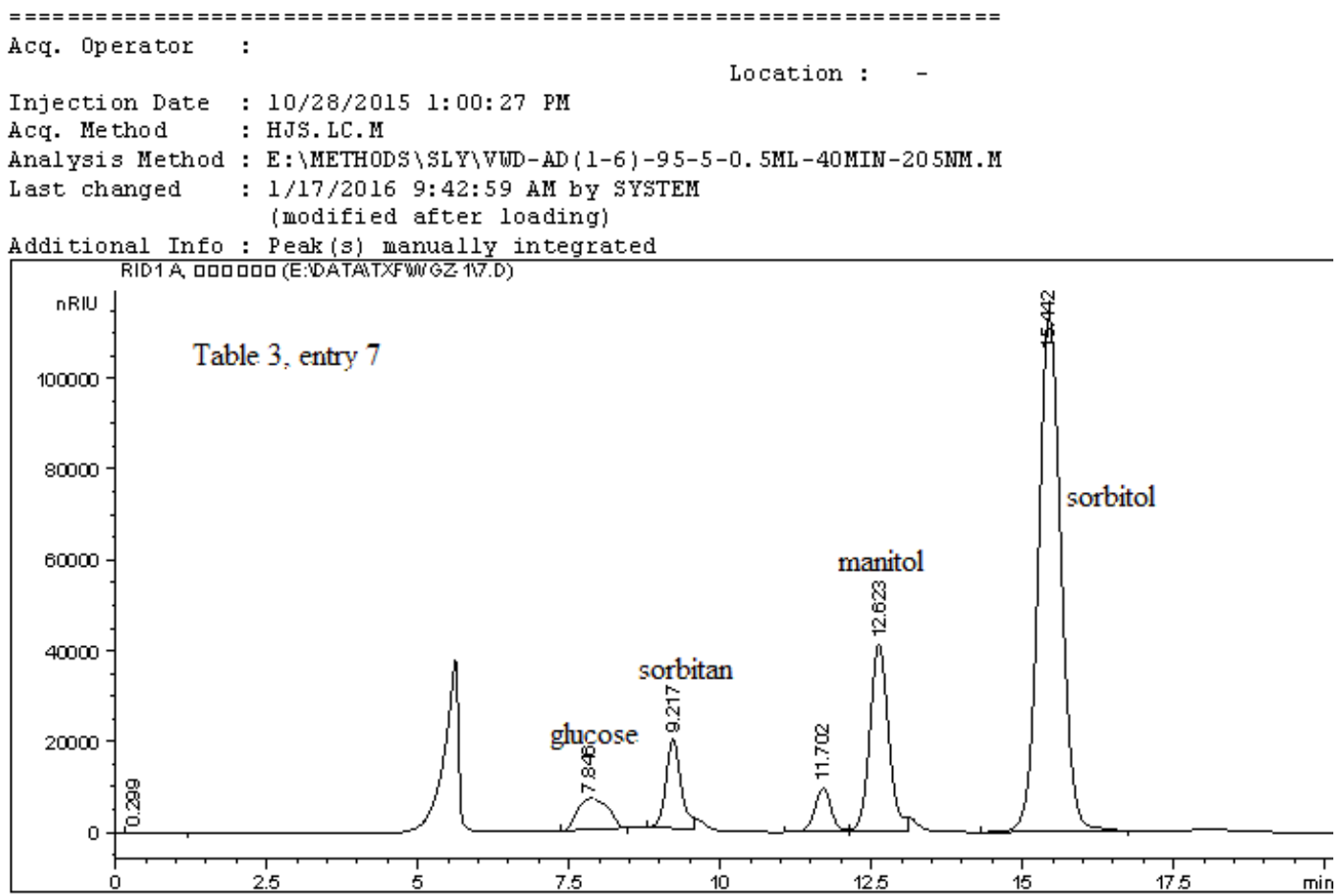

Area Percent Report

\begin{tabular}{|c|c|c|}
\hline Sorted By & signal & \\
\hline Multiplier & 1. 0000 & \\
\hline Dilution & 1.0000 & \\
\hline Sample Amount: & $: \quad 20.00000$ & (not used in calc.) \\
\hline
\end{tabular}

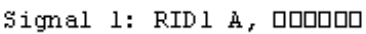

\begin{tabular}{|c|c|c|c|c|c|c|}
\hline $\begin{array}{c}\text { Peak } \\
\#\end{array}$ & $\begin{array}{c}\text { Re tTime } \\
\text { [min] }\end{array}$ & Type & $\begin{array}{l}\text { Width } \\
\text { [min] }\end{array}$ & $\begin{array}{c}\text { Area } \\
{\left[\mathrm{nRIU}^{*} \mathrm{~s}\right]}\end{array}$ & $\begin{array}{l}\text { Height } \\
\text { [nRIU] }\end{array}$ & Area \\
\hline 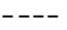 & -- & & ---- & |-------- & |-------- & $|-------|$ \\
\hline 1 & 0.299 & BB & 0.5097 & 251.36784 & 5.82747 & $5.445 e-3$ \\
\hline 2 & 7.846 & $\mathrm{MM} R$ & 0.5778 & 2. $36648 \mathrm{e} 5$ & 6826.17969 & 5.1261 \\
\hline 3 & 9.217 & $M F R$ & 0.2857 & $3.37039 \mathrm{e} 5$ & $1.96612 \mathrm{e} 4$ & 7.3007 \\
\hline 4 & 11.702 & $M F R$ & 0.3153 & 1.81820 e 5 & 9611.19727 & 3.9384 \\
\hline 5 & 12.623 & FM $R$ & 0.3617 & 8.93704 e 5 & $4.11767 \mathrm{e} 4$ & 19.3586 \\
\hline 6 & 15.442 & $\mathrm{MM} \mathrm{R}$ & 0.4360 & $2.96710 \mathrm{e} 6$ & $1.13430 \mathrm{e} 5$ & 64.2707 \\
\hline
\end{tabular}

Totals : 4.61656e6 $1.90711 \mathrm{e} 5$

*** End of Report ** 
Data File E: \DATA \TXF\UGZ-1\8.D

Sample Name: $9 \#$
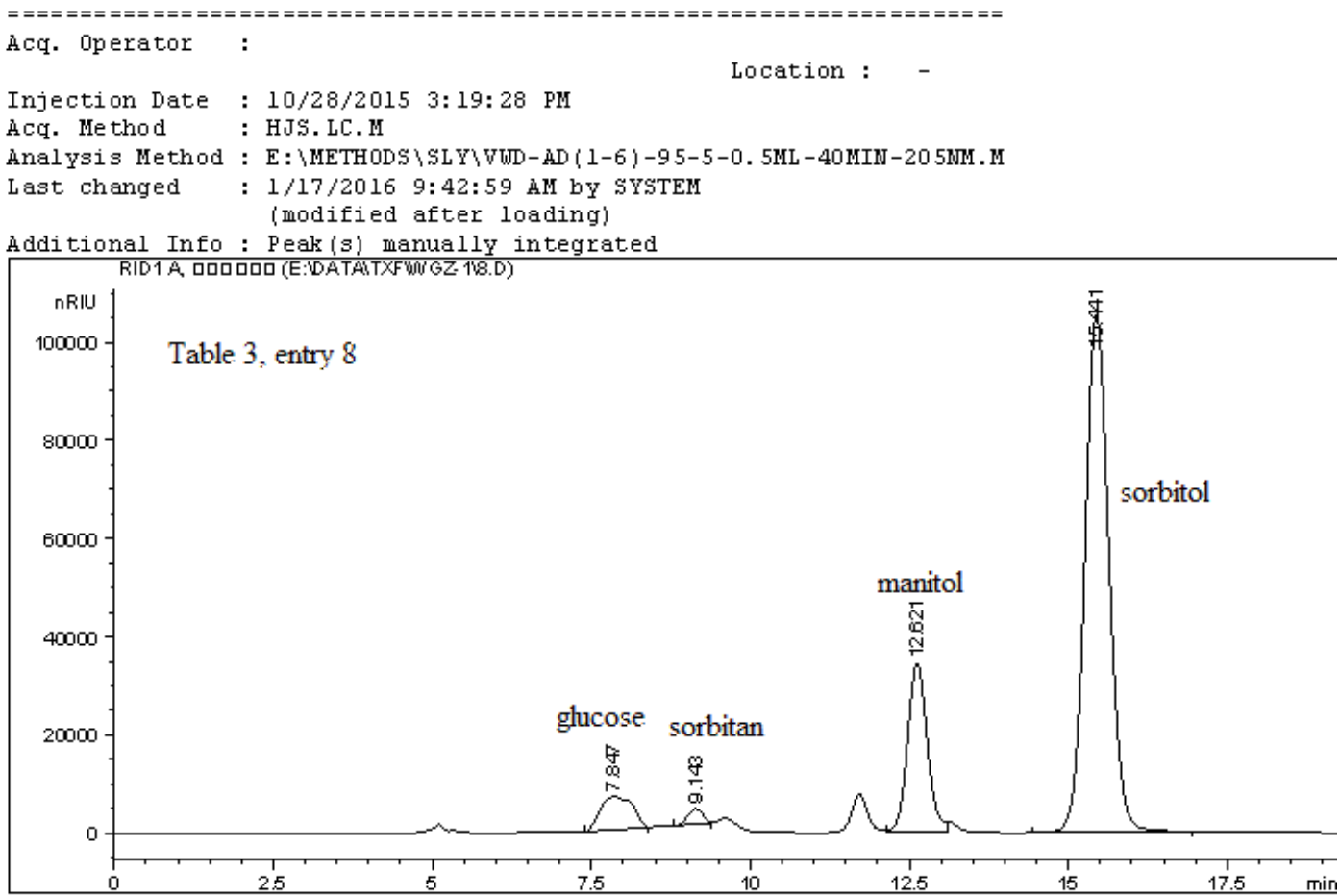

Area Percent Report

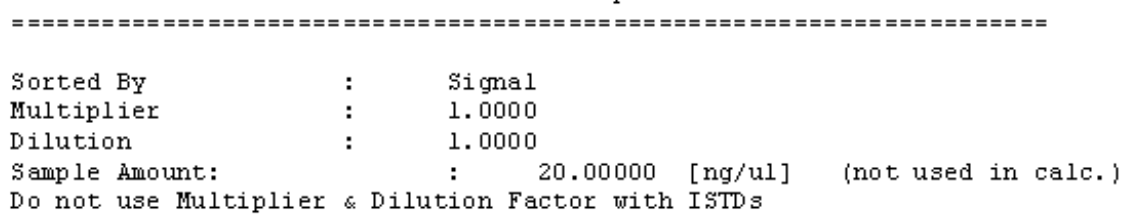

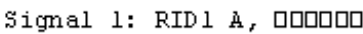

\begin{tabular}{|c|c|c|c|c|c|c|}
\hline $\begin{array}{c}\text { Peak } \\
\#\end{array}$ & $\begin{array}{c}\text { Re tTime } \\
\text { [ min] }\end{array}$ & Type & $\begin{array}{l}\text { Width } \\
\text { [min] }\end{array}$ & $\begin{array}{c}\text { Area } \\
{\left[\mathrm{nRIU}^{*} \mathrm{~s}\right]}\end{array}$ & $\begin{array}{l}\text { Height } \\
\text { [nRIU] }\end{array}$ & Area \\
\hline & - & & 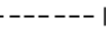 & ---- & ------ & 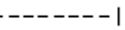 \\
\hline 1 & 7.847 & $\mathrm{MM} R$ & 0.5871 & 2.36708 e 5 & 6719.28809 & 6.2420 \\
\hline 2 & 9.143 & $\mathrm{MM} R$ & 0.2734 & $5.02266 \mathrm{e} 4$ & 3062.11035 & 1. 3245 \\
\hline 3 & 12.621 & $M F R$ & 0.3571 & 7.33417 e 5 & $3.42262 \mathrm{e} 4$ & 19.3401 \\
\hline 4 & 15.441 & $\mathrm{MM} R$ & 0.4382 & $2.77185 \mathrm{e} 6$ & $1.05432 \mathrm{e} 5$ & 73.0934 \\
\hline & 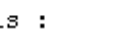 & & & 20 e 6 & 9 e 5 & \\
\hline
\end{tabular}

\section{*ネ* End of Report **}


Data File E: \DATA $\backslash T X F \backslash W G Z-1 \backslash 9 . D$

Sample Name: 10\#
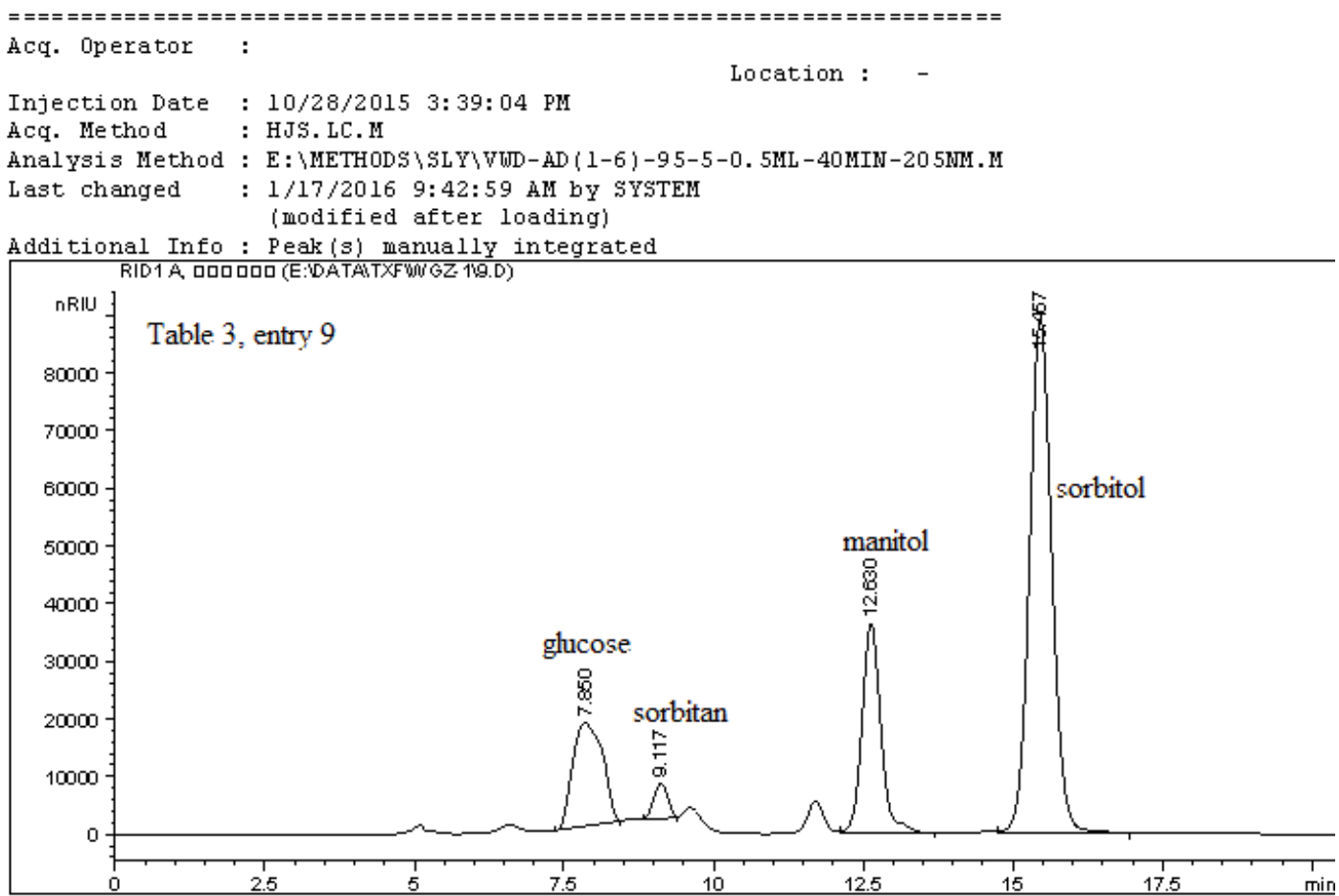

Area Percent Report

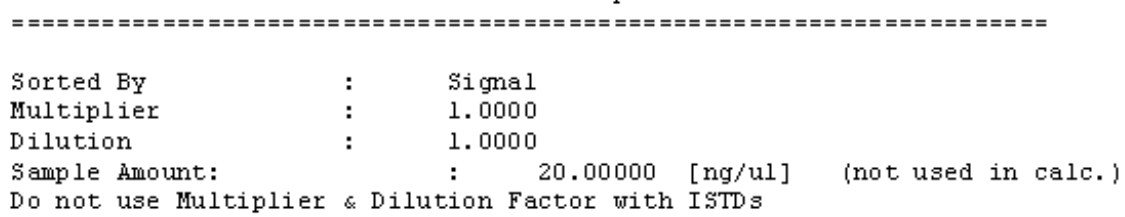

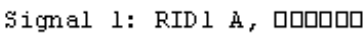

\begin{tabular}{|c|c|c|c|c|c|c|}
\hline $\begin{array}{c}\text { Peak } \\
\#\end{array}$ & $\begin{array}{c}\text { Re tTime } \\
\text { [ min] }\end{array}$ & Type & $\begin{array}{l}\text { Width } \\
\text { [min] }\end{array}$ & $\begin{array}{c}\text { Area } \\
{\left[\mathrm{nRIU}^{*} \mathrm{~s}\right]}\end{array}$ & $\begin{array}{l}\text { Height } \\
\text { [nRIU] }\end{array}$ & $\begin{array}{c}\text { Area } \\
\stackrel{4}{*}\end{array}$ \\
\hline & 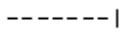 & & & ------ & ------ & . \\
\hline 1 & 7.850 & $\mathrm{MM} R$ & 0.5751 & 6.1866 le 5 & $1.79282 \mathrm{e} 4$ & 16.1355 \\
\hline 2 & 9.117 & $\mathrm{MM} R$ & 0.2753 & 9. 91987 e 4 & 6005.91211 & 2.5872 \\
\hline 3 & 12.630 & FM R & 0.3658 & $7.96962 \mathrm{e} 5$ & $3.63124 \mathrm{e} 4$ & 20.7858 \\
\hline 4 & 15.457 & $\mathrm{MM} R$ & 0.4328 & $2.31934 \mathrm{e} 6$ & $8.93236 \mathrm{e} 4$ & 60.4914 \\
\hline & 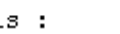 & & & 16 e 6 & 0es 5 & \\
\hline
\end{tabular}

\section{*ネ* End of Report **}

